NIST Technical Note 2041

\title{
Toward a Process to Quantify the Hazard of Fire Protection Design Alternatives
}

\author{
Paul A. Reneke \\ Morgan C. Bruns \\ Stanley W. Gilbert \\ Chandler MacLaren \\ Richard D. Peacock \\ Thomas G. Cleary \\ David T. Butry
}

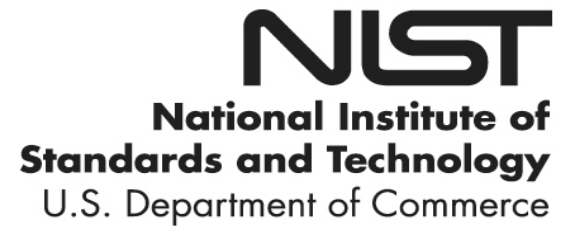




\section{Toward a Process to Quantify the Hazard of Fire Protection Design Alternatives}

\author{
Paul A. Reneke \\ Chandler MacLaren \\ Richard D. Peacock \\ Thomas G. Cleary \\ Fire Research Division \\ Engineering Laboratory
}

\author{
Morgan C. Bruns \\ Virginia Military Institute \\ Stanley W. Gilbert \\ David T. Butry \\ Applied Economics Office \\ Engineering Laboratory
}

This publication is available free of charge from:

https://doi.org/10.6028/NIST.TN.2041

May 2019

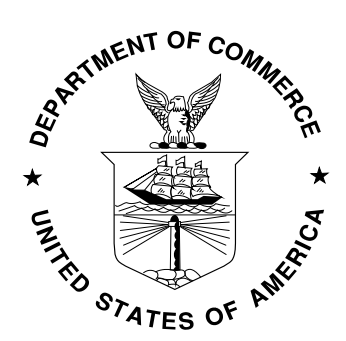

U.S. Department of Commerce Wilbur L. Ross, Jr., Secretary

National Institute of Standards and Technology Walter Copan, NIST Director and Under Secretary of Commerce for Standards and Technology 
Certain commercial entities, equipment, or materials may be identified in this document in order to describe an experimental procedure or concept adequately. Such identification is not intended to imply recommendation or endorsement by the National Institute of Standards and Technology, nor is it intended to imply that the entities, materials, or equipment are necessarily the best available for the purpose.

National Institute of Standards and Technology Technical Note 2041

Natl. Inst. Stand. Technol. Tech. Note 2041, 102 pages (May 2019) CODEN: NTNOEF

This publication is available free of charge from:

https://doi.org/10.6028/NIST.TN.2041 


\begin{abstract}
There are a variety of new, improved, and/or innovative fire protection technologies that have the potential to improve fire protection and life safety in residences including barrier fabrics for upholstered furniture, new nano-scaled flame retardants, and better fire detection technology. However, there is no accepted methodology available to determine the absolute or even relative performance of different technologies. The Engineering Laboratory of the National Institute for Standards and Technology (NIST) has undertaken an effort to develop a method to better quantify the relative improvements in fire safety provided by different technologies to evaluate the impact of changes in prescriptive or performance-based requirements.
\end{abstract}

In order to better understand the overall process for the analysis, this report examines the process for quantitative calculation of fire hazard of fire protection design alternatives using as an example case study the potential benefits of alternate requirements for residential smoke alarms. The case study will help define the process, data, and tools necessary for a quantitative analysis of changes in fire protection designs. The long-term goal of the project is to develop a well-defined process and set of tools for such analyses.

This report shows the result of the case study as well as a discussion of the procedure itself. Several conclusions are apparent. With the speed of current machines and zone fire models, it is practical to generate tens of thousands of cases to analyze. There is also a need for additional data, including but not limited to fire data, information about the distribution of floor plans and about type and arrangement of the contents. Finally, there needs to be guidance and modeling available to account for the impact of occupants' actions and their interactions with fires.

Going forward, research should focus on a two-prong approach. First, tools are needed to make Monte Carlo analysis more efficient. Second, additional opportunities to apply Monte Carlo analysis to real research problems would develop a base of expertise to further the use of this analysis.

\title{
Key words
}

Assessing new technologies; fire modeling; Monte Carlo; residential egress; residential fire safety; smoke alarms: technology performance metrics 


\section{Table of Contents}

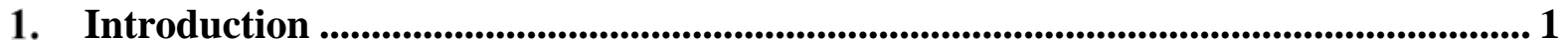

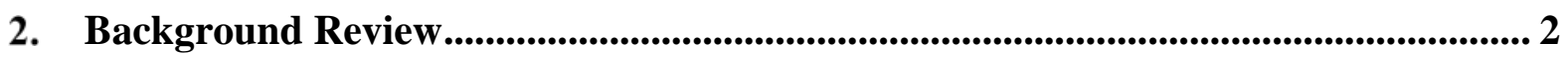

2.1. Fire Hazard Analyses ............................................................................. 2

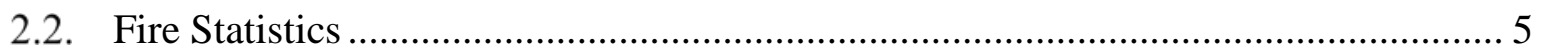

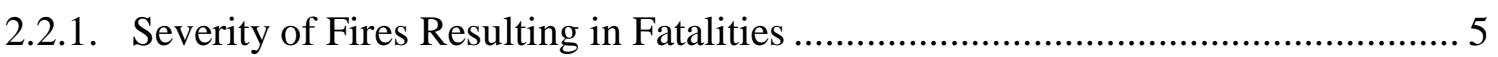

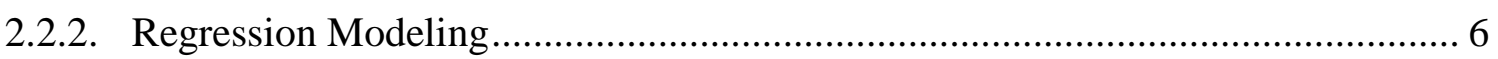

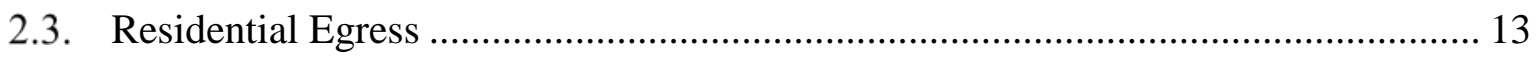

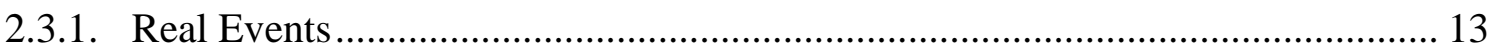

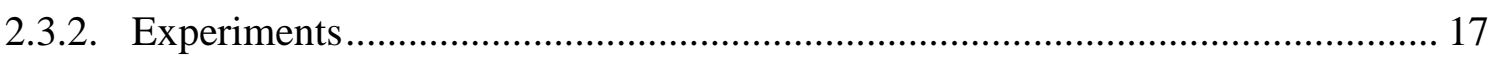

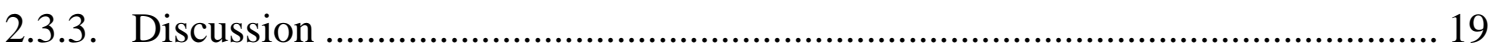

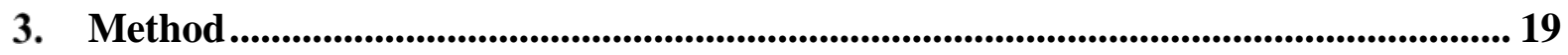

3.1. Community / Building / Occupant Characteristics.................................................... 19

3.1.1. Distribution of Houses.................................................................................... 20

3.1.2. Determining the Floorplan ............................................................................. 21

3.2. Modeling Smoke Detectors ............................................................................ 22

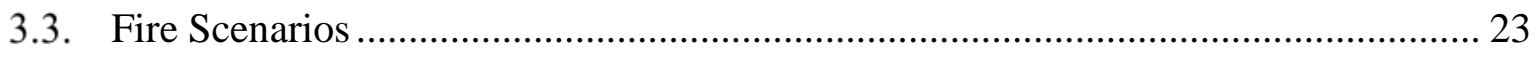

3.4. Monte Carlo Modeling with CFAST .................................................................... 27

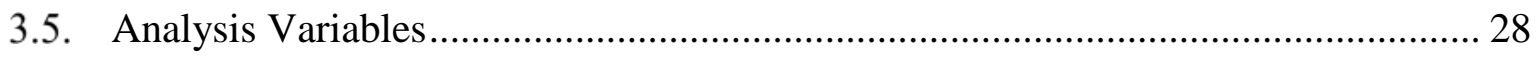

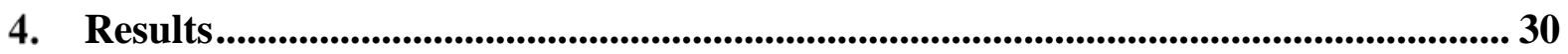

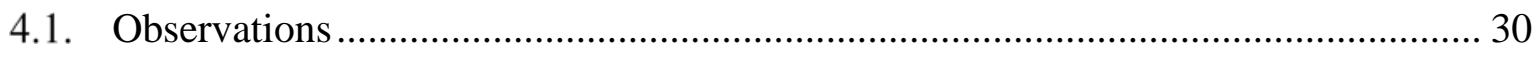

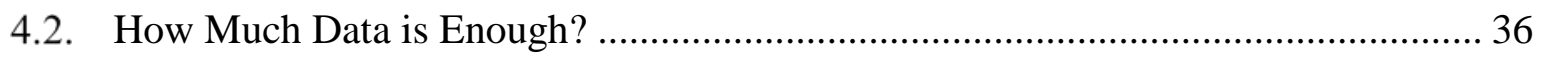

4.3. Smoke Alarm Activation ..................................................................................... 38

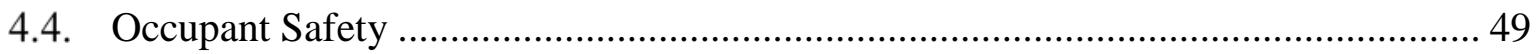

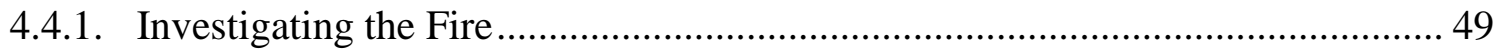

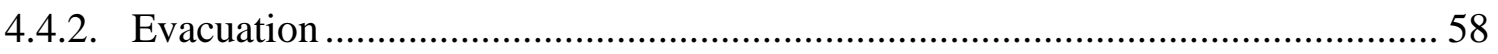

4.4.3. Sensitivity to Estimates of New Alarm Performance............................................. 65

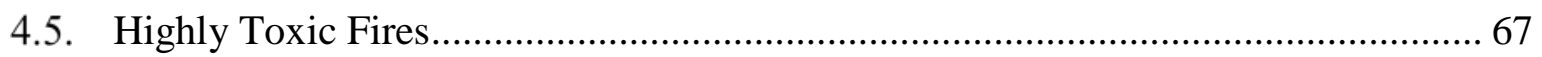

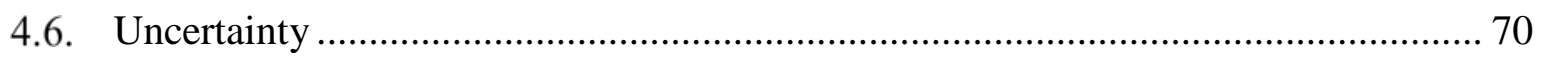

4.6.1. Uncertainty for Quantities of Interest in Model Results .................................... 71

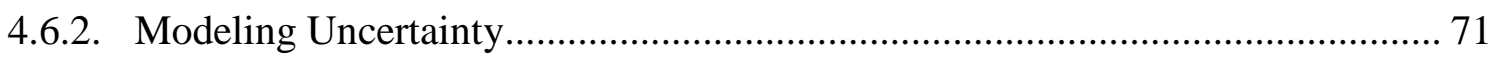

4.6.3. Identifying Potential Issues with Uncertainty ..................................................... 72

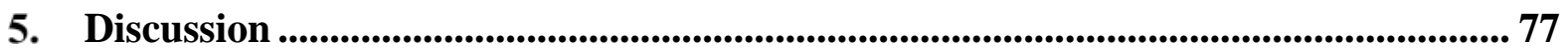

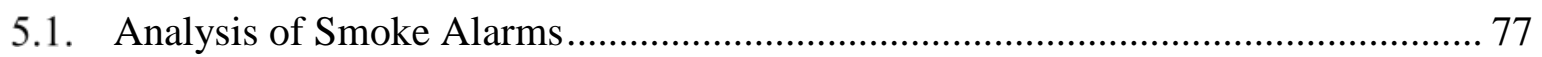




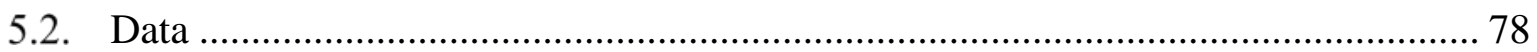

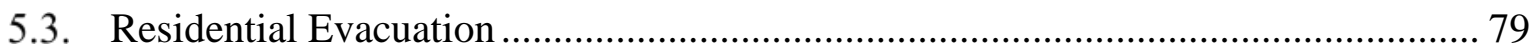

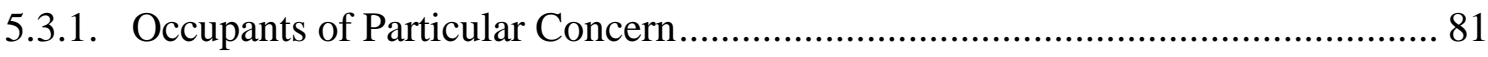

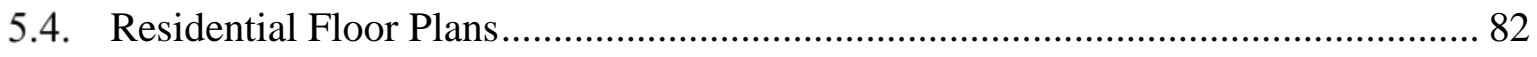

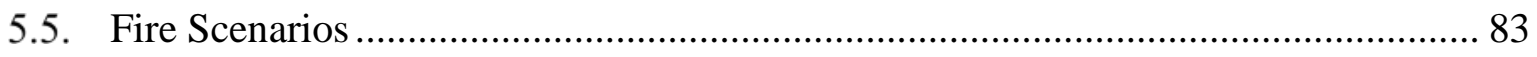

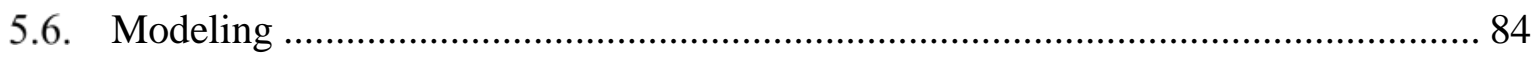

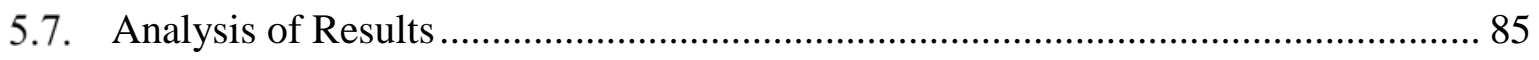

5.8. Uncertainty and Sensitivity in Predictions and Results........................................... 86

6. Conclusions and Future Research Needs ............................................................................ 87

Acknowledgments ....................................................................................................................... 88

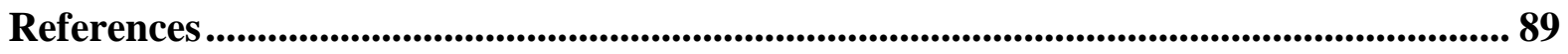

Appendix A. Results of the Deadliness of Fires Regression Model .................................. A-1

\section{List of Tables}

Table 1 Parameters for smoke alarm statistical models........................................................... 23

Table 2. Ignition delay and growth rate of upholstered furniture fires from reference [58]... 24 Table 3 Peak HRR and time to peak HRR for flaming ignition of three mattress types both

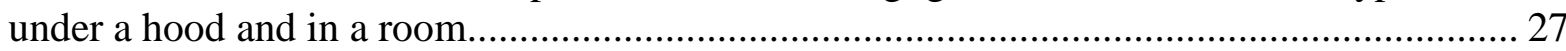

Table 4 Basic statistics for new alarm activation times minus ionization alarm activation times for flaming fires. Uncertainty is shown as a single sample standard deviation. ........... 39 Table 5 Basic statistics for new alarm activation times minus photoelectric alarm activation times for flaming fires. Uncertainty is shown as a single sample standard deviation. ........... 42 Table 6 Slope and intercept for differences in activation times for different types of alarms.46 Table 7 Coefficient of variation by fire type showing how much variance is explained by $u$

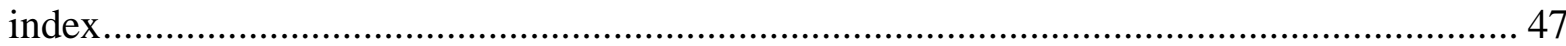

Table 8. Percentage of flaming chair cases where an alarm activation allowed the given time

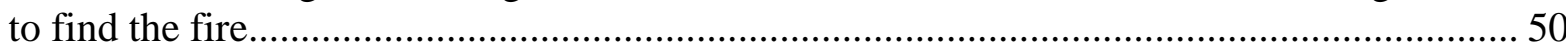
Table 9. Percentage of smoldering chair cases where an alarm activation allowed the given

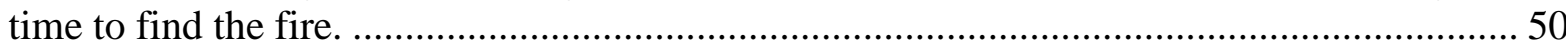
Table 10. Percentage of old mattress cases where an alarm activation allowed the given time to find the fire................................................................................................................... 51 Table 11. Percentage of new mattress cases where an alarm activation allowed the given time

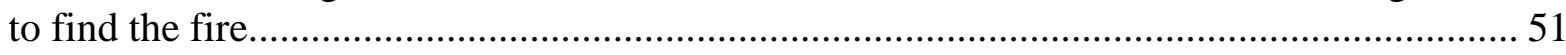
Table 12. Percentage of "flaming fire" cases where an alarm allowed the given time to find

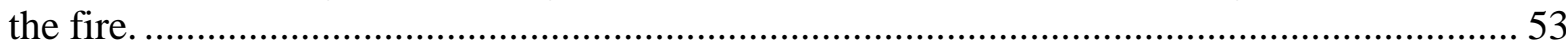
Table 13 Three distributions of the faction of people who find the fire in a particular amount of time 55 Table 14 Cases with Successful Egress for the flaming chair fire with a target FED $=0.3,0.5$, and 1.0 and not accounting for smoke .............................................................................. 59 Table 15 Cases with Successful Egress for the smoldering chair fire with a target FED $=0.3$ and not accounting for smoke. 
Table 16. Summary of CFAST bias and uncertainty for selected predicted results.............. 72

Table 17. Number of test cases run for different categories of scenarios............................ 74

Table 18. Number of cases where untenable conditions were calculated before alarm activation.

Table 19. Regression analysis of analysis predictors with ASET for flaming and smoldering fires, when the fire has a finite ASET (when fire room is connected to alarm room)........... 76

\section{List of Figures}

Figure 1 Relative impact of the day of the week on the deadliness of the fire. Uncertainty bars represent $95 \%$ confidence limits.

Figure 2 Relative impact of the time of day on the deadliness of the fire. Uncertainty bars represent $95 \%$ confidence limits.

Figure 3 Relative impact of the number of floors on the deadliness of the fire. Uncertainty bars represent $95 \%$ confidence limits.

Figure 4 Relative impact of the area of fire origin on the deadliness of the fire.

Listed in order of most deadly to least. Uncertainty bars represent $95 \%$ confidence limits.. 10

Figure 5 Relative impact of the heat source for the fire on its deadliness.

Listed in order of most deadly to least. Uncertainty bars represent 95 \% confidence limits.. 10

Figure 6 Relative impact of the first item ignited on the deadliness of the fire.

Items listed from most deadly to least. Uncertainty bars represent $95 \%$ confidence limits. . 11

Figure 7 Relative impact of the item contributing most to flame spread on the deadliness of

the fire. Items listed in order of most deadly to least. Uncertainty bars represent $95 \%$

confidence limits.

Figure 8 Relative impact of the cause of ignition on the deadliness of the fire.

Causes listed in order from most deadly to least. Uncertainty bars represent $95 \%$

confidence limits.

Figure 9 Cumulative Distribution Function (CDF) for the total area of the home............... 21

Figure 10 Distribution of linear growth time for flaming chair....................................... 25

Figure 11 Triangular distribution for linear growth time for flaming chair ......................... 26

Figure 12 Histogram of percentage for each fire type. .................................................. 31

Figure 13 Histogram of percentage of failed cases by fire type. ....................................... 32

Figure 14 Histogram of percentage of cases with $n$ doors between the fire and smoke

alarm.

Figure 15 Histogram of percentage of cases with n doors between fire room and smoke alarm in cases with a path from fire to the alarm.

Figure 16 Histogram of percentage of cases with each fire type including all cases and only cases where the fire room is connected to the room containing a smoke alarm.

Figure 17 Histogram of percentage of cases with $n$ rooms for all cases

Figure 18 Percentage of cases with $n$ rooms where fire is not connected to a room with a smoke alarm. 35

Figure 19 Comparison of the percentage of cases with $n$ rooms for all cases and only cases where fire is connected to a room with a smoke alarm.

Figure 20 Bin values for histogram of the calculated detection time differences between

ionization and new alarms for new mattress fires. 
Figure 21 Showing the absolute value of the coefficient of variation versus number of bootstrap runs for values calculated for the flaming chair fires........................................ 38 Figure 22 Distribution of differences in new minus ionization alarm activation time for flaming fires.

Figure 23 Close up of distribution of differences in new minus ionization alarm activation time for flaming fires.

Figure 24 Distribution of difference in new and ionization alarm activation time for smoldering chair.

Figure 25 Distribution of differences in new minus photoelectric alarm activation time for flaming fires.

Figure 26 Distribution of differences in new minus photoelectric alarm activation time for smoldering fires.

Figure 27 For flaming chair, difference and uncertainty in activation times based on average activation time.

Figure 28 For smoldering chair, difference and uncertainty in activation times based on average activation time. 48

Figure 29 Linear response of the 4 alarms to time required to investigate a fire upon detection. 52

Figure 30 Percent of ionization alarm activation for different times to find fire and 11 combinations of smoldering and flaming fires 54 Figure 31 Performance of successfully finding the fire for 4 alarms across all distributions of flaming and smoldering fires for the fast distribution of finding fires 56 Figure 32 Performance of successfully finding the fire for 4 alarms across all distributions of flaming and smoldering fires for the medium distribution of finding fires. 57 Figure 33 Performance of successfully finding the fire for 4 alarms across all distributions of flaming and smoldering fires for the slow distribution of finding fires.............................. 58 Figure 34 For flaming chair fire, percentage of cases with successful egress for FED $=0.3$ and not accounting for smoke. 60 Figure 35 For smoldering chair fire, percentage of cases with successful egress for FED $=0.3$ not including the impact of smoke tenability. 61 Figure 36 Overall performance of 4 alarms over range of smoldering and flaming fires, FED $=0.3$ and no smoke considered, using the medium speed distribution. 62 Figure 37 For flaming chair fire, percentage of cases with successful egress for FED $=0.3$ and smoke. 63 Figure 38 For smoldering chair fire, percentage of case with successful egress for FED $=0.3$ when including the tenability impact of smoke. 64 Figure 39 Overall performance of 4 alarms over range of smoldering and flaming fires, FED $=0.3$ and smoke considered, using the medium speed distribution.... 65 Figure 40 Overall performance of 4 alarms over range of smoldering and flaming fires, $\mathrm{FED}=0.3$ and smoke considered, using the medium speed distribution and increasing the mean smoke activation value for the new alarm by $20 \%$. Figure 41 Overall performance of 4 alarms over range of smoldering and flaming fires, FED $=0.3$ and smoke considered, using the medium speed distribution and decreasing the mean new smoke alarm activation value for the new alarm by $20 \%$. 67 Figure 42 Overall performance of 4 alarms over range of highly toxic smoldering and flaming fires, FED = 0.3 and smoke considered, using the medium speed distribution. 68 
Figure 43 Overall performance of 4 alarms over a range of highly toxic smoldering and flaming fires, FED = 1.0 and smoke considered, using the medium speed distribution. ....... 69 Figure 44 Percent of successful cases for egress for given time from high toxicity fires with

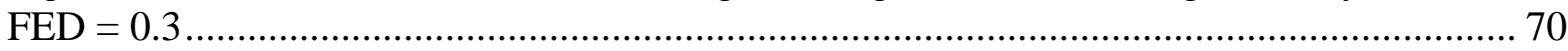

Figure 45. Multilayer model for regression of important analysis variables....................... 73 


\section{Introduction}

In 2015, structure fires injured nearly 15000 people and killed 3400 people in the United States [1]. Of the people who died in fires $77 \%$ or 2630 died in home structure fires, which includes apartment buildings and other multi-family housing [1]. These facts suggest that to impact the civilian death toll requires a focus on home structure fires.

There are a variety of fire protection technologies that have the potential to improve life safety in residences such as barrier fabrics for upholstered furniture, new nano-scaled flame retardants, or better fire detection technology. Currently, there is no accepted methodology to determine the absolute or even relative performance of new or competing technologies. However, as will be reviewed and discussed later, when faced with the need to estimate the impact of certain technologies on the residential fire problem, researchers have come up with very similar approaches. Typically, the approaches use a given fire model or models to run a large number of simulations, setting up the cases with some kind of Monte Carlo procedure, and doing a statistical analysis of the resulting simulations. Fractional Effective Dose (FED) limit or the possibility of flashover have generally been used as performance criteria. There have even been attempts to describe a standard method for doing the analysis, but no standard of analysis has been fully adopted.

There are a number of challenges to this type of analysis which prevent it from more wide adoption. One problem is the issue of data to define a comprehensive set of modeled scenarios, including its availability and the needed assumptions to fill in any gaps in the data. Examples of issues include appropriate floor plans and their distributions, fire scenarios, and distributions of populations. A second problem is how to run the large number of simulations needed to generate good statistics. Even with zone fire models that can be run quickly, the labor required to set up thousands of runs and process the results by hand is prohibitive. A third problem is deciding what output variables to use in the analysis and how resulting statistical analyses relate to what we see in real communities. Some researchers have attempted to predict actual fire deaths; others have looked at the time to hazardous conditions or flashover or even attempted to predict the probability of hazardous conditions occurring. Each method can have its advantages and disadvantages.

The Engineering Laboratory of the National Institute for Standards and Technology (NIST) has undertaken an effort to develop a method to quantify the relative improvements in fire safety that different technologies can make in a community. The overall goal is to provide tools to evaluate the impact of changes in prescriptive or performance-based codes and standards requirements. While the system will be primarily focused on community impact, it ought to scale down for use on individual residential structures.

In order to better understand the process, this report examines a procedure for quantitative calculation of fire hazard of fire protection design alternatives using as an example case study of the potential benefits of new smoke alarm standards, ANSI/UL 2172015 [2]. The case study will help define the process, data, and tools necessary for a quantitative analysis of changes in fire protection designs. The long-term goal of the project is to develop a welldefined and generally accepted process and set of tools for such analyses. The report is organized as follows: 
- Chapter 2 reviews the available literature on fire hazard analysis, residential evacuation, and fire statistics to inform necessary data, calculations, and analysis required for the case study and for defining the steps of a quantitative analysis procedure.

- Chapter 3 describes the process and choices for input data and calculations with details for the case study.

- Chapter 4 presents the results of the calculations for the chosen analysis variables.

- Chapter 5 discusses the implications of the analysis on the process of fire hazard analysis and provides an assessment of its current limitations.

- Chapter 6 summarizes future research needs to better address quantitative fire hazard analyses.

\section{Background Review}

In its simplest form, the analysis of fire safety during a building evacuation can be described in the concept of ASET/RSET. ASET, the available safe egress time, is the amount of time that conditions in the building allow occupants to safely leave the structure. RSET, the required safe egress time, is the amount of time needed to safely evacuate all the occupants of a building. The basic idea is that ASET should always be greater than RSET in a building to ensure the fire safety design of a particular building [3].

This section reviews the quantitative analysis of fire safety and includes three parts: the calculation of ASET/RSET in fire safety analyses to date (which informs how well we know how to calculate ASET and RSET in residences), the current state of the literature related to residential evacuation (including significant limitations on the use of RSET in residences), and fire statistics including causes and impacts of fires in residences (which informs important fire scenarios and populations that must be considered in analyses).

The review is intended to give an overview of the different aspects of residential egress.

\subsection{Fire Hazard Analyses}

This section provides a brief review of efforts to quantify the impact of different fire safety technologies. The idea of using a statistical approach to understand the impacts of different technologies and/or scenarios is not a new idea. Available engineering calculations and fire models make this kind of analysis relatively straightforward to do. However, researchers have had to create their own ad-hoc method including developing their own critical measurements and criteria for evaluating the results.

Bukowski [4] used a zone model to predict the hazard of various residential upholstered furniture (RUF) fires in a three room layout. The varied parameters were floor plan geometry, wall materials, heat of combustion of the fuel, the smoke yield, the RUF burning rate, and the presence or absence of an open door. These parameters were typically varied to one or two values other than nominal values. Hazard was quantified in terms of gas 
temperatures, hot gas layer (HGL) height, optical density, and thermal FED. It was found that the hazard criteria are most sensitive to the burning rate of the fuel. The results of this analysis are qualitative due to the limited number of cases considered and the fact that these cases are not rigorously connected to real fire scenarios through well-characterized building data.

The methodology described in the preceding paragraph was implemented in the software HAZARD I [5]. HAZARD I was designed with a focus towards single-family residential structures. The zone model FAST in conjunction with evacuation models was used to predict fire losses. The authors cautioned that the results should only be used for comparisons between products as the models were not developed enough to make precise predictions. The HAZARD I model was applied with examples for several residential fire scenarios.

An approach similar to that of the present report was developed by Clarke et al. [6]. The objective of the research was to use fire and egress models in conjunction with fire data to estimate the change in hazard associated with a change in product. Several applications were studied including the hazard of RUF [7]. The relative weightings of the specific fire scenario parameters (e.g., time of day, mobility, house size, etc.) were mostly based on data from the national fire statistics with some weightings for RUF provided by an expert panel. For the furniture application, the modeled fires were assumed to take place in a prototypical ranch home. Fire dynamics and egress were modeled using HAZARD I. Predicted fire deaths compared well with the deaths recorded in the available fire statistics. The research examined the sensitivity of the results to such factors as the locations of the occupants, the potential for occupant rescue, occupant delay in evacuating the house, duration of pre-flaming smoldering, thermal window breakage, and home size.

The hazard of a single room scenario was explored by Babrauskas [8]. HAZARD I was used to predict the hazard for several cases in which the heat release rate (HRR), toxicity, and ignition time of a RUF fire were varied. Hazard was quantified in terms of gas temperature (exceeding $100{ }^{\circ} \mathrm{C}$ ) and toxicity (a time-integrated exposure to the mass concentration of toxic products of combustion exceeding a value of [9] $\left.900\left(\mathrm{~g} / \mathrm{m}^{3}\right) \mathrm{min}^{1}\right)$. It was concluded that life safety is much more strongly dependent on HRR as compared to toxicity. This is primarily a consequence of the fact that only flaming pre-flashover cases were considered in which the toxicity of gases is relatively low.

Peacock et al. [10] studied flashover using several correlations in addition to the Consolidated Model of Fire and Smoke Transport (CFAST) [11]. Flashover is typically defined in terms of the conditions needed to ignite certain target materials within the room of fire origin. Flashover is relevant to hazard in that a post-flashover compartment is certainly untenable and may produce significant toxic gases that may be transported to other rooms within the home. Recommended flashover criteria were taken to be temperatures exceeding $600{ }^{\circ} \mathrm{C}$ and floor heat fluxes greater than $20 \mathrm{~kW} / \mathrm{m}^{2}$. It was found that correlations such as those of Thomas [12] and McCaffrey et al. [13] are able to predict flashover just as well as CFAST for the scenarios considered. In a continuation of this work, Babrauskas et al. [14] found that there was considerable variability in the occurrence of flashover as a function of HRR in rooms of similar geometry. This variability was attributed to differences in the

\footnotetext{
${ }^{1}$ To put this value in context, note that a 1 minute average concentration of $30 \mathrm{~g} / \mathrm{m}^{3}$ would reach lethality after $30 \mathrm{~min}$. The density of air at standard temperature and pressure is $1225 \mathrm{~g} / \mathrm{m}^{3}$ so the toxic material would only have to be $2.4 \%$ mass fraction.
} 
dynamic behavior of HRR versus time curves. Such behavior is not accounted for in typical correlations. CFAST simulations were used to show that there is a broad range of critical HRRs needed for flashover. Although the critical HRR was found to depend strongly on the time at which flashover occurs, simulations indicate that the results are relatively insensitive to the shape of the HRR curve.

In order to assess the reduction in fire losses associated with a changed mattress flammability standard, Ohlemiller and Gann [15] used CFAST to predict the spread of smoke in a four room structure. This structure was similar to that used by Bukowski [4], but with an additional large compartment to account for the rest of the house. Variations were made to the size of the room of fire origin as well as the fire room door opening size. It was found that a reduction in HRR did not eliminate all risk to the occupants, but it did lead to a much reduced probability that a nearby item would be ignited. From an investigation of fire statistics, it was determined that a significant reduction in HRR would result in a significant reduction in the number of flashovers. Consequently, fire losses would be significantly reduced.

The potential for sublethal incapacitation in fires was studied by Peacock et al. [16] using CFAST. In this work, it was noted that a significant limitation of CFAST is an inability to account for the toxicity associated with under-ventilated fires. Three scenarios were simulated: a ranch house, a hotel, and an office. Tenability was accounted for using a thermal Fractional Effective Dose (FED) based on heat and incapacitating asphyxiant gases. Calculation of this FED was based on the models given in ISO 13571 [17]. It was found that time to incapacitation due to heat was much smaller than the time to incapacitation due to asphyxiant gases except for cases of smoldering. Fire deaths due to toxic gas inhalation mostly occur post-flashover.

Several papers have demonstrated methods for propagating uncertainty through fire models. Upadhyay and Ezekoye used the Quadrature Method of Moments (QMOM) to propagate HRR uncertainty through CFAST and an algebraic model for layer height [18]. Layer height cumulative distribution functions (CDFs) were reconstructed using a generalized lambda distribution. The results of the QMOM simulations compared favorably with those obtained by more thorough Monte Carlo simulations. This indicates that efficient methods such as QMOM could be used to adequately propagate uncertainty through fire models.

Monte Carlo simulation of CFAST was used to determine the effects of HRR curve uncertainty on the available safe egress time by Kong et al. [19]. Latin hypercube sampling was used to improve efficiency. Two uncertain parameters, peak HRR and fire growth rate, were considered as random model inputs and modeled as normal or log-normal probability distributions. An extremely large single compartment, representing a commercial building, was considered, and the results were presented along with a sensitivity analysis.

Bruns [20] developed the statistical analysis technique in a mathematically rigorous fashion. The problem being addressed was to predict the impact of different barrier fabrics on the hazards from a fire on the upholstered furniture. The technique made use of test fires to determine the HRR rate of chairs of the same design with two different covering fabrics and six barrier fabrics. A simple empirical correlation by McCaffery, Quintiere, and Harkleroad, the MQH) correlation [21] and CFAST simulations were used to estimate the impact of the 
12 combinations of cover fabrics and barrier fabrics in a three room floorplan. Estimates of the probability of each combination reaching flashover in the living rooms and lethal conditions in the bedroom were made.

Notarianni [22] defined the process for understanding the uncertainty inherent in fire protection modelling applications through a case study on residential fire sprinklers. The work demonstrated the need to include distributions for uncertain inputs and the impact of uncertainty in the selection and analysis of design criteria for fire protection engineering designs. Notarianni and Parry [23] describe steps for performance-based design in fire protection applications that include uncertainty throughout the design process. In part, this report builds on the work of Notarianni to better understand the tools and calculations needed for the statistical treatment of fire modeling in the design process.

\subsection{Fire Statistics}

The National Fire Incident Reporting System (NFIRS) is a reporting system used by fire departments nationwide to report on their activities. The system is maintained by the U.S. Department of Homeland Security and the U.S. Fire Administration and is designed to capture all activities engaged in by a fire department, including fires, emergency medical service (EMS) and community outreach. The system is voluntary at the national level, so some departments do not use the system or report data from it. Additional data and analyses are available from the National Fire Protection Association(www.nfpa.org).

The NFIRS system records the time, date and location of all incidents, the type of the incident (e.g., fire, EMS call, hazardous materials incident, service call, etc.), property use, equipment and personnel on the call, number type and severity of casualties, actions taken, and a host of other data. For fires specifically, NFIRS collects information on the size of the fire, room of origin, heat source, item first ignited, human and other factors contributing to ignition, presence and effectiveness of detection and automatic suppression equipment among other data.

Data for NFIRS is filled out by firefighters at the scene, so the information it contains is typically limited to the information a firefighter at the scene would have. For example, fire deaths are defined as any fire casualties resulting in death within one year. However, it seems likely that follow-up on casualties is sporadic at best. Often data that is not required is left unentered. For example, extent of fire spread is recorded for only about $30 \%$ of fires. Other systematic problems can occur. For example, a number of large departments report in excess of $80 \%$ of the fires they respond to are confined fires (the nationwide average is less than $40 \%$ ). Nevertheless, while NFIRS has known reporting problems, it is still the best data set available for understanding the nature and extent of the urban fire problem in the United States.

\subsubsection{Severity of Fires Resulting in Fatalities}

Data from NFIRS can provide insight into important characteristics of fire scenarios that would be important to capture in a fire hazard analysis. This section identifies the characteristics of fires that contribute to their deadliness. A number of factors contribute to the number of deaths that occur from fire, including the number of fires that occur, number of people exposed to a fire, characteristics of the people who are exposed (e.g., age, frailty [24], 
sex), and characteristics of the environment and fire. This study is interested the characteristics of the environment and fire that contribute to fatal fires. As such it abstracts from the characteristics of people exposed to fire, and from the number of fires and number of people exposed to fires.

In NFIRS, there is no data on the number or characteristics of people exposed to a given fire (although it does have information on people who are injured or killed in fires). Since data on people exposed to fire is not generally available, it is beyond this study to account for it.

Characteristics that are considered are time of day, day of week, number of floors in the building, room of origin of the fire, heat source for the fire, item first ignited, item most contributing to flame spread, and extent of fire spread.

Some $20 \%$ of the data is selected randomly and reserved as a test set, while the remaining $80 \%$ is used to fit the models.

A number of records were removed if data components were missing, if there were obvious miscodings, and when the room of origin was listed as 'workspace'. The latter was removed because there were so few single-family residential fires with a 'workspace' as the room of origin that it detracted from the analysis. Total number of records removed for these reasons was 106 477. Total number of fires in the training set was 673 353. Total number of fires in the test set was 169083.

\subsubsection{Regression Modeling}

Two regression models were run to test the relationship between a number of predictor factors, $X_{i j}$, on the probability of fire fatality, $P\left(\right.$ death $\left._{i}\right)$, a representation of the deadliness of a fire. The first model excluded the NFIRS field fire_sprd, and the second one included it. Both models were logistic regression models evaluated using a generalized linear models (GLM) approach.

The first model is estimated as:

$$
g\left(P\left(\text { deaths }_{i}\right)\right)=\sum_{j=1}^{n} X_{i j} \beta_{j}
$$

where $X_{i j}$ is the value of the $j$ th predictor for the $i$ th fire (note that all the predictors in the estimation are dummy variables), $\beta_{j}$ is the regression coefficient for the $j$ th predictor, and $g$ is the linking function -- in this case the logit function.

The second model is estimated as:

$$
g\left(P\left(\text { deaths }_{i}\right)\right)=\sum_{k=1}^{m} F_{i k} \alpha_{k}+\sum_{j=1}^{n} X_{i} j \beta_{j}
$$

where $F_{i k}$ is the dummy variable for the $k$ th fire size for the ith fire, $\alpha_{k}$ is the regression coefficient for the $k$ th fire size, and all the remaining terms have the same meaning as above.

Complete results are listed in Appendix A. 
To test the accuracy of the models, predictions were generated for the data. Predictions are generated for all the data, but we are primarily interested in the predictions on the test set.

From the predictions we generate root-mean-square errors on the (out-of-sample) test set.

$\begin{array}{ll}\begin{array}{ll}\text { Include Fire } \\ \text { Spread }\end{array} & \begin{array}{l}\text { RMS } \\ \text { Error }\end{array} \\ \text { No } & 0.0902 \\ \text { Yes } & 0.0900\end{array}$

Interestingly, there is little difference between the 2 models. In particular, adding an indicator of the fire size (which is clearly a significant factor in fire deaths) does not substantially decrease the error in the out-of-sample predictions. To the extent that fire size contributes to fire deaths, its effect is fully explained by the fire environment variables.

Since fire spread contributes so little to predictability of the deadliness of fires, it is ignored for the rest of this analysis.

The graphs below show the coefficients, $\beta_{\mathrm{j}}$, from the estimation. Note that the coefficients for each group of dummy variables have been centered in the graphs below. That is possible because the coefficients for each group are only defined up to a constant. Specifically, what is graphed is the value of the estimated coefficient for that variable minus the mean of the coefficients for all the variables in that group.

Figure 1 shows that the day of week has no effect on the dangerousness of fires.

As can be seen in Figure 2, fires that occur in the early morning (when everyone is probably asleep) are significantly deadlier than fires that occur in the afternoon.

Except for homes with zero floors, the number of floors in a house has no effect on the deadliness of a fire as seen in Figure 3 (although an allowed input in NFIRS, it is not clear what it means for a single-family residence to have zero floors).

Figure 4 shows that area of origin has the greatest range of effect of any of the variables tested. Fires that start in service and exterior spaces are significantly less deadly than other fires. The deadliest room for a fire to start in is the living room. It is important to point out that this is a simultaneous regression with the other factors listed. So, for example, fires that start in the kitchen are almost invariable among the least deadly. However, there is a very large overlap between kitchen fires and cooking fires. What this means is that the location is not the significant factor in the relatively low deadliness of kitchen fires.

For the most part, the NFIRS field heat_source does not distinguish between more and less deadly fires as seen in Figure 5. The only exception to this is smoking materials which are significantly deadlier than fires from other heat sources.

Looking at item first ignited in Figure 6, fires that start in bedding, upholstered furniture, and "Soft Goods" (like clothing), are significantly deadlier than fires that start in other materials. Fires that start in structural components are the least deadly. 
For the closely related field of item most contributing to flame spread shown in Figure 7, fires where liquids (excluding cooking materials) contribute most to flame spread are the most dangerous. Fires where cooking or electrical materials contribute most to flame spread are the least dangerous.

It is worth noting that the "Item First Ignited" and "Item Most Contributing to Flame Spread" fields differ only when the item most contributing to flame spread is different from the item first ignited. They differ only in $10.6 \%$ of fires. Comparing the results for the two groups of variables, it seems likely that item contributing most to spread is carrying most of the weight for the two variables. Likely, "Item First Ignited" only matters when the two variables differ.

Finally, in Figure 8, considering cause of ignition, fires under investigation are the deadliest, while fires with natural causes are the least deadly. Interesting, fires with intentional causes are less deadly than fire with unintentional causes. It is not clear how to interpret the results for fires whose cause is under investigation, since it is not an actual cause. It seems likely that some of those fires are under investigation precisely because there were fatalities. It is also not clear how these results would change if the underlying cause were used rather than the "investigating” placeholder.

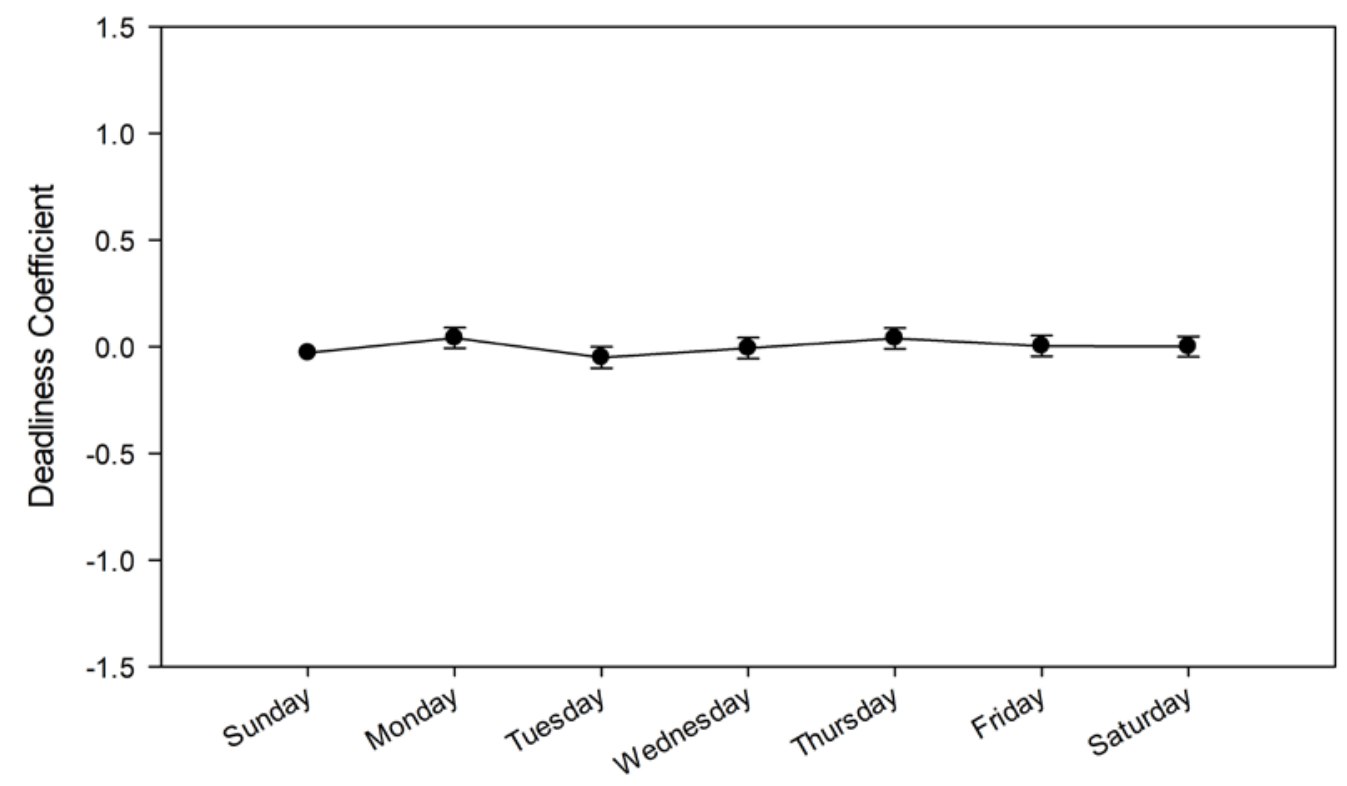

Figure 1 Relative impact of the day of the week on the deadliness of the fire. Uncertainty bars represent $95 \%$ confidence limits. 


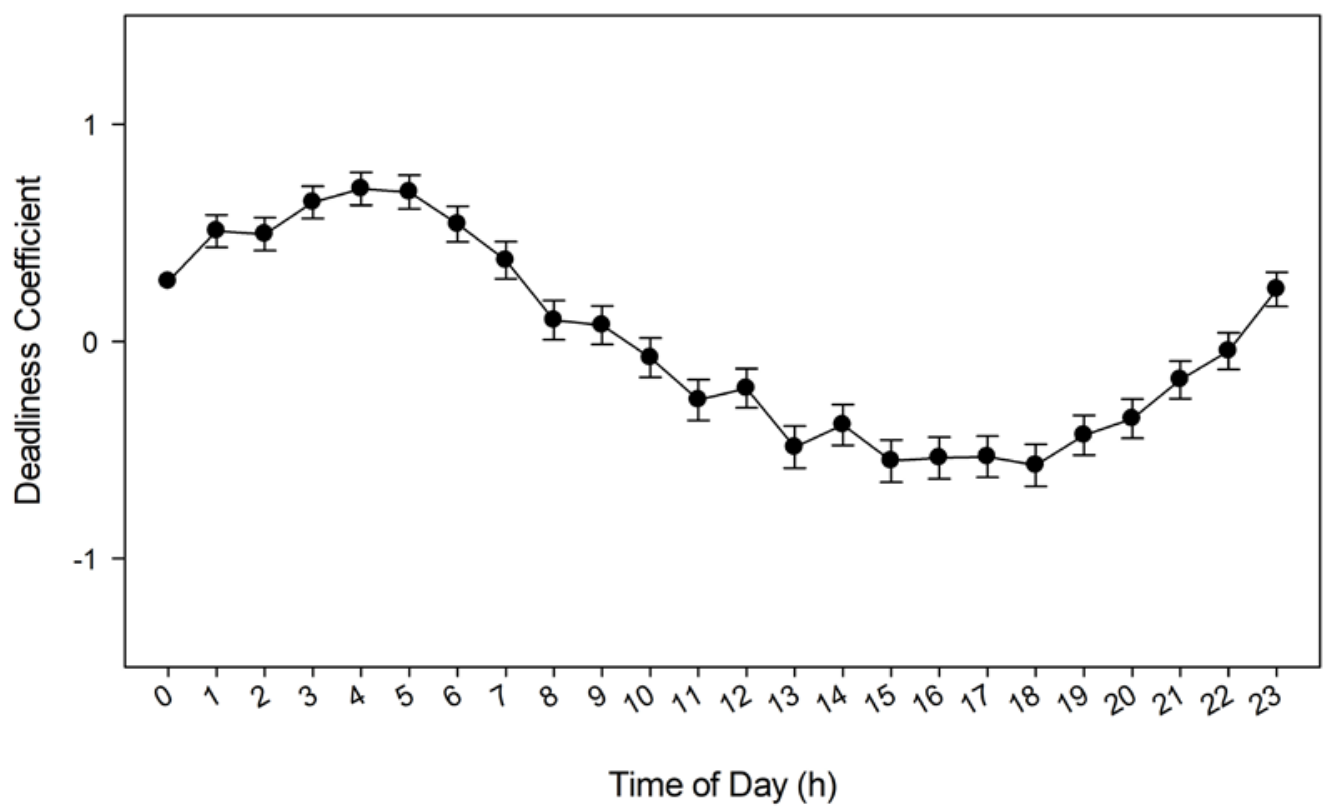

Figure 2 Relative impact of the time of day on the deadliness of the fire. Uncertainty bars represent $95 \%$ confidence limits.

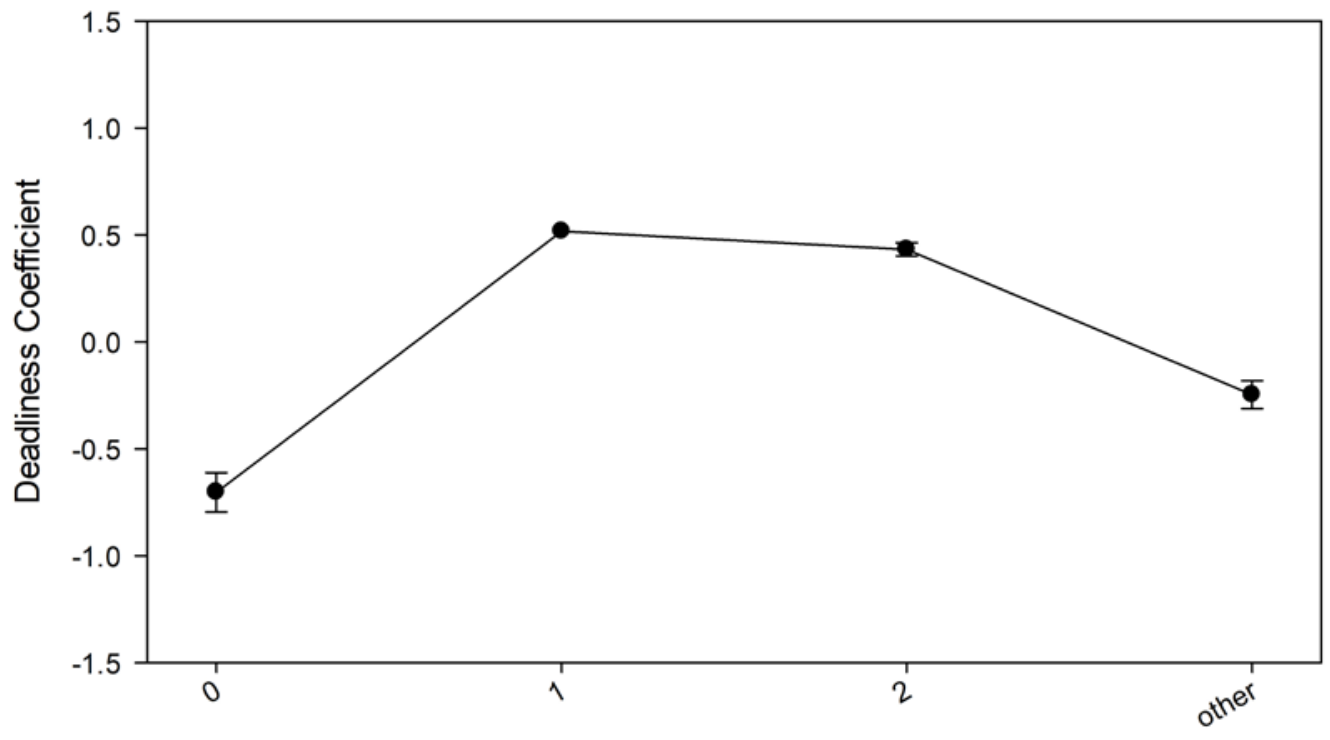

Floor

Figure 3 Relative impact of the number of floors on the deadliness of the fire. Uncertainty bars represent $95 \%$ confidence limits. 


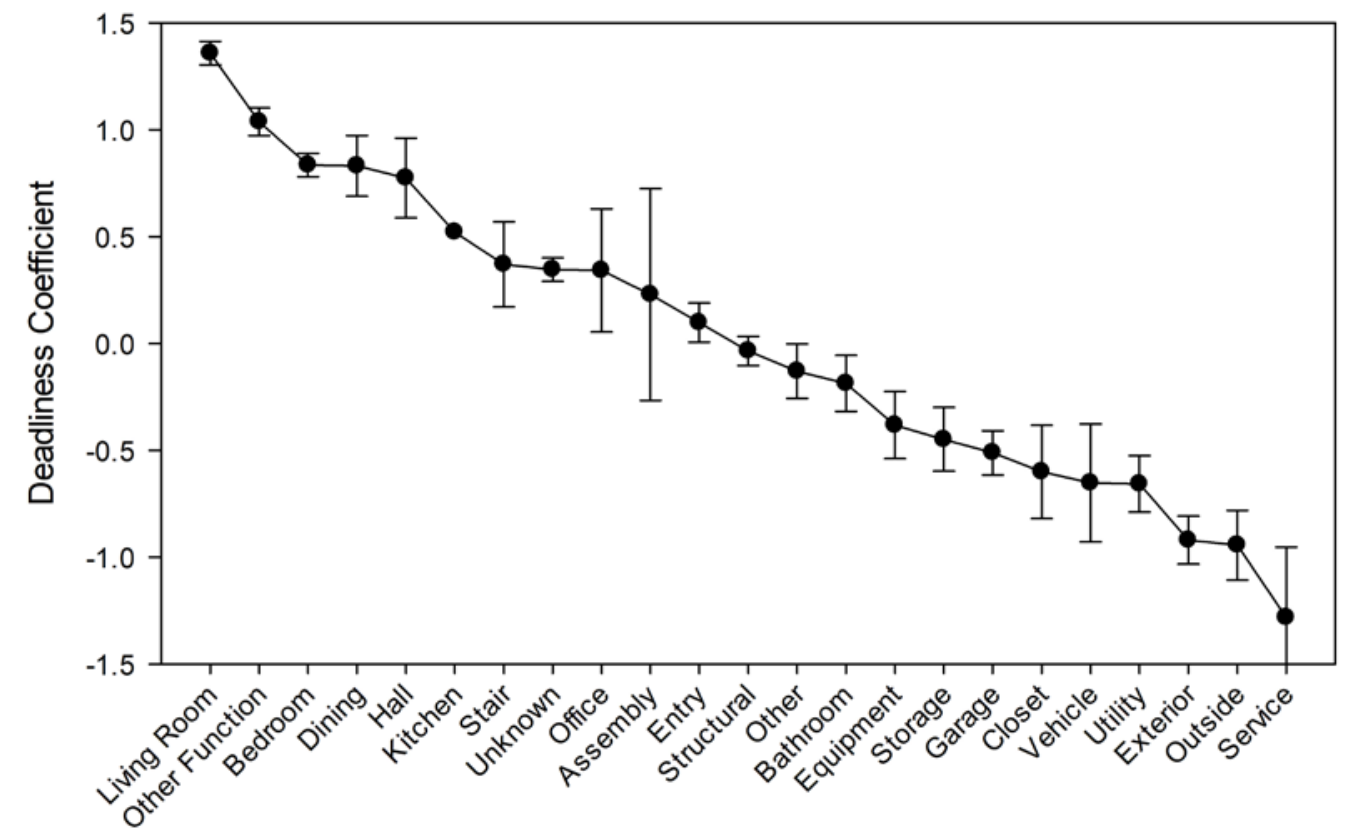

Figure 4 Relative impact of the area of fire origin on the deadliness of the fire. Listed in order of most deadly to least. Uncertainty bars represent $95 \%$ confidence limits.

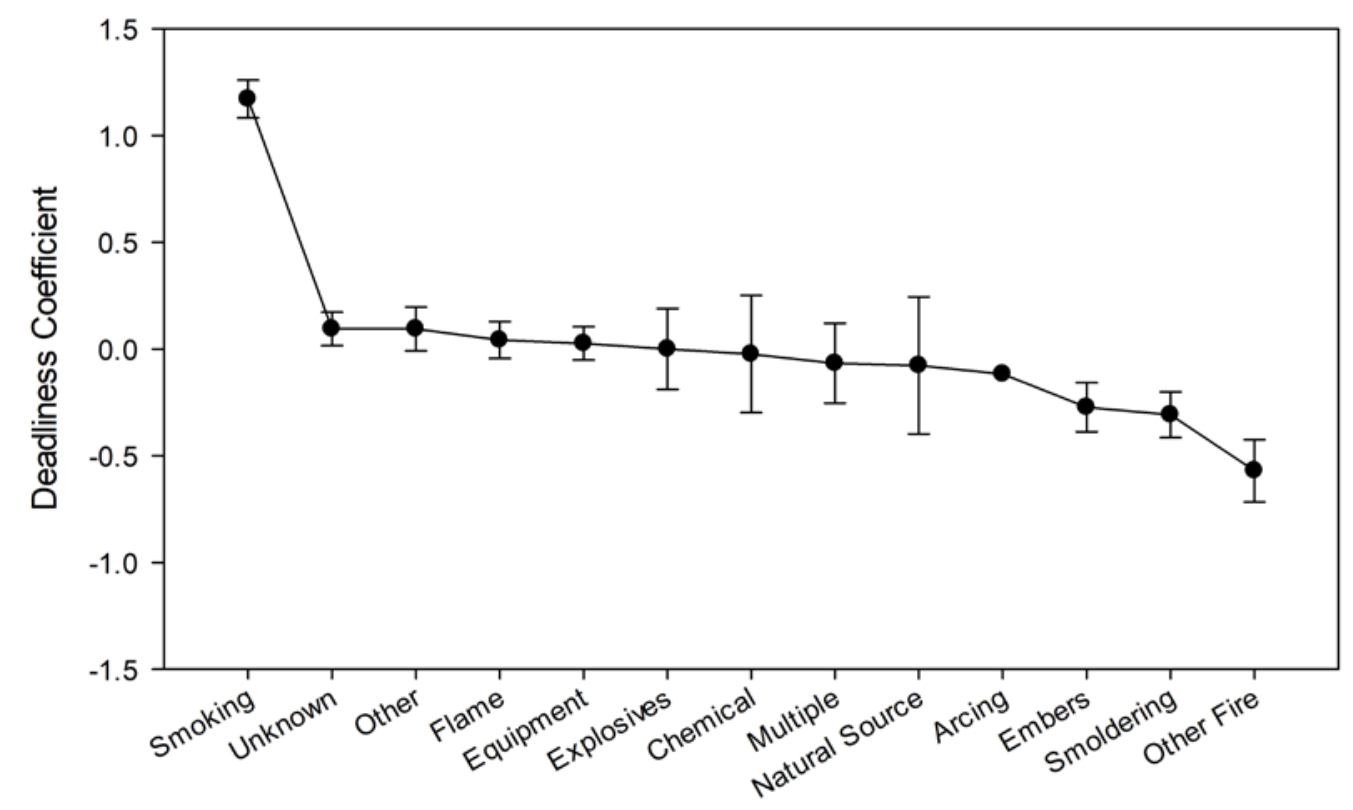

Figure 5 Relative impact of the heat source for the fire on its deadliness. Listed in order of most deadly to least. Uncertainty bars represent $95 \%$ confidence limits. 


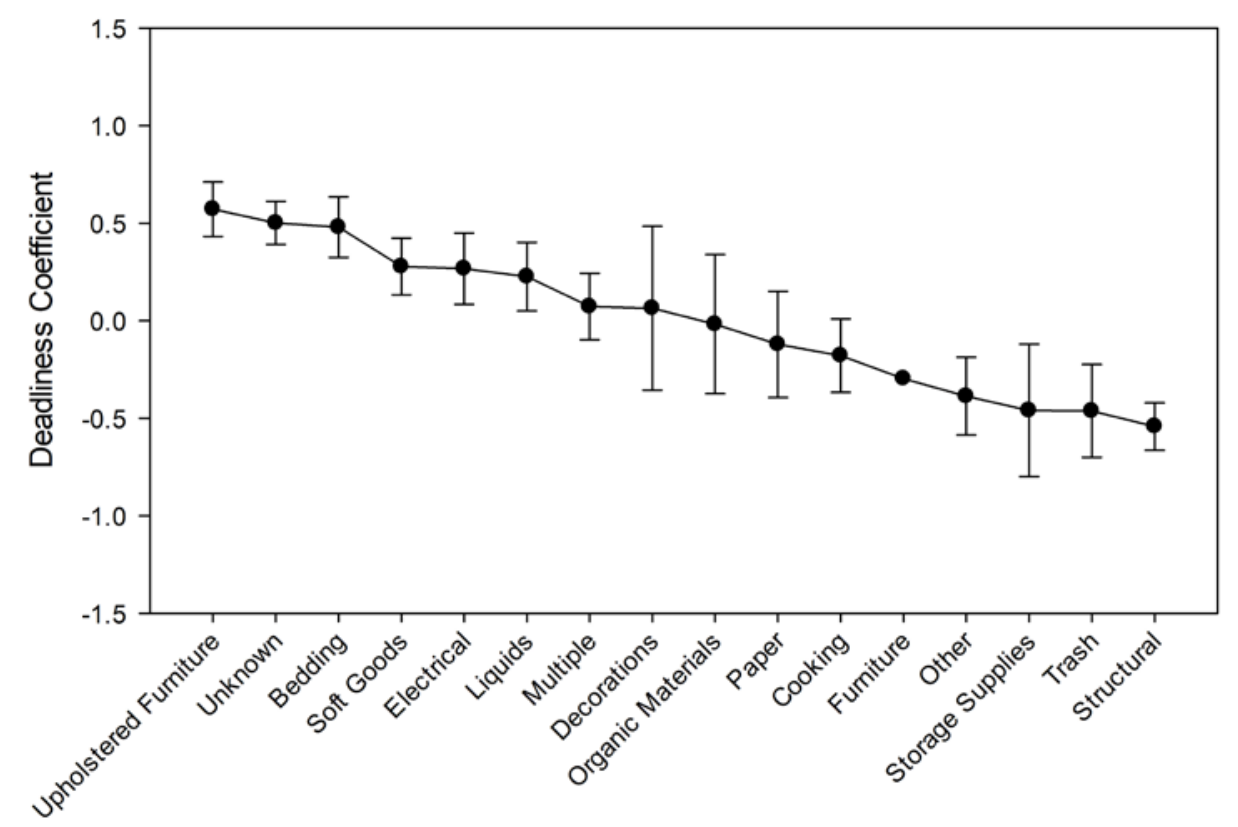

Figure 6 Relative impact of the first item ignited on the deadliness of the fire. Items listed from most deadly to least. Uncertainty bars represent $95 \%$ confidence limits.

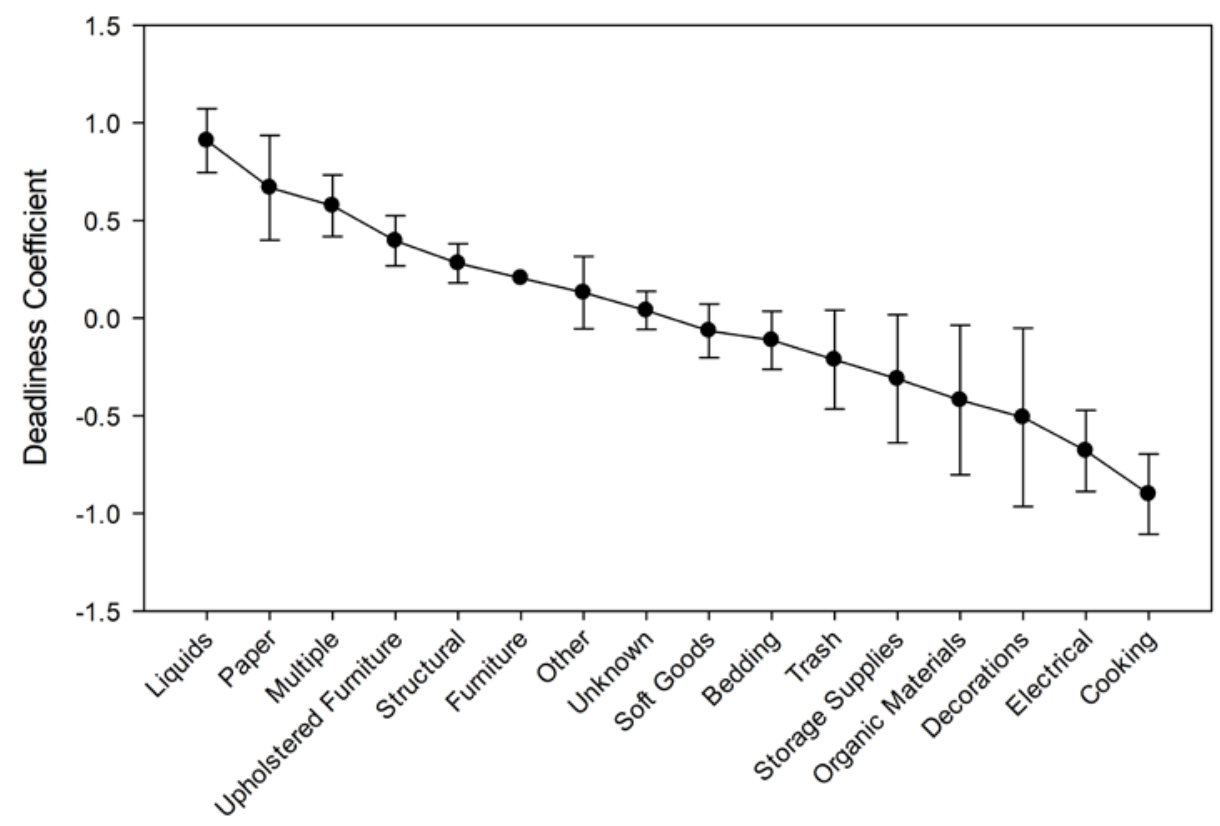

Figure 7 Relative impact of the item contributing most to flame spread on the deadliness of the fire. Items listed in order of most deadly to least. Uncertainty bars represent $95 \%$ confidence limits. 


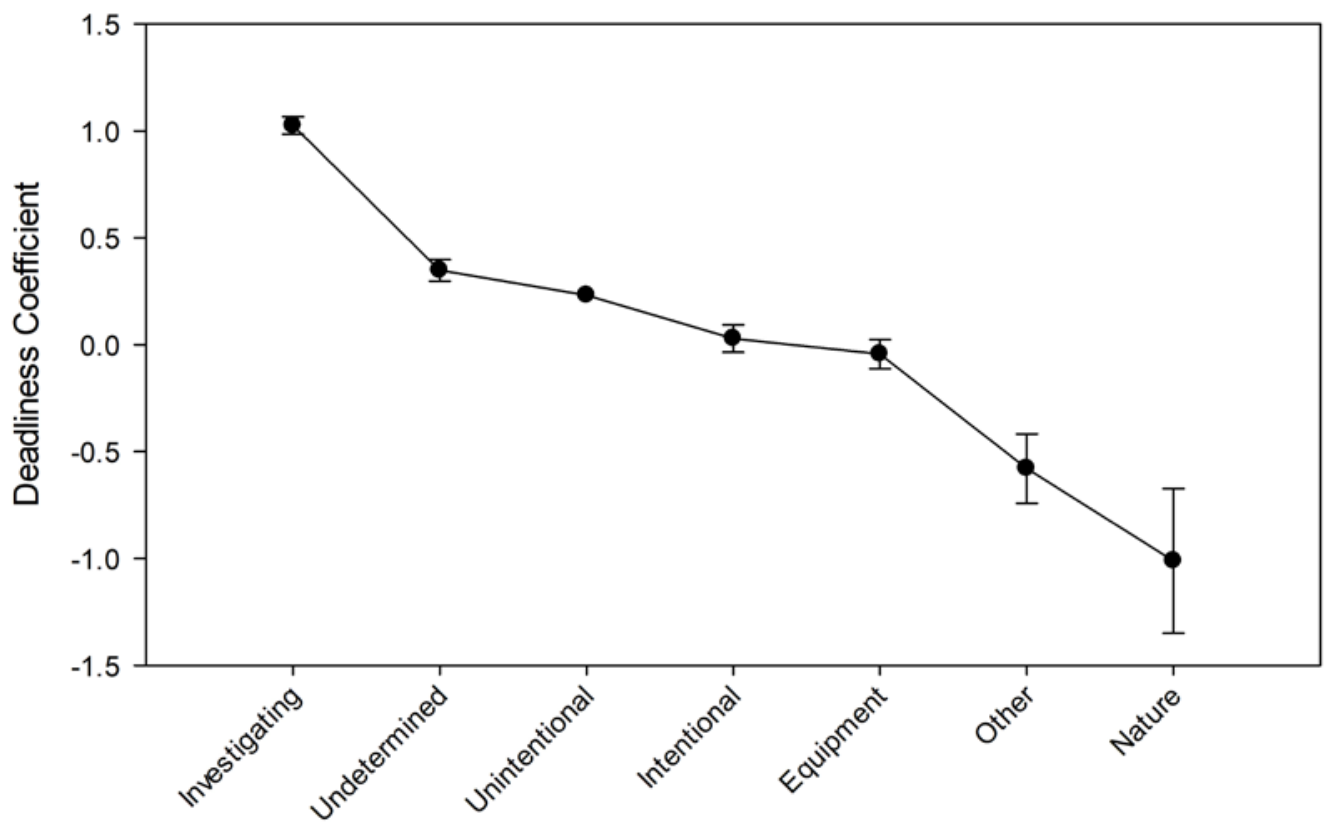

Figure 8 Relative impact of the cause of ignition on the deadliness of the fire. Causes listed in order from most deadly to least. Uncertainty bars represent $95 \%$ confidence limits.

Area of origin is the single most important factor in determining the deadliness of fires, with the most dangerous fires starting in the living room, hall, or other function areas (i.e., other than those listed). The least dangerous places for fires to start are outside or in service, equipment, or structural areas. Fires that start at night are significantly more dangerous than fires that start in the day. Fires started by smoking materials are much more dangerous. Fires that start in bedding or upholstered furniture are more dangerous than fires that start in other materials.

How does this analysis inform the scenarios we will look at in our analysis? First, note that the most dangerous room for a fire to start is the living room and the fourth most dangerous room is the bedroom. Match that up with the first and second most dangerous items first ignited are upholstered furniture and bedding and it leads to two clear scenarios. One is an item of upholstered furniture in the living room and the other is a mattress in the bedroom.

Because, smoking materials are the only the heat source that is statistically differentiated from all the other heat sources we will assume that half the fires start off smoldering and half with a small flaming ignition. The idea is to see if the method can capture the difference in the two types of ignition.

So, the analysis will consist of four scenarios that can be analyzed separately or together. Two groups will be upholstered chairs in the living room starting with either small flaming or smoldering fires, and two will be mattresses either starting with either small smoldering or flaming fires. 


\subsection{Residential Egress}

A 1935 report surveyed then-current practice in providing building exits to aid in reducing "the deplorable loss of life that occurs annually in building fires" [25]. Although primarily focused on commercial buildings since at the time, "statistical data relative to loss of life in dwellings are not readily available" [25], the report identifies a residential occupancy class that includes one- and two-family dwellings, apartment buildings, hotels and dormitories and outlines code requirements for egress for residential buildings.

As was the case in 1935, most recent research on evacuations has often focused on commercial rather than residential spaces [26, 27].

This review of residential egress is broken up into two sections. The first section looks at work based on actual emergency evacuation events and the second section looks at in-situ or laboratory evacuation experiments.

\subsubsection{Real Events}

Wood [28] conducted one of the first studies that looked at human behavior in a fire. The study looked at a collection of data from the United Kingdom that focused on fires with individuals involved. A majority of the data came from dwellings. Wood looked at the first three actions that each person took. In order of most frequent the first three were: "some firefighting action," "contact fire brigade," and "investigate fire." "Evacuated oneself" from building was the sixth most frequent action. He then broke down the actions based on the individual's familiarity with the building layout, perception of the seriousness of the fire, the degree of training, and previous experience in a fire. This allowed for a breakdown in the general behavior, how evacuations occurred, and movement through the smoke. Gender differences were also considered due to the noticeable differences in actions between male and females. One such difference was how men and women noticed a fire. Women were more likely to come to the conclusion that a fire was occurring based on seeing smoke. Men, however, more often had to see flames to be convinced of a fire. Wood also looked at circumstances people would move through smoke and how far they would travel.

Bryan [29] designed a study based on Wood's [28] that focused on fires that occurred in the metropolitan Washington, D.C/Baltimore, MD area. The majority of the fires occurred within dwellings and apartments [29]. Bryan started by looking at how the participant population became aware of the fire, and then looked at the gender distribution of this data. As Wood did, Bryan also looked at the first three actions that were completed. The actions were analyzed based on gender distribution, training, and previous fire experience After Bryan had completed his study called Project People [29], he wrote a paper [30] comparing his results with Wood's [28]. The comparison looked at the cultural variations between Americans and British in the first three actions completed during a fire. It was seen that the British populations were more likely to fight the fire as their first action, whereas Americans were more likely to notify others. Also, Americans were more likely to present behaviors related to evacuation, and the British were more likely to reenter a building.

Bryan and DiNenno [31] analyzed a fire that occurred in an apartment building in 1979. The analysis was completed through interviews and questionnaires of residents and firefighters that responded to the incident. Of the 94 responses received, 31 occupants answered that they 
were alerted to the fire by hearing the Fire Department arrive outside. One reason this was the most common answer was that the alarm system in the building had to be manually activated and was not activated until firefighters entered the building. However, the alarm only sounded for $8 \mathrm{~s}$ to $10 \mathrm{~s}$.

Occupants were also asked if they alerted anyone else before evacuating the building. Nineteen occupants responded that they did alert another occupant with the most common responses being "knocking on doors" and "waking roommates.” It was also seen that 22 occupants attempted to gain more information before evacuating with the most common response being phoning the front desk.

In 1995 Proulx [32] examined the evacuation time and movement during evacuation drills in 4 apartment buildings. The purpose was to time how long it would take individuals to start evacuating and how long it would take to evacuate the building fully. In the first 5 min in buildings 1 and 4, $76 \%$ and $70 \%$ of the occupants, respectively, had finished their evacuation, and $93 \%$ and $86 \%$, respectively, had started evacuating. In Buildings 2 and 3 the results were only $42 \%$ and $34 \%$, respectively, had started evacuation in the first 3 min and only $53 \%$ and $44 \%$ had started in the first 5 min, respectively. The reported reason was that people in buildings 2 and 3 could not hear the fire alarm, and many did not start evacuating until firefighters had arrived at the building. Another part of the study was to determine what pre-evacuation actions were taken. For Buildings 2 and 3, a common behavior was "have a look in corridor" [32]. The pre-evacuation actions show that occupants did not think immediate evacuation should take place. This may be due to a lack of training. Interviews conducted before the drills showed that evacuation drills had never taken place in three of the apartment buildings and the fourth had not held one in years. Proulx [32] also measured the amount of time it took to clear the buildings. Building 1 emptied in 3 min $5 \mathrm{~s}$ while building 4 took 4 min $38 \mathrm{~s}$. In the two buildings where hearing the alarm seemed to be problematic it took 9 min 38 s to clear building 2 and 10 min 57 s to clear building 3 . A clear contributing factor to the differing results is the pre-movement time and most important how long it took to come to the decision to evacuate. The fact that "have a look in the corridor" was an activity that so many did in buildings 2 and 3 implies strongly that information gathering was an important activity during the evacuation.

Proulx and Fahy [33] analyzed what could be classified as a good alarm or a poor alarm. This allowed them to create a graph that depicted the percentage of individuals that evacuated during a certain time. It was seen with the poor alarm that individuals were still evacuating 25 min after the alarm was activated. The review also covered the Forest Laneway Fire where occupants took a mean time of 3.18 hours to start evacuating. Twenty-four percent of occupants were unable to hear the fire alarm inside their apartments. The flames damaged the door to the exit staircase and allowed for smoke to enter and spread to other floors. By the time the occupants noticed a fire was occurring, the corridors and staircases were filled with smoke. It demonstrated that alarms should be located in a manner that allows for all occupants to hear the alarm signal when a fire occurs.

Proulx [34] analyzed a high-rise apartment fire and the behaviors that occupants demonstrated. All occupants were given a survey that asked questions such as, "How did you first become aware of the fire emergency?" [34]. Based on the data, a majority of the occupants became aware that a fire was occurring based on the voice communication system 
and fire alarm. It was found that $83 \%$ of the respondents attempted to evacuate the building. A majority did not attempt to leave immediately; $82 \%$ of the evacuating population put on warmer clothing first because of the weather conditions outside. Occupants were also asked what their perception of the smoke was and whether it made them choose not to evacuate from the building. The study concluded that occupants of high-rise apartments may be endangering themselves by not evacuating until they hear a message broadcast over the voice communications system [34].

Pauls [35] analyzed two different fires that both resulted in fatalities. The analysis shows a set of times that indicate when various actions were thought to have occurred during both fires. The first fire occurred in a 21-story apartment building on the seventh floor. It started off as a smoldering fire that took the occupant of the apartment between $15 \mathrm{~min}$ to $30 \mathrm{~min}$ to notice. The occupant attempted to extinguish the fire. However, they soon realized it would not be possible and fled the apartment. Shortly after, the apartment experienced flashover, and the building alarm started to sound. The family that experienced the two fatalities left their apartment five minutes after they heard the alarm. They attempted to evacuate down the stairs but encountered smoke on the $15^{\text {th }}$ floor and proceeded back up the stairs. Once they reached the $21^{\text {st }}$ floor, they traveled to the other staircase and descended it. The family encountered even more smoke on the $14^{\text {th }}$ floor and ascended the staircase again. They attempted to exit onto the roof, but the doors were locked. Fifty minutes after the fire department was notified of the fire they located the family. The one year old child and the mother both died due to smoke inhalation. The second fire, which was caused by ashes from an ashtray being dumped into the trash, occurred in a two-story apartment. It started on the $1^{\text {st }}$ floor of the building. This fire had over $2 \mathrm{~h}$ to build before a burning smell was detected by the occupants in the apartment where the fatality occurred. The male occupant went to go and investigate the $1^{\text {st }}$-floor apartment. While investigating, he left the apartment door open which caused smoke to start building up in the stairway. The female occupant was unable to evacuate due to the amount of smoke. She died from carbon monoxide poisoning from smoke inhalation.

Sekizawa et al. studied a fire in a 20 story high-rise apartment in Hiroshima City, Japan in 1996 [36]. The fire started on the $9^{\text {th }}$ floor and climbed the exterior of the building, going from balcony to balcony, all the way to the $20^{\text {th }}$ floor. Occupants filled out questionnaires that showed a correlation between the time delay in evacuating and previous experience with fire incidents in the building. Because previous incidents had been small and well controlled, residents were slow in their response to the fire cues. This showed the need for information that every incident will have a different outcome. The surveys also showed that $47 \%$ of occupants used the elevator to evacuate. Occupants were then asked why they chose this means of egress. Of those that used the elevator $44 \%$ did so because they used it daily. The other common answer for another $22 \%$ of occupants that used the elevator thought that it was a safer means of egress. These two answers showed that residents were unaware that elevators should not be used during a fire.

Hall [37] did an analysis of 5 years of fire incidence data. Using two different methods he analyzed how deaths and injuries would be reduced if the occupants had more time.

According to his analysis, deaths could be cut by as much as half, and injuries could be cut by two-thirds. However, these results are using some potentially optimistic assumptions. If it is assumed that extra time would only help those that were in fact evacuating, then fatalities 
and injuries would each only be reduced by one quarter. For this reason, Hall argues that we should spend less time on quantitative changes to RSET and ASET, meaning changing the amount of ASET or RSET, and focus on qualitative changes, meaning stopping fires from even starting.

Bruck and Ball [38] created a review that analyzed factors that contributed to deaths while individuals were sleeping. The risks were high levels of background noises, being a heavy sleeper, sleep deprivation, being a child, sleeping tablets, alcohol intoxication, hearing impairments, and being an older adult [38]. This led to an analysis of the fire alarms commonly used. It was seen that the typical high-frequency alarm would not wake up those in the risk group. It was suggested that low-frequency signals, as well as voice alarms, would be more likely to get a response out of individuals.

Miller and Davey [39] looked at the effect of fires on older people. Eight interviews with fire survivors were conducted for the report. The interviews looked at what the interviewee was doing before the fire broke out, the cause of the fire, and the reaction of the interviewee to the fire. Most fires occurred in the kitchen. Also, most interviewees reported now being more cautious about what they think may cause a fire in their homes [39]. The report concluded that people may not be as cautious as necessary until they have been affected by a fire.

An analysis of the Consumer Product Safety Commission third national telephone survey of unreported and non-fire department-attended residential fires was performed by Greene and Anders [40]. The report has a great deal of data about the characteristics of fires attended by fire departments and those unattended by fire departments. The headline statistics of the report is that compared to the approximately 23.7 million residential structure fires (28.3 residential structure fires per 100 households) found in the 1984 survey, residential fires have decreased significantly. The 2004-2005 survey found there were only 7.4 million residential fires, which is a rate of 6.6 residential structures per 100 households. This is a $68.7 \%$ reduction in the number of structure fires and a $76.8 \%$ decrease in the rate. The percent of fires attended by the fire department stayed about the same, going from $3.7 \%$ in the 1984 survey to $3.4 \%$ in the 2004-2005 survey.

Harpur, Boyce, and McConnell [41] found that children under the age of 5 are more at risk of a fire injuring them or being a fatality in a dwelling fire. The main reasons are young children have an underdeveloped sense of danger, are reliant on adults to escape, and spend a majority of time at home. The study [41] mentions that fire play and fire interest are risks only associated with this age group. Of the 14 incidents that were investigated, 11 were started from fire play. It was seen that $88 \%$ of the fire play incidents were influenced by a parent being a smoker, which allowed for the children to have availability to ignition sources.

Human behavior in dwelling fires has not received as much attention in the last 30 years as human behavior in public areas [42] [26]. Thompson and Wales [42] focused on accidental dwelling fires in Kent, UK. They focused on the motivations and behaviors of individuals during an accidental dwelling fire. Out of the ten interviews that were conducted, $50 \%$ of the interviewees did not realize that a fire was occurring in their home. The interviewees that did not realize a fire was occurring went to investigate the cues to determine what was happening. The Thompson and Wales [42] analysis also showed that $50 \%$ of injuries were due to interviewees attempting to fight the fire. They were surprised at the speed of the fires' 
development and how much smoke was involved. It was also seen that half of the interviewees reentered the property while the fire was occurring, supporting the observations made by Bryan [29]. By reentering, individuals are putting themselves more at risk for injury. The reasons for reentering were that they were attempting to help other individuals or rescuing pets. It was seen that more importance needs to be stressed on staying out of a building once evacuated. The authors believed that if this study was expanded, it would allow for a further understanding of the behaviors demonstrated in accidental dwelling fires and increase the importance of the subject.

\subsubsection{Experiments}

Kahn [43] also looked at the effectiveness of different cues in waking. In the study subjects were unaware of what cues would be presented, but they were told that something may change in the room. Twenty-four subjects were split into two groups. One had three different types of alarms: $78 \mathrm{dBA}, 54 \mathrm{dBA}$, and $44 \mathrm{dBA}$. The second group's cues were a $54 \mathrm{dBA}$, smoke odor, and heat. Participants were required to press a button which allowed for a timed response. It was seen that the $78 \mathrm{dBA}$ alarm had the quickest response time. Any subjects that responded to the cue were asked to identify what had woken them up. Out of the 18 subjects, only one was able to identify the alarm showing the disorientation when individuals first woke up. In the morning, the same set of questions were asked, and only one other subject could identify the smoke alarm. The experiment showed that individuals are not capable of immediately identifying a cue for fire detection.

Pearson and Joost [44] researched how various impairments could affect a person's ability to evacuate their residence. The scenarios were meant to show a set of actions that would occur during egress. The first experiment showed that wheelchair-bound adults could move large distances. However, they were slowed down by having to open doors while navigating their wheelchair. Blind subjects were at a disadvantage, being disoriented by the location of objects related to the scenarios. Tasks, where hand-eye coordination had to be applied, lead to a time-delay in egress. It was suggested that if the blind subjects had been more familiar with the environment the times to complete tasks may have been reduced.

Duncan [45] looked at the effectiveness of a smoke alarm in waking someone up. Three risk groups in New Zealand were studied. The study was used to see what the average overall response time was. Then the data was analyzed in four categories; risk groups, gender, the timeframe of alarm, and if the bedroom door was open or closed. One observation was that during timeframes in which individuals would normally be asleep, the average response time was greater. This may have been due to the extra time it took for individuals to wake up and become aware of what was occurring. The average response times were all under $30 \mathrm{~s}$ with one exception, but they all fell within "the safety period before a fire becomes life threatening” which was 2.5 min [45].

Bruck [46] researched if children would wake from hearing an alarm that is $60 \mathrm{dBA}$ at pillow level. The information that was gathered showed that $100 \%$ of the parents woke up on both nights that the alarm went off, whereas only $15 \%$ of the children woke up. The majority of the children, $55 \%$, did not wake up either night that the alarm went off. Another study [46] had parents of children age 5 years to 15 years set off an alarm for $30 \mathrm{~s}$ and report their sleeping child's reaction. The alarm did not wake $78 \%$ of the children, and no correlation 
between the bedroom door being open or closed was found. This information showed that parents cannot rely on children to wake up during a fire.

Hasofer and Bruck [47] conducted two experiments, one focusing on the response to auditory and visual cues and the other focused on olfactory cues. The first experiment had 33 participants that ranged in age from 25 years to 55 years. It focused on the auditory and visual cues, which were a shuffling noise between $43 \mathrm{dBA}$ to $45 \mathrm{dBA}$, a crackling noise between $42 \mathrm{dBA}$ to $48 \mathrm{dBA}$, and a flickering light that was 5 lux. The second experiment focused on olfactory cues and had 20 participants between the ages of 18 years to 26 years. Auditory and visual cue testing occurred in the participants' homes during their normal sleeping hours, whereas the second experiment occurred during the afternoon. By analyzing the data from both experiments, it was seen that the auditory cues had the most probability to awaken the participants. The crackling noise had the highest probability of waking both males and females. It also was seen that females were $15 \%$ more likely than males to awaken when the olfactory cue was presented. The cue that had the fastest mean time response was the shuffling noise. The study can be used to show that in a fire, an auditory cue such as an alarm is the best way to try and get an individual to wake up.

In 2004, Ball and Bruck [48] conducted a study which evaluated how a person's blood alcohol concentration (BAC) can affect response time to a fire alarm. The study used students at Victoria University, age 18 years to 25 years. Three different types of alarms were tested: a female voice, the Australian Standard Alarm, and the Temporal-Three Evacuation Signal. Individuals were tested at three different BAC levels, which were sober, 0.05 BAC, and $0.08 \mathrm{BAC}$. It was seen that the most significant difference in response time was between sober and 0.05 BAC. The results showed that even a small amount of alcohol could greatly affect a person's response time to a fire alarm.

Sleeping through an alarm is also a concern for those that are hard of hearing. Ashley [49] studied how three groups of various hearing levels would respond to three different types of alarms: a strobe light, a bed shaker, and an audible smoke alarm. The audible smoke alarm was only able to awaken those in the hearing and hard of hearing individuals. An effective alert for all three groups was the intermittent bed shaker which awakened $100 \%$ of the participants. It was also shown that the strobe light, which is seen as the alternative to the smoke alarm, was shown to be ineffective.

Kady and Davis [50] analyzed how much time it would take for 18 subjects to travel an escape route. There were nine male and nine female subjects that were split into three groups normal, overweight, and obese. The subjects had to crawl two different escape routes, a straight route and an indirect route; each was $100 \mathrm{ft}$. The purpose was to determine if there was a time difference. It was found that with any body composition, it took more time for the indirect route to be navigated. The average time differences for the males were $2.62 \mathrm{~s}, 4.09 \mathrm{~s}$, and $6.36 \mathrm{~s}$ for normal, overweight, and obese groups respectively. The average time differences for the females were $2.61 \mathrm{~s}, 2.85 \mathrm{~s}$, and $0.56 \mathrm{~s}$.

Smoke can affect the walking speed and thinking ability of an individual. Jin and Yamada [51] analyzed how far someone could walk in a smoke-filled corridor. As the individual proceeded down the hallway they were given arithmetic problems, and the amount of correct answers was collected. A control group was set by having subjects walk down the hallway 
without any smoke. It was determined that as individuals went farther down the smoke-filled corridor, the walking speed and amount of correct answers decreased. This shows that fire smoke can negatively affect a person's thinking ability and walking speed, which can affect how long it takes someone to evacuate a building.

\subsubsection{Discussion}

There are significant features that make residential egress qualitatively different from commercial egress. One example is that commercial egress can often be described in terms of crowd dynamics while single family homes must more frequently be described in terms of individual behavior.

One such behavior is occupants fighting the fire. As noted above, Greene and Anders [40] found that only $3.4 \%$ of residential fires were attended by the fire department. The implication is residents successfully extinguish the majority of home fires. The value of the kind of analysis presented in this work is understanding the impact of the new technology on occupant behavior including but not limited to fighting the fire.

In conclusion, a number of articles on residential egress were reviewed. It became clear through the review that there was no consensus in terms of a model for residential egress. Therefore, it would not be practical to model residential egress in any realistic way for this report, so it has been left out of the final analysis.

\section{Method}

Bukowski [4], Clarke et al. [6], and more recently, building codes [52] and engineering handbooks [53] provide a structure for a fire hazard analysis that can be used to characterize the relative performance of two sets of fire scenarios. They outline several key areas that need to be addressed in the analysis. These include definition of:

1. Community / Building / Occupant characteristics

2. Fire scenarios

3. Analysis variables / Criteria for comparisons

4. Statistical analysis of calculation results

Much of the purpose of this report is to better understand and quantify the general process for defining each of these areas to better represent an overall hazard analysis with statistically defensible results and analysis.

\subsection{Community / Building / Occupant Characteristics}

Step one is to develop a method to characterize the community being considered. The question is what constitutes a full characterization of a community and how is that accomplished. It is likely that there are holes in the available data, so it is important to understand and document any assumptions that would have to be made and the extra analysis that must be done. An example is sensitivity testing of the assumptions, to ensure confidence in the resulting analysis. 


\subsubsection{Distribution of Houses}

To characterize an average U.S. community, the starting point taken is the American Housing Survey (AHS) performed every other year by the United States Census Bureau for the Department of Housing and Urban Development (HUD) [54]. The AHS has been around since 1973 in one form or another and is taken every odd number year with the purpose "to provide a current and continuous series of data on selected housing and demographic characteristics.” [54]

Information from the AHS can be presented in a variety of tables. For this project, the base data starts with the total floor area in square feet ${ }^{2}$ of the homes. The data is sorted into a number of bins that divide up total area from $0 \mathrm{ft}^{2}$ to more than $4000 \mathrm{ft}^{2}\left(272 \mathrm{~m}^{2}\right)$ and yields a number of houses in each bin. For example, of the estimated 92676000 houses reporting total area in the survey, 23563000 or $27.6 \%$ had a floor area between $1000 \mathrm{ft}^{2}\left(93 \mathrm{~m}^{2}\right)$ and $1499 \mathrm{ft}^{2}\left(139 \mathrm{~m}^{2}\right)$. From this a smooth cumulative density function (CDF) can be constructed as seen in Figure 9. As can be deduced from Figure 9, the maximum total floor area considered is $5000 \mathrm{ft}^{2}\left(465 \mathrm{~m}^{2}\right)$. The actual survey's final bin is a total area of $4000 \mathrm{ft}^{2}(272$ $\mathrm{m}^{2}$ ) or more. However, since less than $3.2 \%$ of houses were over $4000 \mathrm{ft}^{2}\left(272 \mathrm{~m}^{2}\right)$ and there was no real information on the distribution of larger houses, the decision was made to cut the total floor area off at $5000 \mathrm{ft}^{2}\left(465 \mathrm{~m}^{2}\right)$.

The data are further broken down to give the number of bedrooms for each house in a particular bin. Continuing with the example of the homes between $1000 \mathrm{ft}^{2}\left(93 \mathrm{~m}^{2}\right)$ to $1499 \mathrm{ft}^{2}\left(139 \mathrm{~m}^{2}\right)$, there are only 20000 or $0.08 \%$ of the homes that do not have a bedroom, while 12510000 or $53.1 \%$ of the homes have three bedrooms. Finally, for a given number of bedrooms in any given total area bin, the AHS gives the distribution of the number of rooms the house has. For the 12510000 homes with total area between $1000 \mathrm{ft}^{2}\left(93 \mathrm{~m}^{2}\right)$ and $1499 \mathrm{ft}^{2}\left(139 \mathrm{~m}^{2}\right)$ and three bedrooms, 5918000 or $47.3 \%$ have five total rooms, not counting bathrooms. From these data, a series of conditional CDFs can be created to allow definition of a set of houses with a random total area, a random number of bedrooms, and a random total number of rooms, which are all consistent with the housing data.

\footnotetext{
${ }^{2}$ The AHS provides data in square feet. While NIST's preferred units would be square meters we use square feet to make it easier to go back to the original reference.
} 


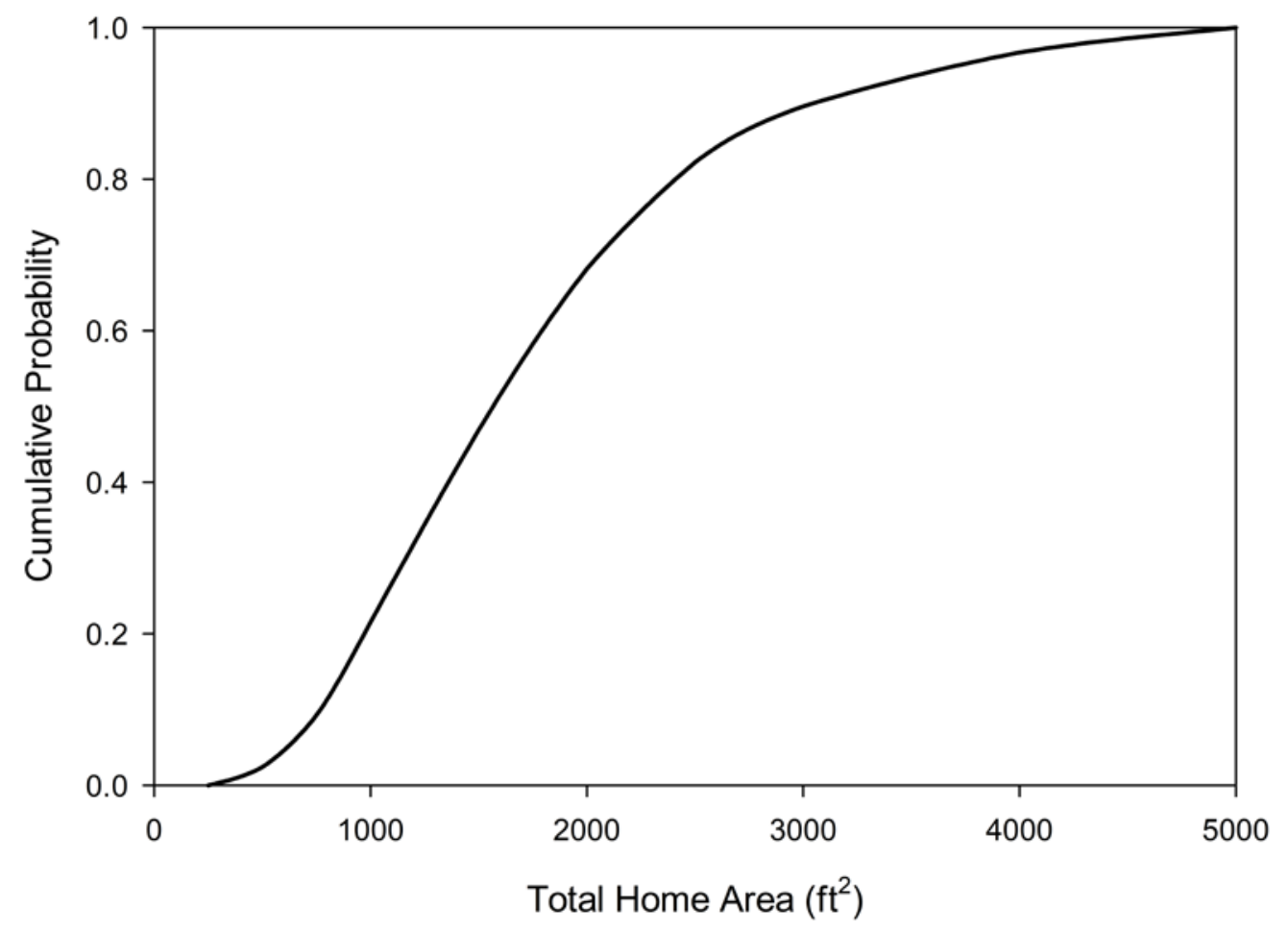

Figure 9 Cumulative Distribution Function (CDF) for the total area of the home.

\subsubsection{Determining the Floorplan}

Once the total square footage and number of rooms was determined, a floorplan was generated according to a few simple rules. The total area was divided amongst the rooms randomly with an assumed minimum room area of $20 \mathrm{ft}^{2}\left(1.9 \mathrm{~m}^{2}\right)$. A room aspect ratio of width divided by length was assumed to be 0.75 for all rooms. This assumption should not significantly affect the results of zone model simulations such as are performed in CFAST because the only impact is on the amount of energy transferred to the walls and this is a relatively small amount of energy as compared to other sources and sinks of energy, for example the fire and openings such as doors and windows.

To connect the rooms, a simple method was used. Each pair of rooms was considered and assigned a $50 \%$ chance of being connected. While this means that some scenarios use houses that have many connections, it still leaves the possibility of rooms and even sections of the house being closed off from the fire, which is assumed to be the impact of closed doors. This random approach to generating connections has a probability of resulting in a floorplan that cannot be instantiated in a single story. More technically, if the floorplan is thought of as a graph with the rooms as vertices and the connections as edges, some of the randomly generated graphs will be nonplanar for cases with more than four rooms. A planar graph is one that can be drawn on a piece of paper and none of the edges cross. The probability of generating a nonplanar floorplan increases as the number of rooms increases. In order to 
eliminate such nonphysical cases from the analysis, any randomly generated floorplan can be checked for planarity and rejected if necessary and replaced by a new randomly generated floorplan.

\subsection{Modeling Smoke Detectors}

The current version of the fire model CFAST [11] uses a default alarm threshold based on a temperature rise of $5{ }^{\circ} \mathrm{C}$, from a study of Bukowski and Averill [55], or a smoke concentration of $8.0 \% / \mathrm{ft}(23.0 \% / \mathrm{m})$ obscuration, based on an evaluation of smoke detector performance by Milke, Mowrer, and Gandhi [56]. These estimates represent average alarm threshold values without any predetermination of the type of alarm or smoke properties. A more detailed model is required in this case study to account for the variation in smoke alarm response, given differing smoke properties for flaming and smoldering upholstered furniture combustion and given differing alarm sensor types.

A statistical smoke alarm activation model was developed for upholstered furniture containing polyurethane foam [57]. The statistical model is represented as a log-normal distribution with a geometric mean and geometric standard deviation. The specific distribution depends on the smoke alarm type, and the mode of combustion prior to alarm activation. Thus, the distributions are more refined than the current default alarm threshold value in CFAST. Comparison of the statistical model results to real-scale furniture mockup experiments shows good agreement with flaming scenarios and the correct trend in smoldering scenarios. The data used to develop the statistical model comes from experiments recently conducted at NIST on the response of a number of smoke alarms to the new smoldering and flaming polyurethane foam tests specified in ANSI/UL 217-2015 [2]. A total of 45 different alarm designs, representing alarms currently available in the U.S. (23 containing ionization sensors and 22 containing photoelectric sensors), were examined in the study. An additional benefit to using the results from the ANSI/UL 217-2015 test configuration is that the performance of new alarms meeting the standard can be directly translated into statistical models for those alarms. This would allow direct comparison between newer smoke alarms and pre-2015 edition smoke alarms. Currently, no alarms that meet the standard are available. Therefore, an estimate was made for the geometric mean and geometric standard deviation of the obscuration at alarm for flaming and smoldering upholstered furniture fires for new smoke alarms that would meet the ANSI/UL 217-2015 standard. Table 1 give the means and standard deviations for ionization, photoelectric and new smoke alarms for flaming and smoldering upholstered furniture fires. 
Table 1 Parameters for smoke alarm statistical models

\begin{tabular}{|c|c|c|c|c|}
\hline \multirow{2}{*}{ Alarm Type } & \multicolumn{2}{|c|}{ Flaming Fire Obscuration } & \multicolumn{2}{c|}{ Smoldering Fire Obscuration } \\
\cline { 2 - 5 } & $\begin{array}{c}\text { Geometric } \\
\text { Mean (\%/ft) }\end{array}$ & $\begin{array}{c}\text { Geometric } \\
\text { Std. Dev. }\end{array}$ & $\begin{array}{c}\text { Geometric } \\
\text { Mean (\%/ft) }\end{array}$ & $\begin{array}{c}\text { Geometric } \\
\text { Std. Dev. }\end{array}$ \\
\hline Ionization & 2.1 & 1.3 & 10.0 & 1.2 \\
\hline Photoelectric & 6.7 & 1.3 & 3.5 & 1.7 \\
\hline \multicolumn{2}{|c|}{} & 1.3 & 5.0 & 1.3 \\
\hline
\end{tabular}

\subsection{Fire Scenarios}

As was discussed in section 2.2 there are two fire scenarios of particular importance in residential fire loss statistics, an upholstered chair fire in a living room and a mattress fire in a bedroom. For all fire scenarios, the fire was assumed to start as a small smoldering or flaming incipient fire in a single furniture item (either an upholstered chair or a mattress), which at some point transitioned to vigorous burning. With this, we can look at both the performance of fire detection devices and the tenability conditions in rooms remote from the fire.

In determining distributions to sample from, a basic assumption was made: all the underlying data would result in simple uniform or triangular distributions. There are two reasons for this. The first is the reality that these quantities are generally confined to a simple range. For example, the peak heat release rate (HRR) is limited by the size and shape of the object burning. Unless the conditions allow an object to self-extinguish there is going to be a minimum peak HRR above $0 \mathrm{~kW}$. Because of the limits of surface area, total fuel, and radiation to the object, there is a physical maximum peak HRR that can be achieved. The second reason for the simple distributions was the limited data available from which to draw inferences on more complex distributions.

To determine the fire defined for each test case, four parameters were sampled from distributions. Because we were looking at the impact of different alarm types, we decided to focus on fires that have an initial slow growth phase due to a very small ignition. This means started with a linear growth phase that grew from $0 \mathrm{~kW}$ at a specified linear rate and for a specified time. At the end of the linear growth phase the fire transitioned to a more vigorous burning phase characterized by a t-squared profile and a peak HRR. The four parameters were the linear growth rate, the time of the linear growth phase, the peak HRR, and the time when the peak HRR is achieved.

This basic model for representing the fire was taken from Cleary [58] who conducted a series of real-scale experiments with smoldering and flaming ignition of upholstered furniture items. Among the data collected were ignition delay (for flaming fires), time to flaming 
transition (for smoldering fires), estimates of HRR at the time of transition from the initial, assumed linear growth phase to a t-squared growth phase (these three values define the initial linear growth phase for both fire types as a linear growth time, $t_{L}$, and a HRR value at the end of the linear growth phase, $\dot{Q}_{L}$ ), and time to a HRR of $1055 \mathrm{~kW}$ (a value consistent with the typical definition of t-squared fires). With exceptions, fires grew at a roughly medium tsquared fire growth rate (typically defined as a $300 \mathrm{~s}$ time to $1055 \mathrm{~kW}$ HRR). Peak HRR was not reported. Table 2 summarizes the test data collected, with the materials that made up the furniture items listed in the first column.

Table 2. Ignition delay and growth rate of upholstered furniture fires from reference [58].

\begin{tabular}{|c|c|c|c|c|c|c|}
\hline \multirow[t]{2}{*}{ Material } & \multirow[t]{2}{*}{ Experiment } & \multirow{2}{*}{$\begin{array}{l}\text { Ignition } \\
\text { Mode }\end{array}$} & \multirow[t]{2}{*}{ Location } & \multicolumn{2}{|c|}{ Linear Phase } & \multirow{2}{*}{$\begin{array}{c}\text { Time to } \\
1055 \mathrm{~kW}\end{array}$} \\
\hline & & & & $t_{L}(\mathrm{~s})$ & $\dot{Q_{L}}(\mathrm{~kW})$ & \\
\hline PET/LD & 4 & Flaming & LR & 150 & 10 & 271 \\
\hline PET/LD & 17 & Flaming & LR & 200 & 25 & 212 \\
\hline PET/LD & 19 & Flaming & $\mathrm{LR}$ & 175 & 25 & 201 \\
\hline $\mathrm{PET} / \mathrm{HD}$ & 8 & Flaming & $\mathrm{BR}$ & 260 & 30 & 223 \\
\hline $\mathrm{PET} / \mathrm{HD}$ & 10 & Flaming & $\mathrm{BR}$ & 280 & 30 & 161 \\
\hline $\mathrm{PET} / \mathrm{HD}$ & 24 & Flaming & $\mathrm{BR}$ & 240 & 25 & 207 \\
\hline PET/LD & 7 & Flaming & $\mathrm{BR}$ & 180 & 25 & 205 \\
\hline PET/LD & 11 & Flaming & $\mathrm{BR}$ & 160 & 10 & 182 \\
\hline CT/LD & 1 & Flaming & LR & 1200 & 25 & 312 \\
\hline CT/LD & 6 & Flaming & $\mathrm{LR}$ & 220 & 25 & 285 \\
\hline CT/LD & 18 & Flaming & $\mathrm{LR}$ & 800 & 25 & 190 \\
\hline CT/LD & 5 & Smoldering & LR & 6180 & 10 & 191 \\
\hline CT/LD & 22 & Smoldering & LR & 5790 & 10 & 177 \\
\hline $\mathrm{CT} / \mathrm{HD}$ & 16 & Smoldering & $\mathrm{LR}$ & 5240 & 10 & 177 \\
\hline $\mathrm{CT} / \mathrm{HD}$ & 21 & Smoldering & $\mathrm{LR}$ & 6210 & 10 & 160 \\
\hline $\mathrm{CT} / \mathrm{HD}$ & 23 & Smoldering & LR & 5180 & 10 & 163 \\
\hline $\mathrm{CT} / \mathrm{LD}$ & 12 & Smoldering & $\mathrm{BR}$ & 4840 & 10 & 174 \\
\hline CT/LD & 15 & Smoldering & $\mathrm{BR}$ & 10910 & 20 & 131 \\
\hline CT/LD & 2 & Smoldering & $\mathrm{BR}$ & 6125 & 10 & 298 \\
\hline CT/LD & 9 & Smoldering & $\mathrm{BR}$ & 6790 & 10 & 228 \\
\hline
\end{tabular}

PET = Polyester fabric, CT = Cotton fabric, LD = Low density foam, HD = High density foam, LR = Living Room, BR = Bedroom

In this study, both flaming and smoldering fires are modeled with an initial linear growth incipient fire phase (before vigorous burning) followed by a t-squared growth curve. For flaming fires, the average of the linear phase duration was $207 \mathrm{~s} \pm 46 \mathrm{~s}^{3}$, and the average and range of the transition HRR was $23 \mathrm{~kW} \pm 7 \mathrm{~kW}$. For smoldering fires, the average and range of the linear phase duration was $6363 \mathrm{~s} \pm 1812 \mathrm{~s}$, and of transition HRR was $11 \mathrm{~kW} \pm 3 \mathrm{~kW}$. After the linear growth phase, the fires grew at a medium t-squared rate, taking $222 \mathrm{~s} \pm 47 \mathrm{~s}$

\footnotetext{
${ }^{3}$ For consistency with typical uncertainty results reported for fire models, all uncertainty values in this report are expressed as one standard deviation.
} 
to reach $1055 \mathrm{~kW}$ for flaming fires and taking $189 \mathrm{~s} \pm 48 \mathrm{~s}$ to reach $1055 \mathrm{~kW}$ for smoldering fires.

For upholstered chairs, Babrauskas [59] compared predictive models of upholstered furniture fire growth to a range of available experimental data. Peak HRR for the upholstered furniture ranged roughly from $200 \mathrm{~kW}$ to $2500 \mathrm{~kW}$.

The type of distribution was determined by inspection of the data available. For example, consider the length of time for the linear growing phase for the flaming chair. Figure 10 shows the time of the linear phase for each of the flaming chair tests in Table 2. Most of the times are in a narrow range from $150 \mathrm{~s}$ to $280 \mathrm{~s}$, with only two times outside of the cluster. Clearly it would not be appropriate to represent this data set with a uniform distribution, and so we use a triangular distribution as seen in Figure 11.

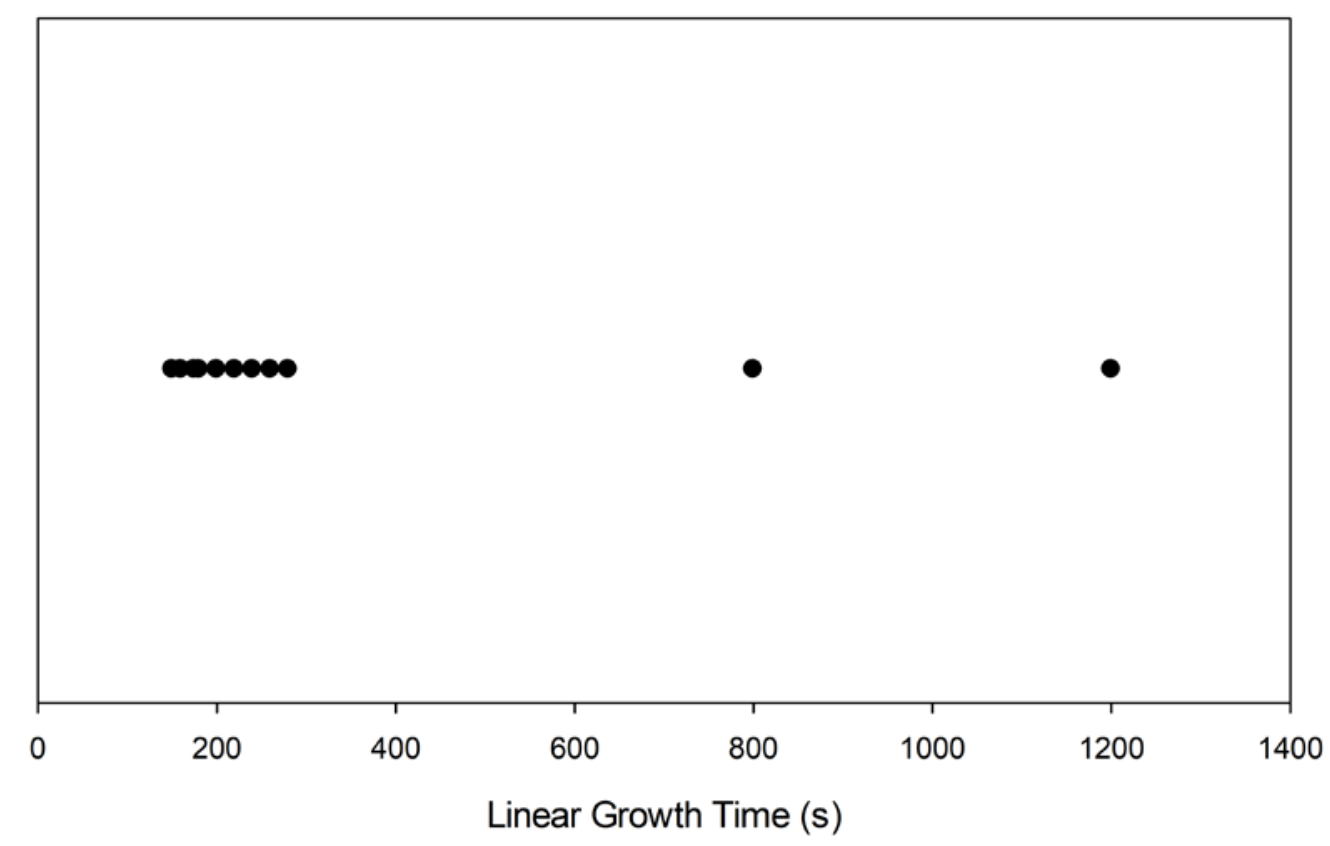

Figure 10 Distribution of linear growth time for flaming chair 


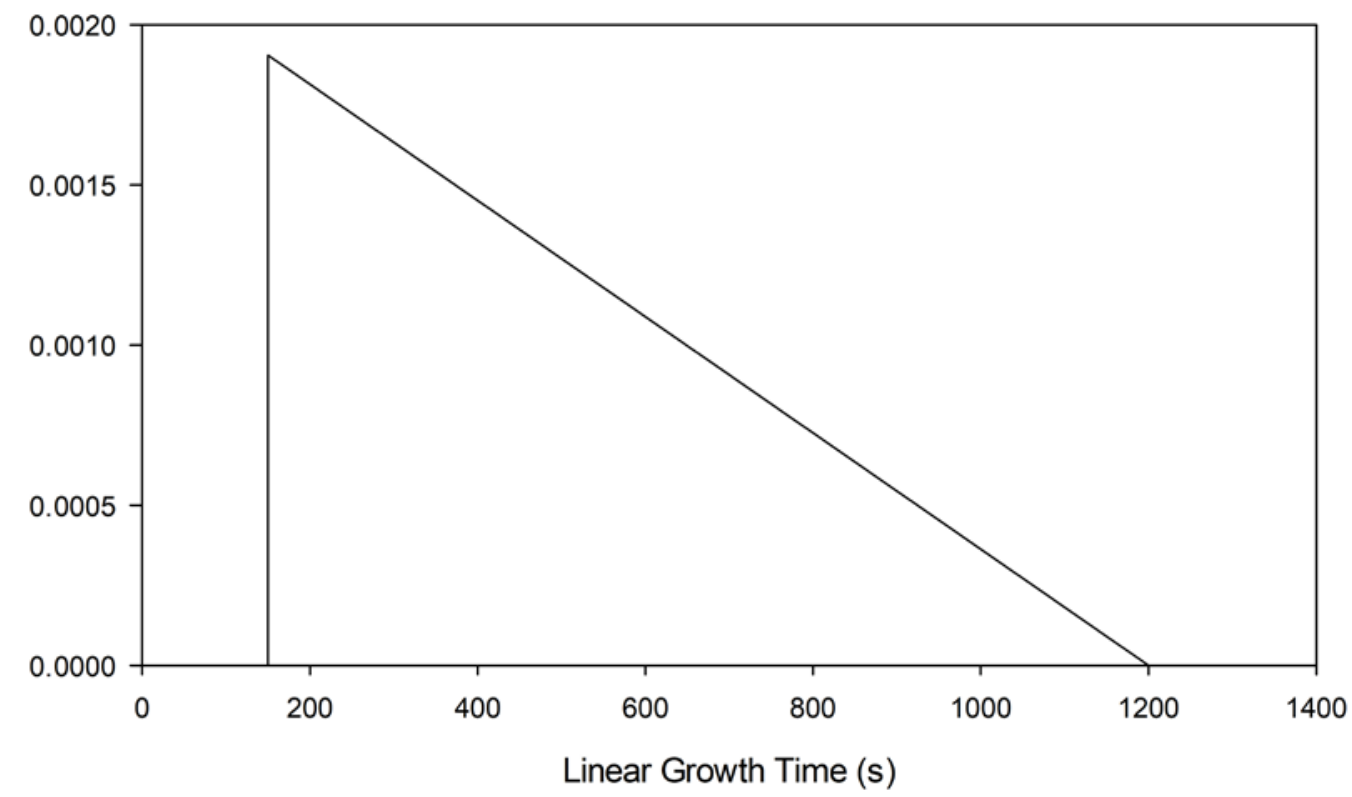

Figure 11 Triangular distribution for linear growth time for flaming chair

For mattresses, Olhemiller and Gann [15] provide experimental results for flaming ignition fires of three mattress types (discussed earlier in section 2.1). The M1 type represented currently available mattresses (termed "Old Matresses" in this report), while M3 and M5 were two different types of mattresses designed to meet reduced HRR standards (combined, termed "New Mattresses" in this report). The mattress peak HRR values and times to peak HRR are reported in Table 3. Note that half the experiments were conducted under an open hood and half were conducted in an enclosure designed to simulate a bedroom.

In many of the tests, there were two peaks. In the table both peaks are included, if applicable, with the smaller peak in parentheses. For our analysis, only the large peak values were used.

The fuel properties were not varied according to a distribution. We used typical values for upholstered furniture, consistent with Cleary [58]: heat of combustion $13000 \mathrm{~kJ} / \mathrm{kg}$, soot yield $0.01 \mathrm{~kg} / \mathrm{kg}$ fuel for flaming fire, soot yield $0.23 \mathrm{~kg} / \mathrm{kg}$ fuel for smoldering fires, and CO yield $0.05 \mathrm{~kg} / \mathrm{kg}$ fuel. The chemical formula for the fuel was defined as carbon $=9$, chlorine $=0$, hydrogen $=6$, nitrogen $=0.2$, and oxygen $=2$. 
Table 3 Peak HRR and time to peak HRR for flaming ignition of three mattress types both under a hood and in a room

\begin{tabular}{|l|l|l|l|l|}
\hline Design & Size & Location & Peak HRR $\left(\mathrm{kW} / \mathrm{m}^{2}\right)$ & Time to Peak (s) \\
\hline M3 & Twin & Hood & $166,(155)$ & ca. 390, (ca. 710) \\
\hline M3 & Twin & Hood & $(165), 187$ & (ca. 360), ca. 660 \\
\hline M3 & King & Hood & 240 & 640 \\
\hline M3 & King & Hood & 290 & 380 \\
\hline M5 & Twin & Hood & $(230), 655$ & $(405), 715$ \\
\hline M5 & Twin & Hood & $(255), 595$ & $(420), 880$ \\
\hline M5 & King & Hood & $775,(660)$ & $700,(1240)$ \\
\hline M5 & King & Hood & 620 & 1160 \\
\hline M1 & Twin & Hood & 2275 & 400 \\
\hline M1 & Twin & Hood & 2310 & 435 \\
\hline M1 & King & Hood & 3370 & 370 \\
\hline M1 & King & Hood & 3850 & 305 \\
\hline M3 & Twin & Room & $(140), 150$ & $(490), 680$ \\
\hline M3 & Twin & Room & $(130), 190$ & $(430), 710$ \\
\hline M3 & King & Room & 390 & 550 \\
\hline M3 & King & Room & 420 & 525 \\
\hline M5 & Twin & Room & $(300), 1670$ & $(600), 915$ \\
\hline M5 & Twin & Room & $(225), 190$ & $(420), 1140$ \\
\hline M5 & King & Room & $(880), 955$ & $(445), 970$ \\
\hline M5 & King & Room & $(700), 815$ & $465,(1165)$ \\
\hline M1 & Twin & Room & 3850 & 365 \\
\hline M1 & Twin & Room & 2995 & 350 \\
\hline M1 & King & Room & 3465 & 305 \\
\hline M1 & King & Room & 4620 & 335 \\
\hline & & & & \\
\hline
\end{tabular}

\subsection{Monte Carlo Modeling with CFAST}

The preceding discussion provides a statistical description of residential fire scenarios. The hazard of a specific individual scenario may be predicted by the use of an appropriate fire model. However, a rigorous characterization of the fire hazard faced by a community will necessarily be statistical. It is therefore necessary to propagate the probability distributions describing the scenarios through the fire model to obtain a statistical characterization of the fire hazard. A straightforward approach to achieving this propagation is through use of Monte Carlo simulation. Monte Carlo simulation is simply the repeated execution of a model using random samples of stochastic model inputs. In addition to its simplicity, Monte Carlo simulation has the advantage of converging at a rate of $1 / \sqrt{N}$ (with $N$ being the number of model simulations) regardless of the dimensionality of the problem (i.e., the number of stochastic model inputs). 
The approach described in the following is a specific example of a general approach. The Monte Carlo approach to characterizing hazard can, in principle, be applied to any set of statistically described fire scenarios. Furthermore, any fire model can be used to execute the Monte Carlo simulations. For the purposes of this report, CFAST was chosen as an appropriate model for use with a Monte Carlo simulation of the fire scenarios described above.

The process can be decomposed into four distinct parts:

1. Scenario sampling,

2. CFAST input file generation,

3. CFAST execution, and

4. Post-processing of CFAST outputs.

The process was implemented as a Python script wrapped around a CFAST executable. First, the scenario was randomly sampled from the probability distributions described above. A sampled scenario consists of a complete description of the home layout, fire, and smoke alarm response characteristics. This sampled scenario is stored as a Python data structure and input into a function that generates a CFAST input file. The governing script then calls the external CFAST executable, opens several of the resulting output files and extracts the information relevant to the hazard associated with the sampled scenario. In a Monte Carlo simulation, this procedure is repeated many times, and the results are interpreted as a statistical representation of the fire hazard.

The CFAST simulations can take a varying amount of time depending on the sampled scenario. In some cases, the sampled fire is simply of longer duration and takes longer to simulate. In other cases, the system of differential equations underlying CFAST will require smaller time steps, and therefore a larger number of time steps, to maintain stability. Cases in which the number of time steps exceeded a critical value were stopped prior to completion to avoid impractically long simulation times. These cases accounted for around $5 \%$ to $10 \%$ of the total number of simulations. The impact of these failed cases on the results is discussed in Section 4.1.

In any case, the procedure described above is easily parallelizable since each Monte Carlo run is independent of the others. This allowed for a large amount of data to be produced in a relatively short amount of time.

\subsection{Analysis Variables}

Choosing modeling results for analysis depends on the goals of the hazard analysis and the particular technology under study. In the most general sense, this means understanding what results are indicative of improved fire safety. This is not a cost benefit analysis, which is a superset of this analysis and outside the scope of this project. To improve fire safety generally means reducing the deaths and injuries due to a fire. So, the goal is to identify which variables, that can be calculated, would give an indication that a new technology, in our case the new smoke alarm technology, will reduce deaths and injuries. 
To determine if occupants would escape a fire unharmed, we would need to be able to properly distribute a population in the houses, including understanding the demographics of the population, their position in the house, and their activity. For example, from the analysis of fire data in section 2.2.2, the deadliest times for fire is the late night and early morning (presumably when most people are asleep). Thus, we would need to be able to calculate what people do in reaction to cues of a fire, e.g., smelling smoke or hearing an alarm. The actions are quite varied and include fighting the fire [28] and notifying others [29]. Finally, we would need to determine the impact of the fire's heat and toxic gases on the occupants as they react to the fire.

As discussed in section 2.3.3, currently available egress models do not take into account all the varied activity of occupants in residential fires. However, there are simple surrogate measures that can act as zeroth order models of occupant behavior to fires. The first is to determine a reasonable maximum required egress time that is independent of the fire scenario. In NIST's fire safety analysis of smoke alarms in homes [60] a value of $90 \mathrm{~s}$ was seen to be a reasonable estimate of the egress time of a young family at night in reaction to an alarm sounding. While that time was for a manufactured house and a specific two-story structure, we will use the same value as a base time. To better understand the impact of a residential egress model and the fact that some groups of occupants will evacuate faster or slower than others, we will consider some multiples of the base $90 \mathrm{~s}$. In this analysis we will also consider the ability of occupants to fully evacuate the house in $45 \mathrm{~s}, 90 \mathrm{~s}, 180 \mathrm{~s}$, and 270 s. For an actual analysis, a better understanding of egress time in residences would be required.

The second behavior that would be good to model is if an occupant successfully fights the fire, as this eliminates the need to evacuate the house. As was discussed in section 3.3, all the fire scenarios used in this analysis have a linear growth incipient fire phase. We will assume that it is much easier to put the fire out if it is discovered before the vigorous burning (tsquared profile) starts. As a simple model, following the example of egress, we will assume that if the alarm sounds at least $30 \mathrm{~s}, 60 \mathrm{~s}, 120 \mathrm{~s}$, and $180 \mathrm{~s}$ before the end of the linear growth phase, there is a high probability that the fire would be put out. Otherwise there is a low probability that the fire is extinguished.

To quantify the impact of the fire's heat and toxic gases on people, ISO International Standard 13571 details calculations of tenability resulting from asphyxiant gases and convected heat from fires [17]. These calculations are implemented in the CFAST model to account for the impact of $\mathrm{CO}, \mathrm{CO}_{2}$, and $\mathrm{HCN}$ with the limitation that the tenability calculations are made at fixed locations within a room. Still, these calculations can be used as a benchmark. For example, a successful evacuation can be assumed if tenability conditions in rooms remote from the fire are less than the critical values up to $90 \mathrm{~s}$ after an alarm sounds. We will track three critical values of fractional effective dose (FED) ${ }^{4}: 0.3,0.5$, and 1.0 , all

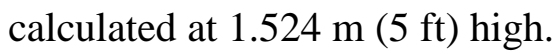

\footnotetext{
${ }^{4}$ The fractional effective dose (FED) is a measure of the impact of toxic gases and heat on individuals exposed to a fire. It is defined as the dose of a toxic product acquired during a short period of time, expressed as a fraction of the dose required to cause incapacitation or death during that time interval. Typical values are 0.3 as an incapacitating dose for sensitive populations, 0.5 as an incapacitation dose for normal populations, and 1.0 as a lethal dose for normal populations.
} 
We will also consider another indicator of hazard, smoke optical density, to be consistent with previous NIST studies [60]. The hazard condition is when any room in the floor plan

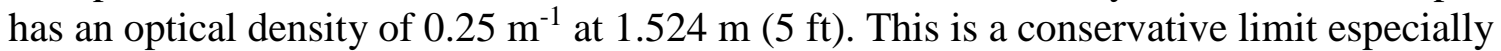
because it is for any room, not necessarily along any occupant's egress path. Still, we include the indicator not only to be consistent with previous studies, but also because egress should not be considered safe just because it happens that a single occupant does not encounter through smoke. Rather, it should be the case that there is no chance of crossing through smoke, and that no room in the house, other than the fire room, has enough smoke to inhibit egress.

Clearly, these models of occupant behavior and movement are simplistic and have somewhat arbitrary criteria in trying to account for the actions of occupants during a fire. It is not clear how much confidence we can have in the analysis or its ability to represent real performance. Also, it does not necessarily give a complete or accurate understanding of how the new technology is working. Thus, this report is mainly used as a surrogate pending a better quantitative understanding of human behavior in residential fires.

For our case study, the question is how the new smoke alarms respond compared to current ionization and photoelectric alarms. To better understand this, we will look at the difference in activation times between the alarms as well as the difference in available egress time between the alarms.

\section{Results}

A part of understanding the Monte Carlo process is to analyze the results. In the following sections, examples of data analysis are discussed with the aim of better understanding the necessary steps of the process.

Key initial questions for the analysis include how many cases could realistically be run and how many cases are actually needed for a valid analysis. Over the course of approximately 17 days we were able to generate, run and process 57500 cases. In the following sections we will discuss the results of those simulations including a discussion of how many runs were needed for a valid statistical analysis of the data in section 4.2.

\subsection{Observations}

To obtain 57500 valid cases, we generated 62902 cases. 5402 cases were discarded because they failed with mathematical error in the model or because the cases took too long to run in a reasonable time.

To better understand the distribution of cases, a number of histograms of input data are presented in Figure 12 to Figure 19. Figure 12 shows the distribution of fires that were run. The fire distributions were designed so that each fire type would be equally possible. However, from Figure 12, we see that the old mattress type fires are underrepresented and that the new mattress type fires are a little overrepresented. 


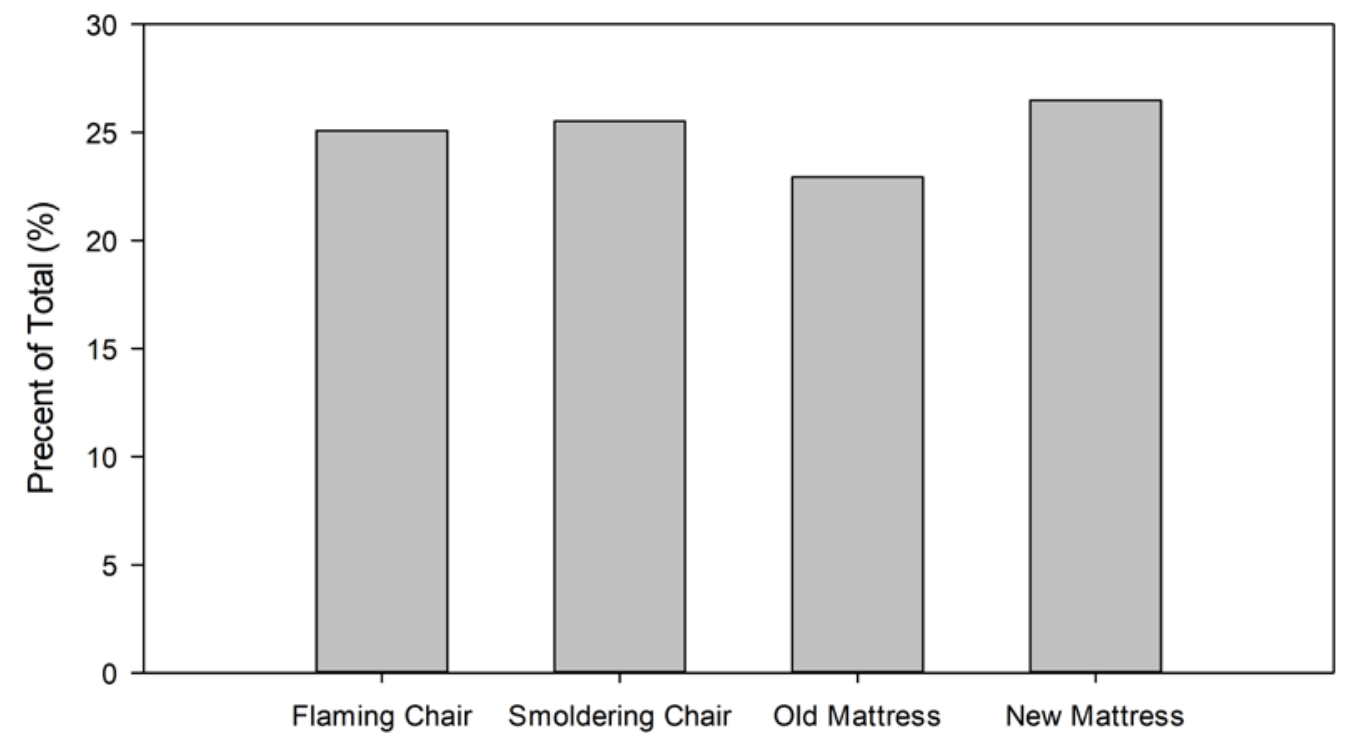

Figure 12 Histogram of percentage for each fire type.

One possibility for the discrepancies is that the simulations for the different fire types did not all fail at the same rate. Modifying the program to identify specifics of the failed cases, we ran an additional set of 1000 cases (Figure 13). The old mattress cases failed at a much higher rate than the other cases. It is likely due to the higher heat release rate of the old mattress category which provided a more challenging solution for the model. Note that the second largest percentage of failures is the flaming chair which is the second lowest percentage of total runs. The new mattresses have the highest percentage of runs and the lowest percentage of failures. The analysis shown in Figure 13 is consistent with the data in Figure 12 and seems to explain the discrepancies.

Fire detection time should depend on the proximity of the smoke alarm to the fire. In this study, smoke alarms were placed in a random room outside the room of fire origin. Figure 14 shows the number of doors between the fire room and the room with the alarm for the shortest path between the two rooms, for all the simulations. For example, if the fire room is connected to the room with the alarm, that case would be classified as one door. If there is a room in between the fire room and the room with the alarm, that case would be classified as two doors. Although not shown in the figure, there were 26 cases out of 57500 cases or $0.045 \%$ of the cases that had 5 links as the shortest path and 2 cases or $0.0034 \%$ of the cases where the shortest path was 6 links. There were no cases where the shortest path was longer than 6 links. 


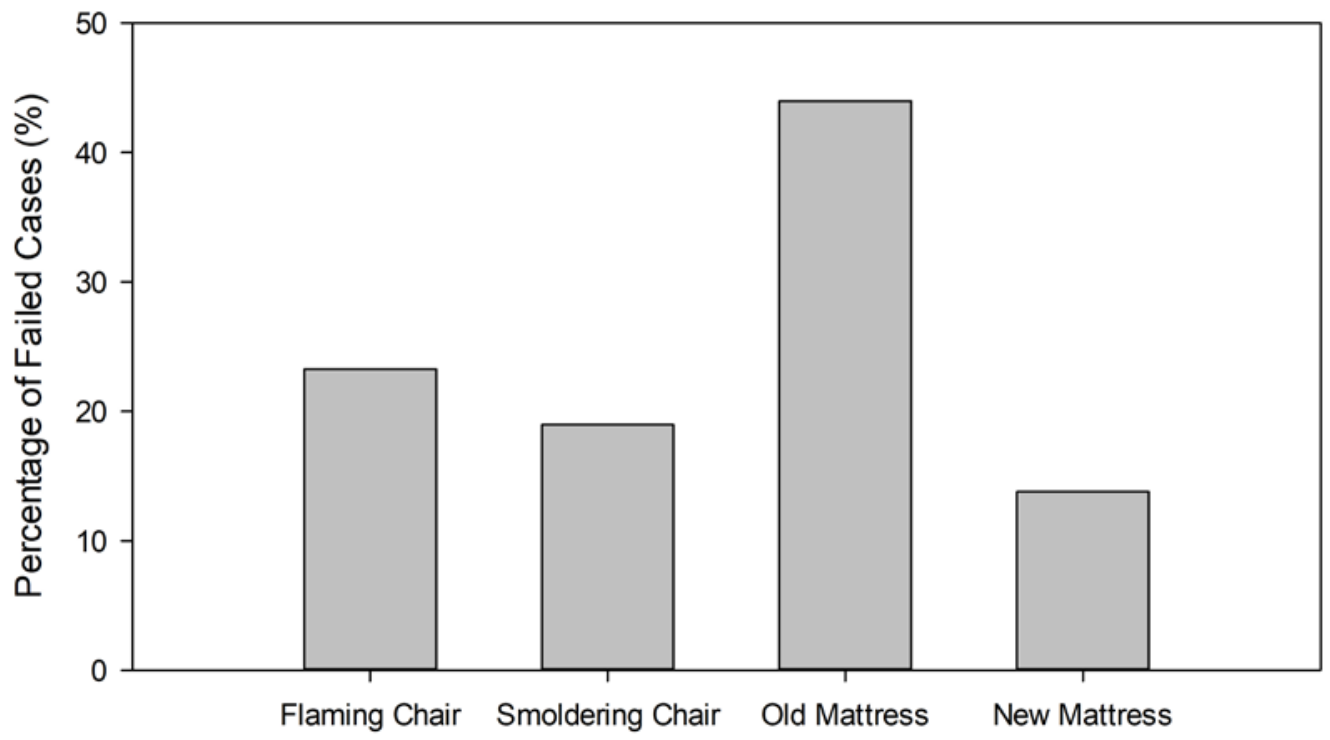

Figure 13 Histogram of percentage of failed cases by fire type.

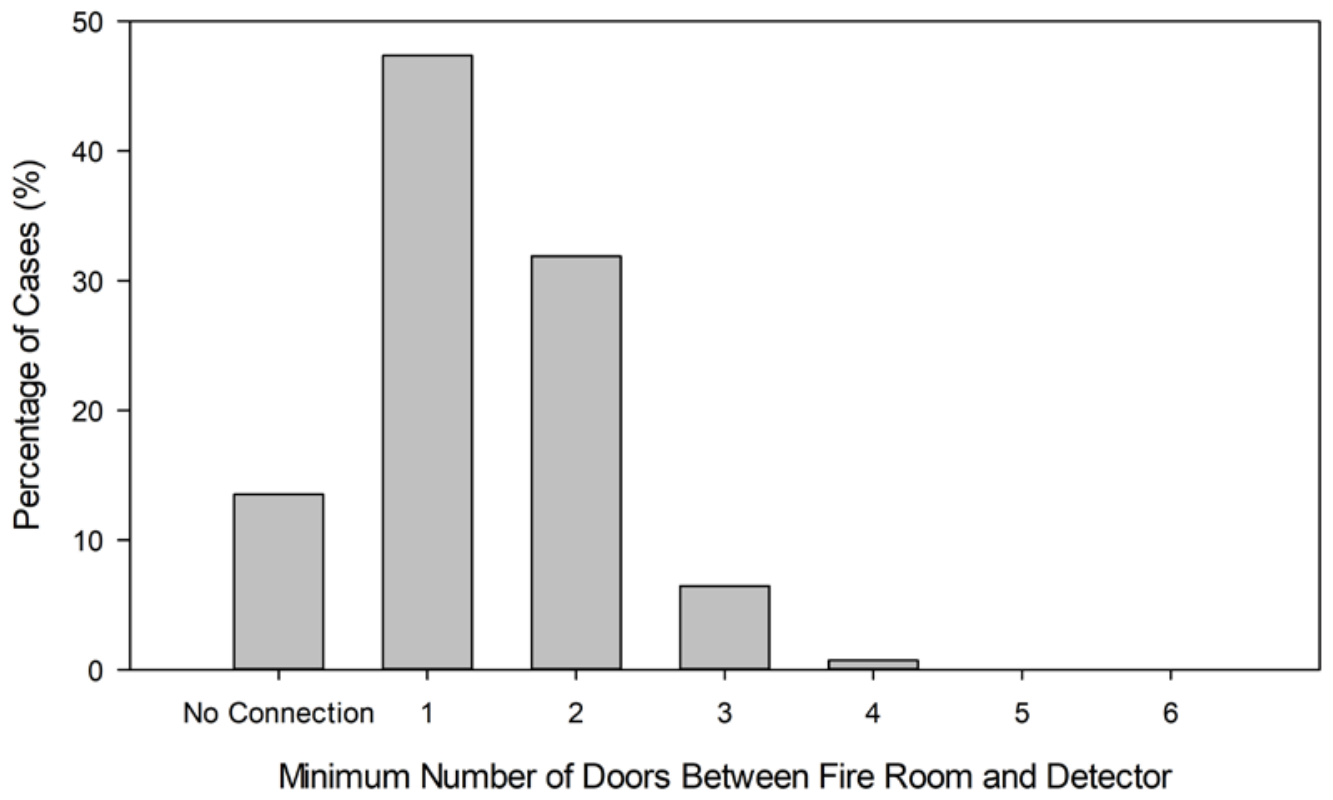

Figure 14 Histogram of percentage of cases with $n$ doors between the fire and smoke alarm.

Note that $13.5 \%$ of the cases do not have a path between the fire and the smoke alarm. In these cases, the alarm would not sound since these is no way for smoke from the fire to reach the alarm. Eliminating the cases that do not have a path between the fire and the alarm leaves 49725 cases. Considering only the cases with a path, the distribution of shortest path is shown in Figure 15. 


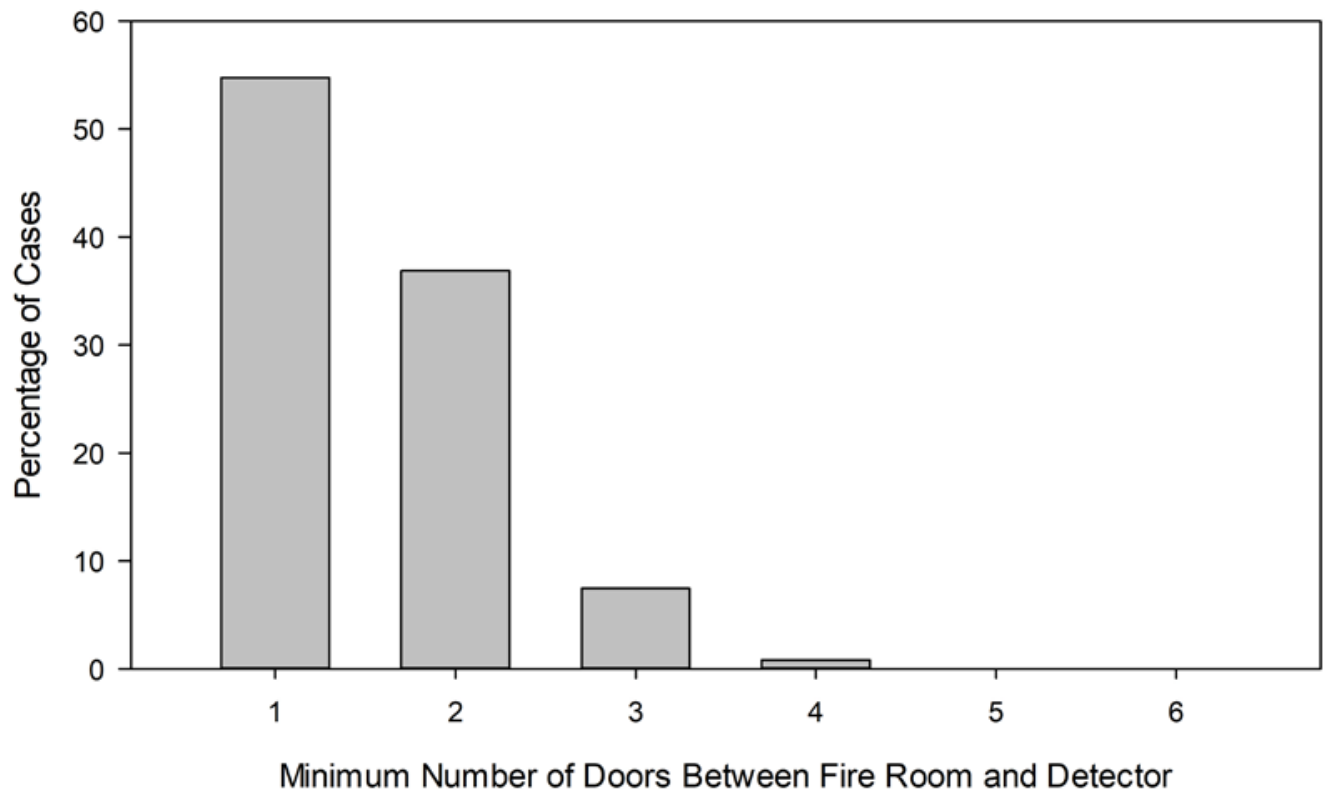

Figure 15 Histogram of percentage of cases with $\mathrm{n}$ doors between fire room and smoke alarm in cases with a path from fire to the alarm.

Of course, with the smaller set of 49725 cases, we need to ensure that there is not a bias introduced in the fire scenarios. Figure 16 shows that there is very little difference.

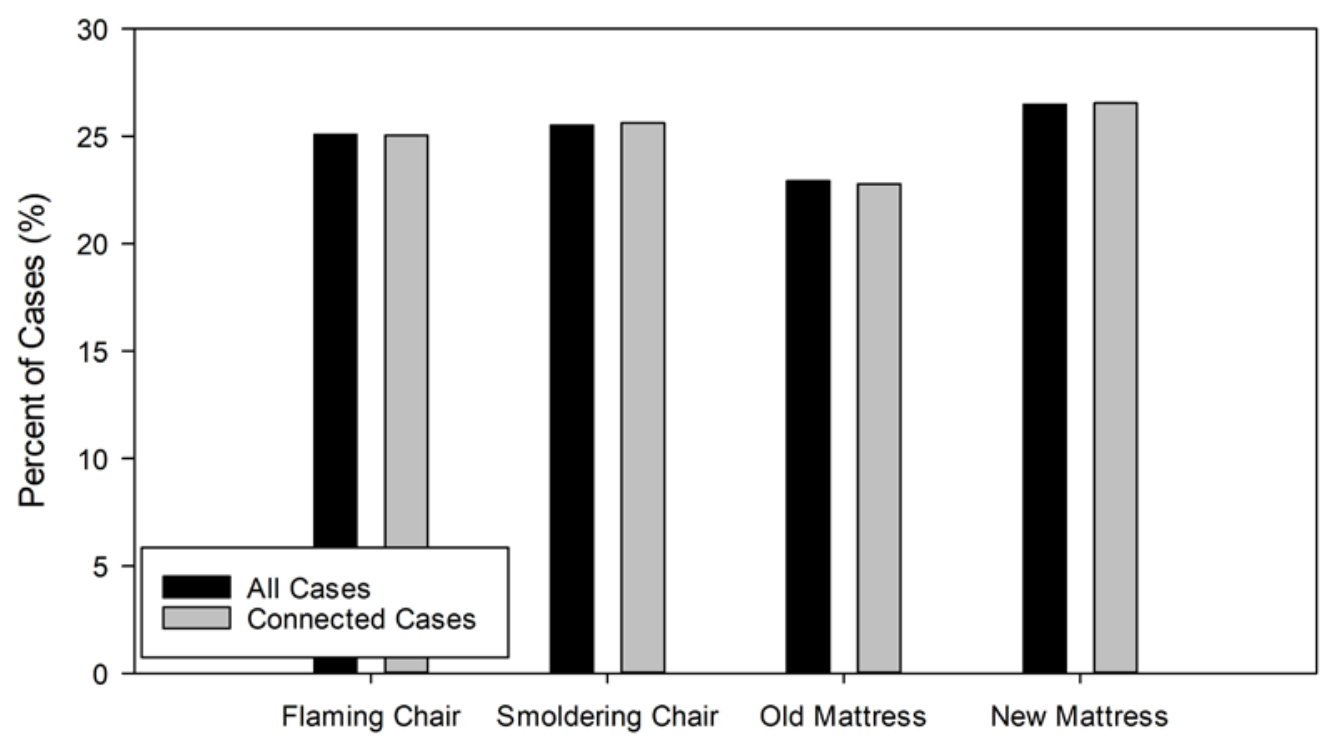

Figure 16 Histogram of percentage of cases with each fire type including all cases and only cases where the fire room is connected to the room containing a smoke alarm.

Finally, consider the distribution of rooms. Remembering that the distribution of rooms used to create the cases was based on the floor area of the structure, there was not a specific distribution for the number of rooms. Figure 17 shows the distribution of rooms for the total 
57500 cases that were run. Since the primary interest is in the impact of the new alarm technology in rooms remote from the fire, single room homes were not included although they are listed in the AHS.

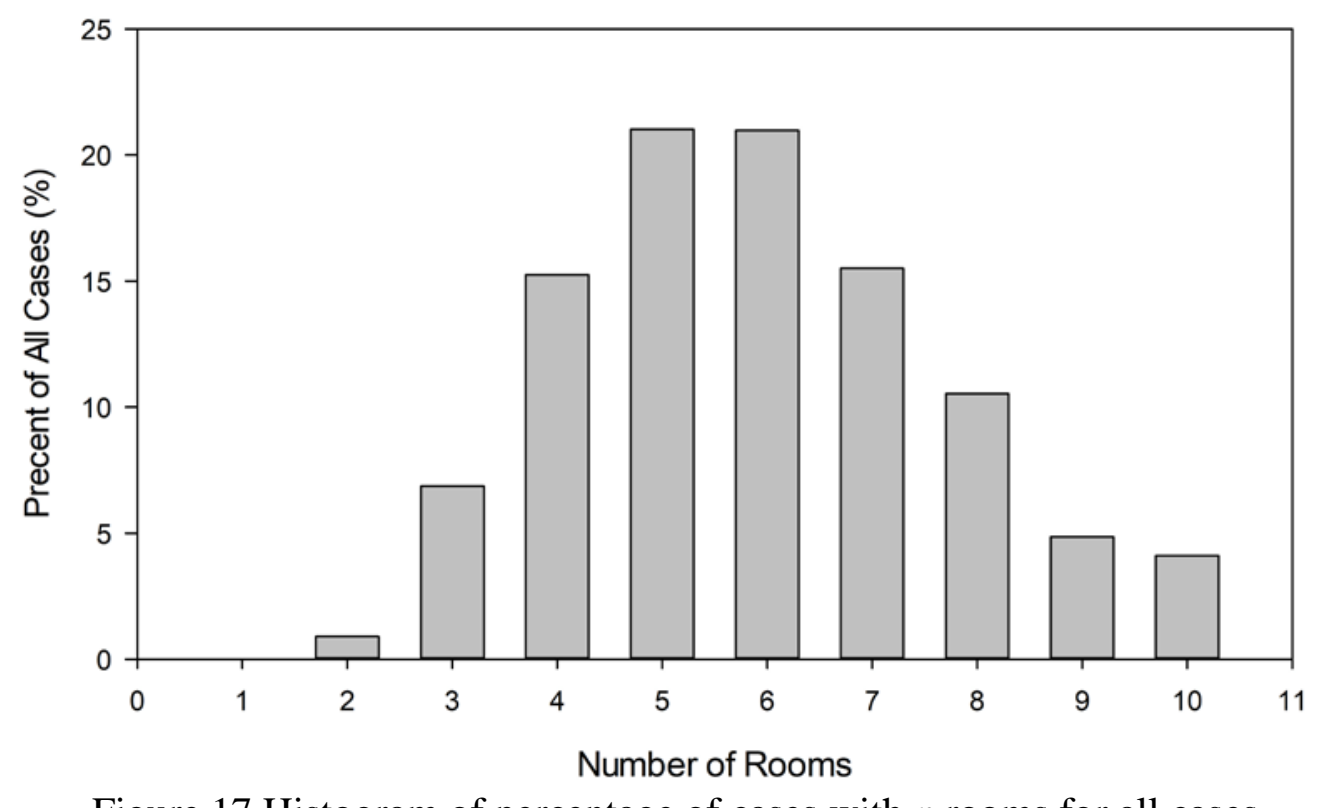

Figure 17 Histogram of percentage of cases with $n$ rooms for all cases.

Again, eliminating scenarios without a connection could introduce a bias in distribution of the number of rooms. Consider a two room house where there is only one possible path between the fire and the alarm compared to a ten room house which could have a number of different possible paths between the fire and the alarm. With a two room house, $50 \%$ of the cases would be expected to be removed if the presence of a connection was a random choice. One way to see this more clearly is instead of looking at the distribution of cases that have a path, look at the percentages of cases of each number of rooms that did not have a path.

Figure 18 shows that about half the two room case did not have a connection between the fire and the alarm whereas only about $1 \%$ of the ten room cases did not have a path. 


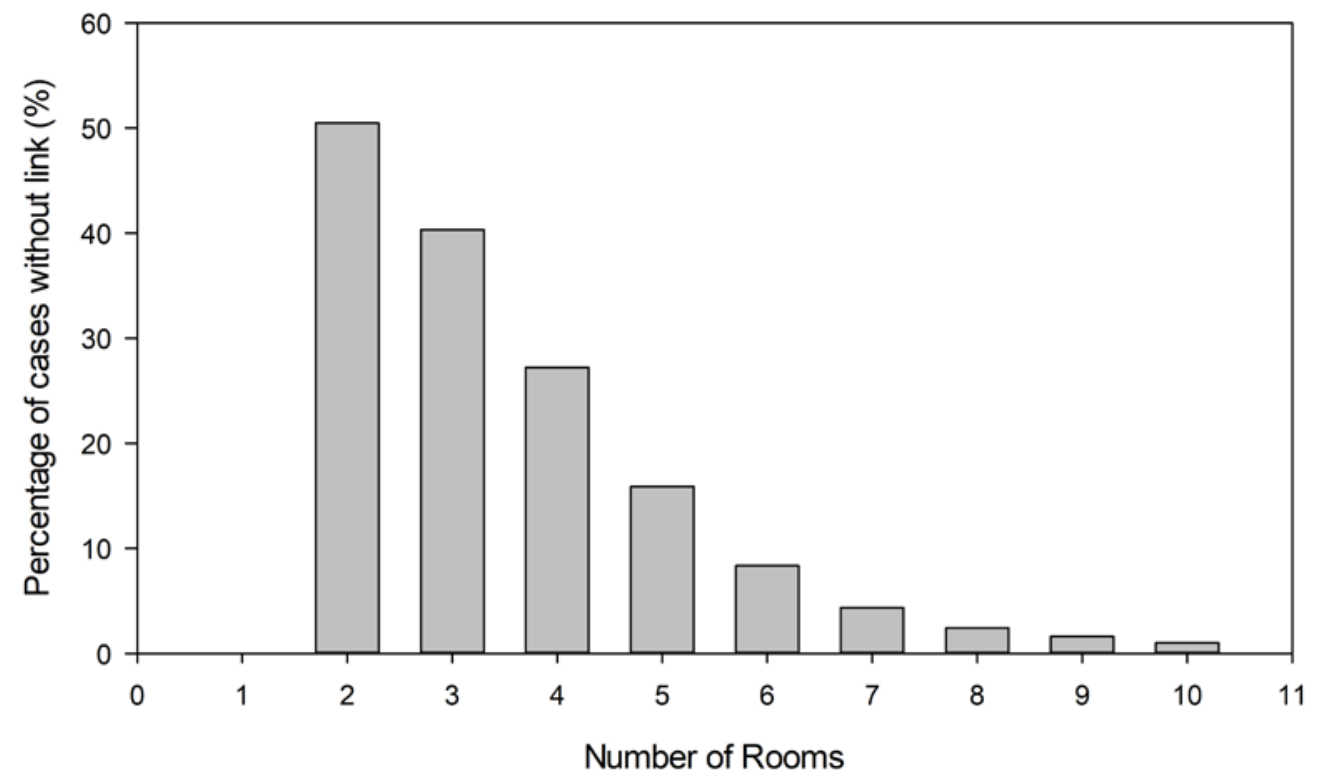

Figure 18 Percentage of cases with $n$ rooms where fire is not connected to a room with a smoke alarm.

To not have a biasing effect on the distribution of rooms, it would need to be the case that the percentage of cases without a connection would be about the same. Clearly that is not the case here. However, as can be seen in Figure 19 the bias in the distribution of rooms is not huge. The percentage of the cases with five rooms or fewer decrease some and the percentages of cases with six rooms or more increase some.

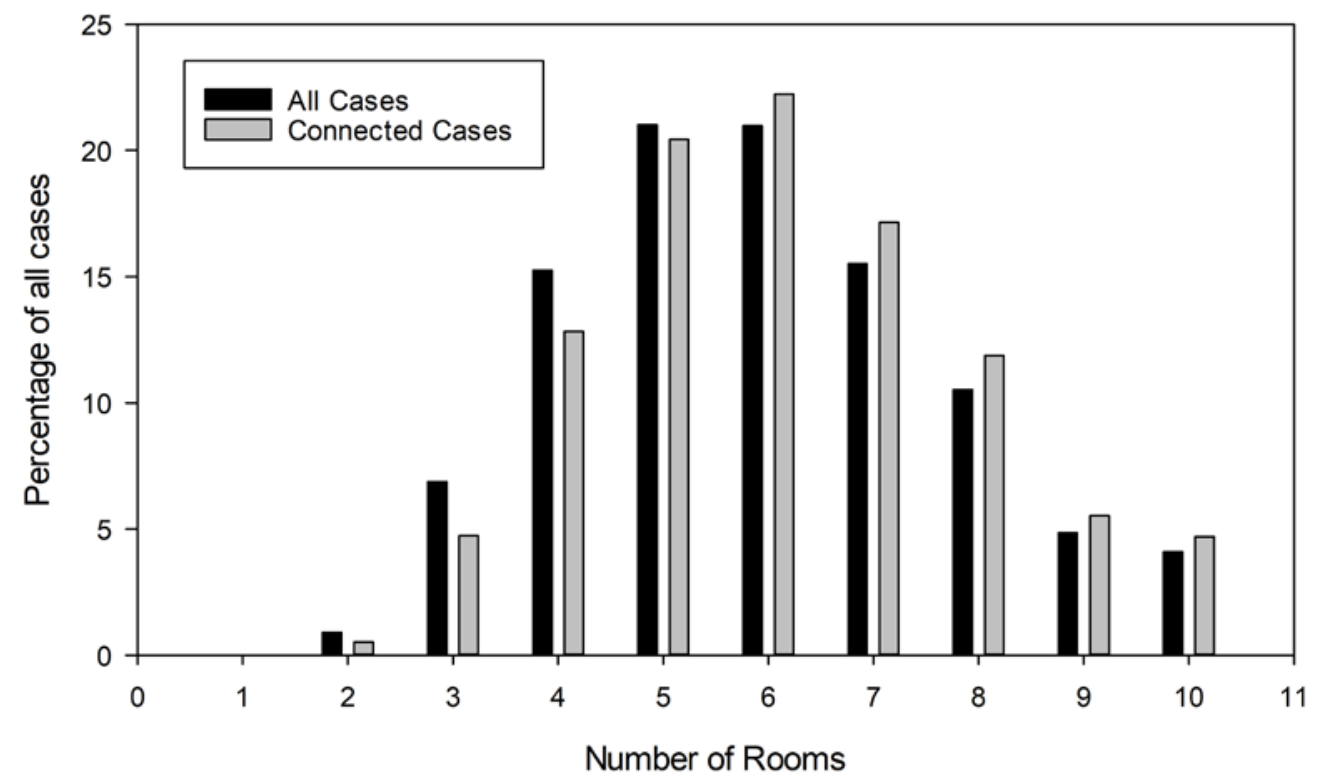

Figure 19 Comparison of the percentage of cases with $n$ rooms for all cases and only cases where fire is connected to a room with a smoke alarm. 
The question is whether the analysis would be impacted by this bias favoring homes with a larger number of rooms. In cases with a smaller number of rooms (which would be more likely to be excluded from the analysis than those with a larger number of rooms), there might be an impact increasing the detection time, but also one that reduces the evacuation time. Since our test analysis is to determine the impact of the new alarms and all cases of interest have alarms, it is not clear that it will have any real impact on the results. This still needs to be considered.

\subsection{How Much Data is Enough?}

One subject not touched on in any of the research reviewed in section 2.1 is how much modeling data is needed to draw valid conclusions. Rather, the approach taken was typically to run a specific number of cases assuming the results are statistically valid. In the past when computers were a lot slower, even CFAST runs could take significant computer time, so that running thousands or tens of thousands of cases was not practical.

With faster computers and newer software, it should be possible to run a lot more cases for the analysis. In order to better understand the process, it is important to understand the impact of running additional cases and when is to understand when sufficient cases have been run such that additional cases would not statistically alter the results of the analysis. For example, if it is practical to run only ten thousand runs, but twenty thousand runs are needed to confidently understand what the model is predicting, the method being used may not be appropriate to quantitatively understand the impact of new fire technology.

For some statistics of interest, there are well known and easily calculated measures of uncertainty. For example, in statistics, the mean of a group of random variables is also a random variable with a normal distribution and a standard deviation of

$$
\frac{\sigma}{\sqrt{n}}
$$

where $n$ is the number of values in the average and $\sigma$ is the standard deviation of the set of values being averaged. One average value we calculate is the average difference in alarm times between ionization alarms and the new alarms for new mattress fires. For 13197 new mattress fires, the mean difference in alarm times is $49.37 \mathrm{~s} \pm 0.88 \mathrm{~s}$. Since the mean difference is being used rather than a pass/fail criterion, there is not an obvious required level of precision. Still, we do know this average to a fairly precise range, with a standard deviation of the mean less than $1 \mathrm{~s}$.

For more complicated comparisons, consider a histogram of the distribution of the differences in ionization alarms and new alarms. One way to determine if there is enough data is to plot the statistics of interest with increasingly larger random samples of the data. When the calculated values converge, there is little value in running additional cases. 


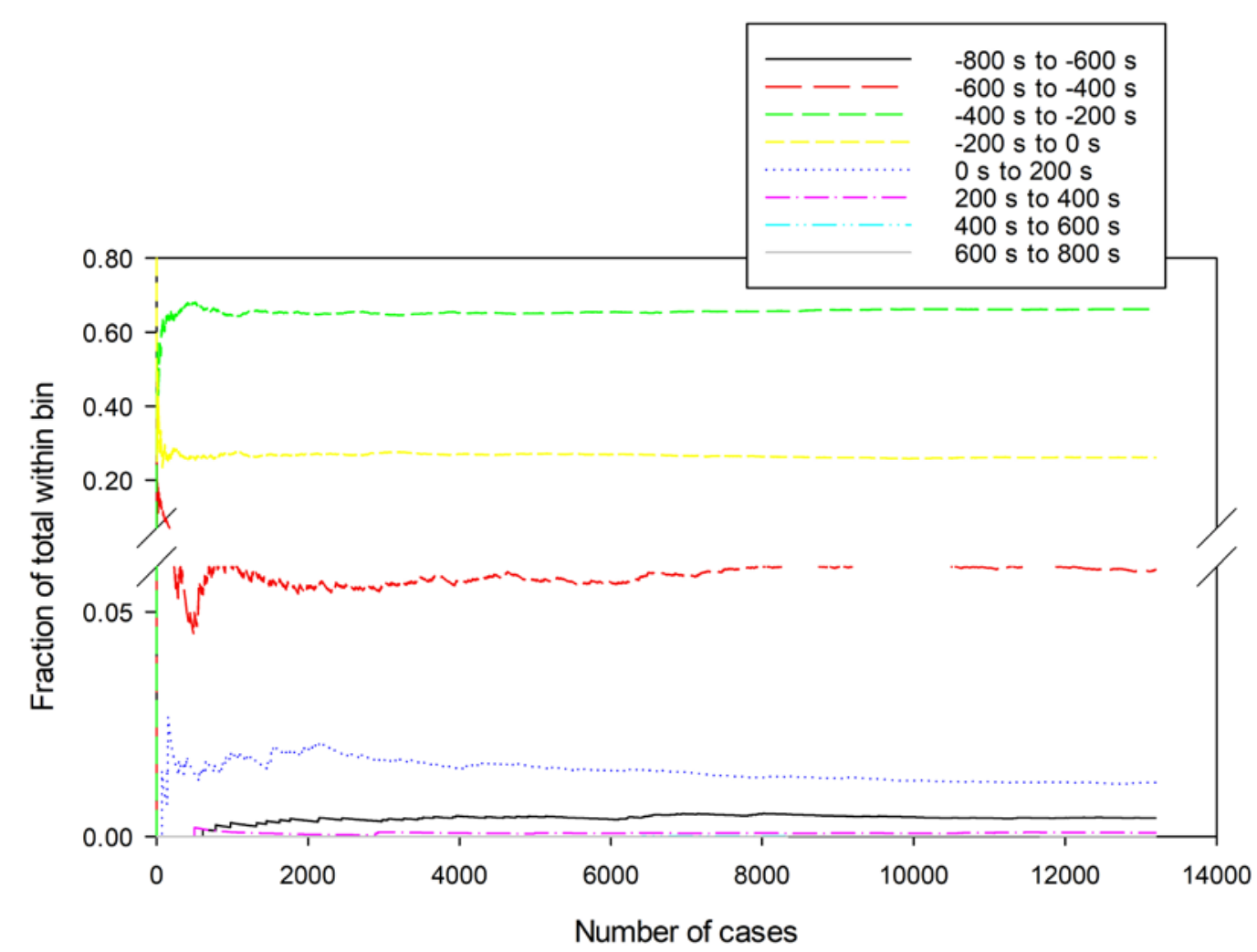

Figure 20 Bin values for histogram of the calculated detection time differences between ionization and new alarms for new mattress fires.

Figure 20 shows the fraction of new mattress fire cases falling into a particular range of detection time difference in a histogram as a function of the total number of cases run. For example, the top curve in Figure 20 is the fraction of all connected cases run to that point with the new mattress fires where the ionization alarm goes off between $400 \mathrm{~s}$ and $200 \mathrm{~s}$ before the new alarm activates. While there is some movement of bin values, especially for less than 1000 cases, the values settle down significantly with larger samples. However, the lines are not perfectly flat even after 10000 cases. Note that many of the bins have fractional values very close to zero.

Another method to determine the number of runs needed is to perform a bootstrap analysis using increasingly large samples from the data. For this analysis we used 250 to 2500 (in increments of 250) data points from the model results (drawn randomly). For each number of data points, the analysis was run 1000 times, each time with a different randomly drawn set for analysis. The coefficient of variation (i.e., standard deviation divided by the mean value) on selected estimated parameters were computed. A graph of the coefficient of variation for estimated activation times versus number of data points for the flaming chair scenarios is shown in Figure 21. Shown are the coefficients of variation for the ASET time for ionization and new alarms using an FED of 0.5 ("ion.ASET0.5" and "new.ASET0.5”) and the estimated variance in the values for ionization and new alarms ("var.ion" and "var.new"). Other parameters and fire types are similar. Above about 1000 simulations, the coefficient of variation is fairly constant; below 1000, larger values for the coefficient of variation are seen. 


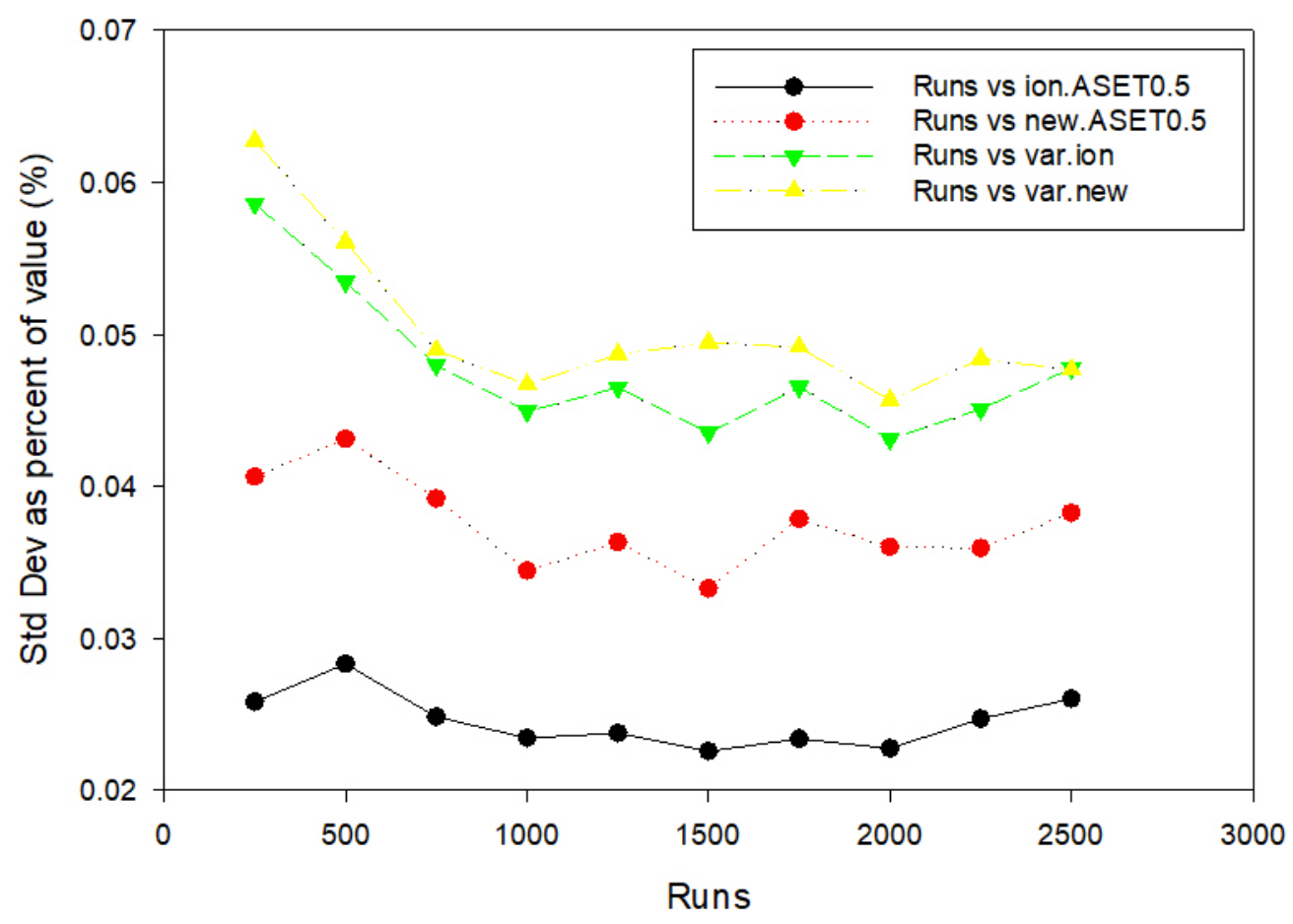

Figure 21 Showing the absolute value of the coefficient of variation versus number of bootstrap runs for values calculated for the flaming chair fires.

For our analysis of these measures of performance, it is certainly the case that we could have taken less than half the cases and probably only a quarter of the data we generated. For other analyses this might not be true. For each analysis the number needed to obtain consistent statistics can vary significantly.

\subsection{Smoke Alarm Activation}

We start the analysis by looking at the smoke alarm activation. From the parameters that we are using, there are clear expectations in how the alarms will activate relative to each other. If the relative performance is as expected, it will increase confidence in the results of the occupant safety analysis. If there are differences from the expected performance, it is important to understand those differences before moving on with the analysis.

First, consider the difference between the new alarm and the ionization alarm. A starting point is to look at simple statistics for each of the fire types. Starting with the flaming chair, there are 12457 cases. The differences in alarm activation times range from -695 s to $618 \mathrm{~s}$. To be clear there is at least one case where the new alarm activated $695 \mathrm{~s}$ before the ionization alarm and at least one case where the new alarm activated $618 \mathrm{~s}$ after the ionization alarm. The average difference is $46.23 \mathrm{~s} \pm 91.6 \mathrm{~s}$. The definition of the standard deviation of the sample mean is given in Eq. (3). The basic statistics for all three flaming fire types and the combined flaming fire are in Table 4. 
Table 4 Basic statistics for new alarm activation times minus ionization alarm activation times for flaming fires. Uncertainty is shown as a single sample standard deviation.

\begin{tabular}{|c|c|c|c|l|}
\hline & Total & Min (s) & Max (s) & \multicolumn{1}{|c|}{ Mean (s) } \\
\hline Chair & 12457 & -695 & 618 & $46.23 \pm 91.6$ \\
\hline Old Mattress & 11326 & -475 & 647 & $45.07 \pm 90.1$ \\
\hline New Mattress & 13197 & -996 & 715 & $49.39 \pm 101.4$ \\
\hline All Flaming Fires & 36980 & -996 & 715 & $47.00 \pm 94.8$ \\
\hline
\end{tabular}

The new alarm compared to ionization alarm statistics for the smoldering fire are very different than for flaming fires. There are 12740 cases with a range of $-5070 \mathrm{~s}$ to $1930 \mathrm{~s}$. The average is $-1128.74 \mathrm{~s} \pm 649.2 \mathrm{~s}$.

On average, the ionization alarms activate a little before the new alarms in flaming fires, but the new alarms activate a faster than the ionization alarms in the smoldering fires. As discussed in section 4.4, this trend can be misleading for the overall performance because smoldering fires are assumed to be so much slower in developing. Most of the time when the ionization alarm activates, there is still sufficient time for occupants to safely evacuate. So, the average does not always provide a sufficient description of overall performance.

Figure 22 shows the distribution of the activation time for the three types of flaming fires as well as all flaming fires combined. Even though we have more than 10000 cases for each fire type, there are still some differences in the distributions of the results depending on the fire type. The differences are not enough to invalidate grouping the three fires as one fire type, but depending on the issue of interest, it might be important to understand why the distributions have the differences they do. 


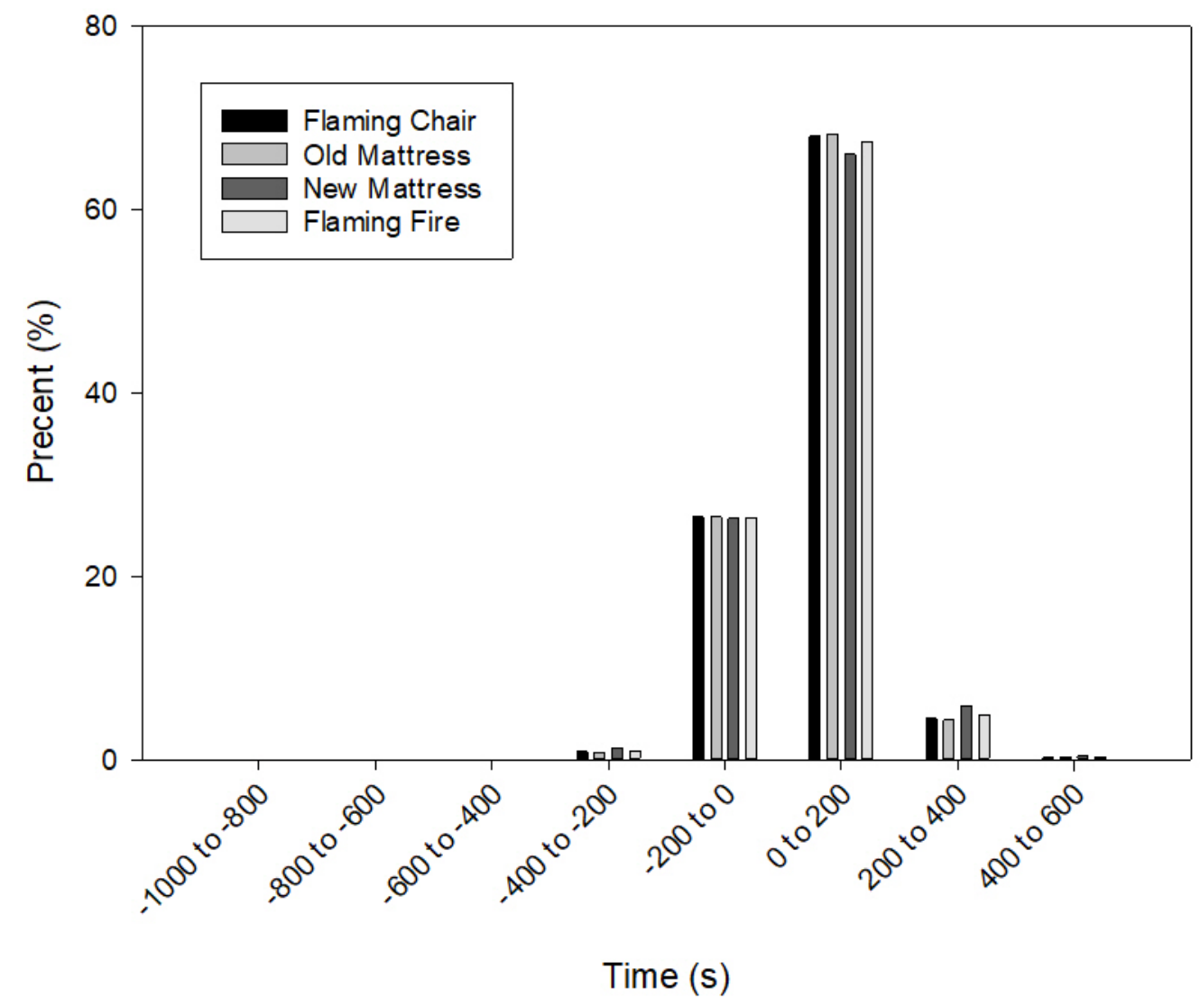

Figure 22 Distribution of differences in new minus ionization alarm activation time for flaming fires.

In Figure 23 zooms in on $0 \%$ to $3 \%$ of Figure 22 to be able to view the data in the outer bins. At this scale, the percent of cases that end up in the -1000 to -800 bin are very small, only $0.01 \%$. While it is not a significant part of the distribution, all bins that have at least one case are shown in the histograms to represent the range of the differences in any two alarms' responses. 


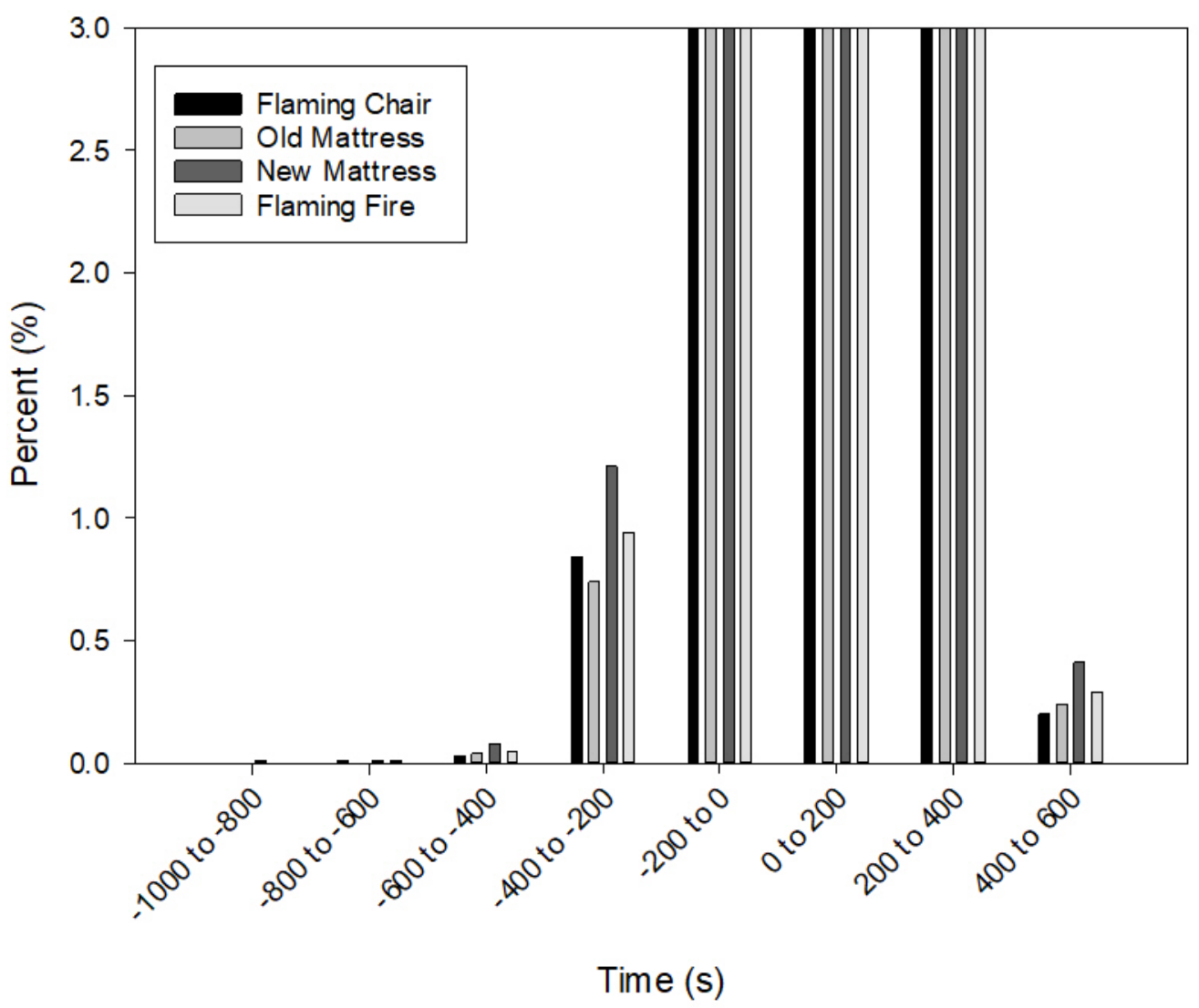

Figure 23 Close up of distribution of differences in new minus ionization alarm activation time for flaming fires.

The distribution of the activation times for the new alarms minus ionization alarms for the smoldering chair must be put on a different chart because the times are on such a different scale, as expected from the averages of the difference. Figure 24 shows the distribution of the difference in activation time of new alarms and ionization alarms. Notice the difference in the distributions in Figure 22 and Figure 24. While the three flaming fires are skewed a little to the left, the smoldering fires are skewed more drastically to the left. In fact, just a little over $13 \%$ of the cases are to the right of the largest bin (from $-1000 \mathrm{~s}$ to $-500 \mathrm{~s}$ ). 


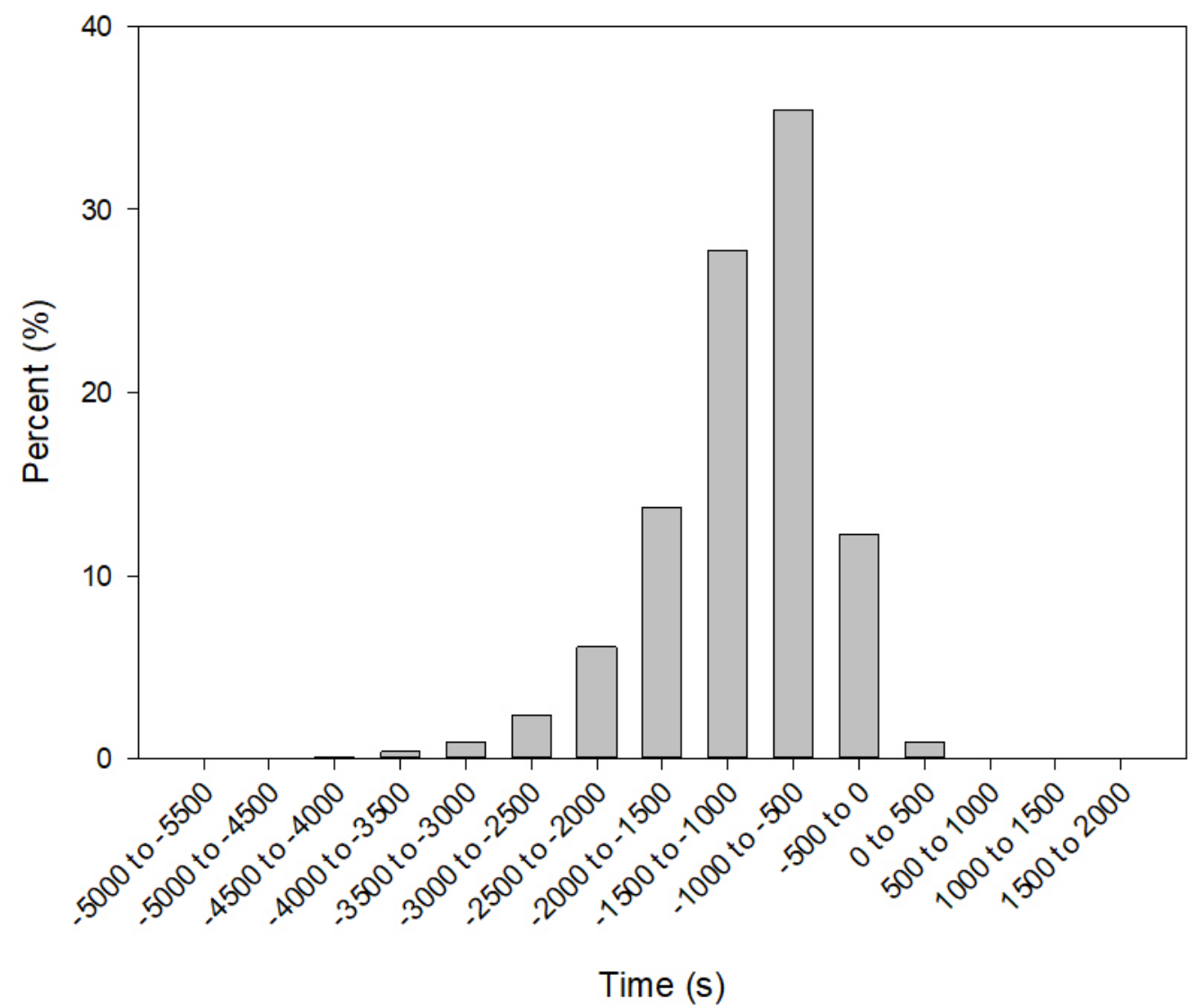

Figure 24 Distribution of difference in new and ionization alarm activation time for smoldering chair.

Now look at the differences in the activation times between the new alarms and the photoelectric alarms. First is the table of basic statistics.

Table 5 Basic statistics for new alarm activation times minus photoelectric alarm activation times for flaming fires. Uncertainty is shown as a single sample standard deviation.

\begin{tabular}{|c|c|c|c|c|c|}
\hline & Total & Min (s) & Max (s) & \multicolumn{2}{c|}{ Mean (s) } \\
\hline Chair & 12451 & -835 & 425 & $-146.88 \pm$ & 100.8 \\
\hline Old Mattress & 11326 & -807 & 311 & $-141.13 \pm$ & 100.3 \\
\hline New Mattress & 13195 & -840 & 381 & $-169.28 \pm$ & 106.1 \\
\hline All Flaming Fires & 36972 & -840 & 425 & $-153.11 \pm$ & 103.3 \\
\hline
\end{tabular}

Note that the differences for the new mattress fires and the other fire types are a little more pronounced. However, the response times, as seen in section 4.4, are close enough to be considered one fire type for our purposes.

For the smoldering chair fires, the differences between new and photoelectric alarms ranges from $-3690 \mathrm{~s}$ to $5370 \mathrm{~s}$. The average difference is $441.54 \mathrm{~s} \pm 665.5 \mathrm{~s}$. 
For the flaming fires the new alarms activate an average of more than 2.5 min ahead of the photoelectric alarms, but an average of more than 7.3 minutes after the photoelectric alarms for the smoldering fires.

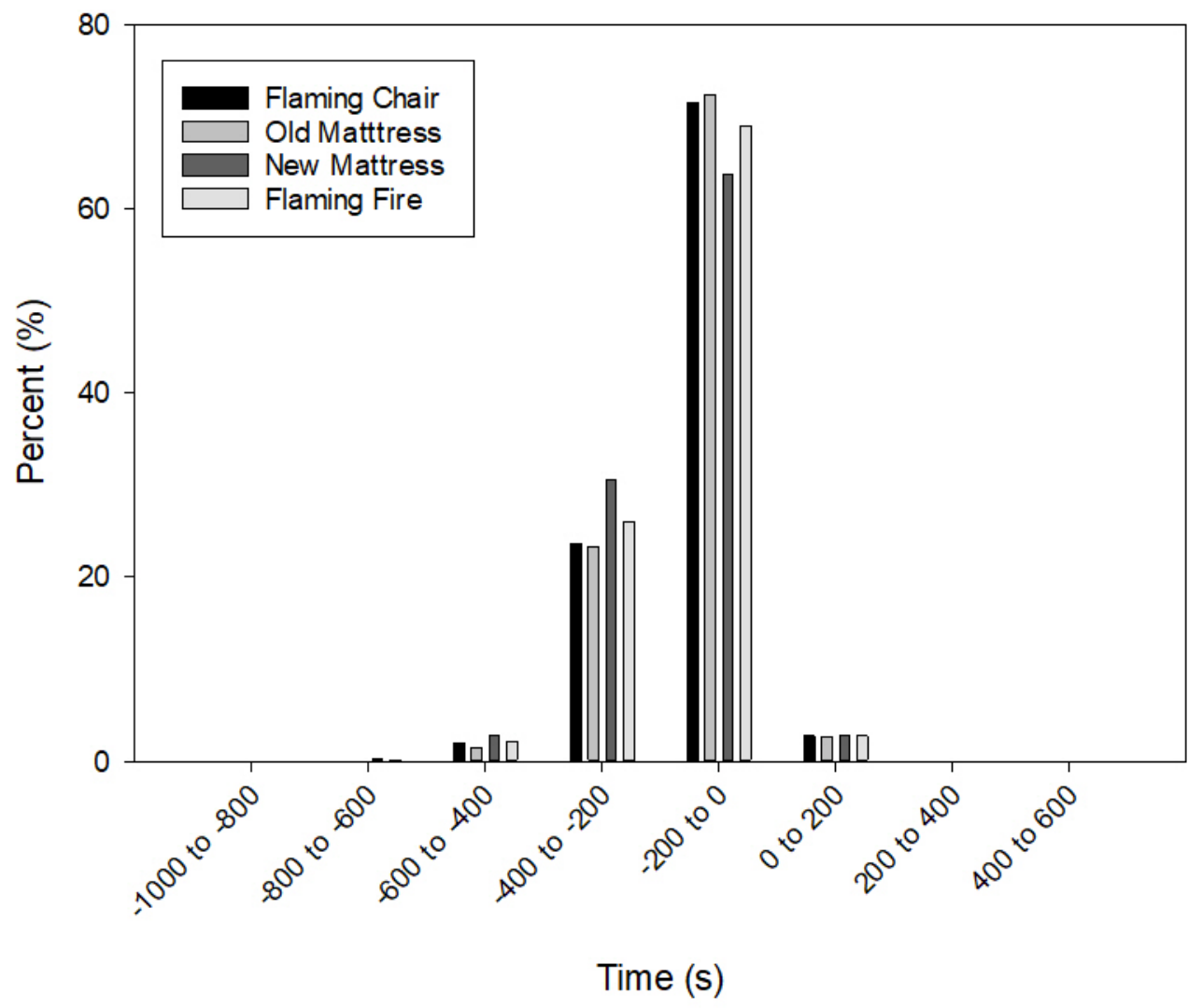

Figure 25 Distribution of differences in new minus photoelectric alarm activation time for flaming fires.

As can be seen Figure 25 is very similar to Figure 22 just shifted to the left one bin. This is not surprising since the new alarms are assumed to be slightly less sensitive than the ionization alarms but more sensitive than the photoelectric alarms to flaming smoke. The reason the data for the new mattress fires are a little more spread out than the other two is not known but for this analysis it does not appear to be of importance. 


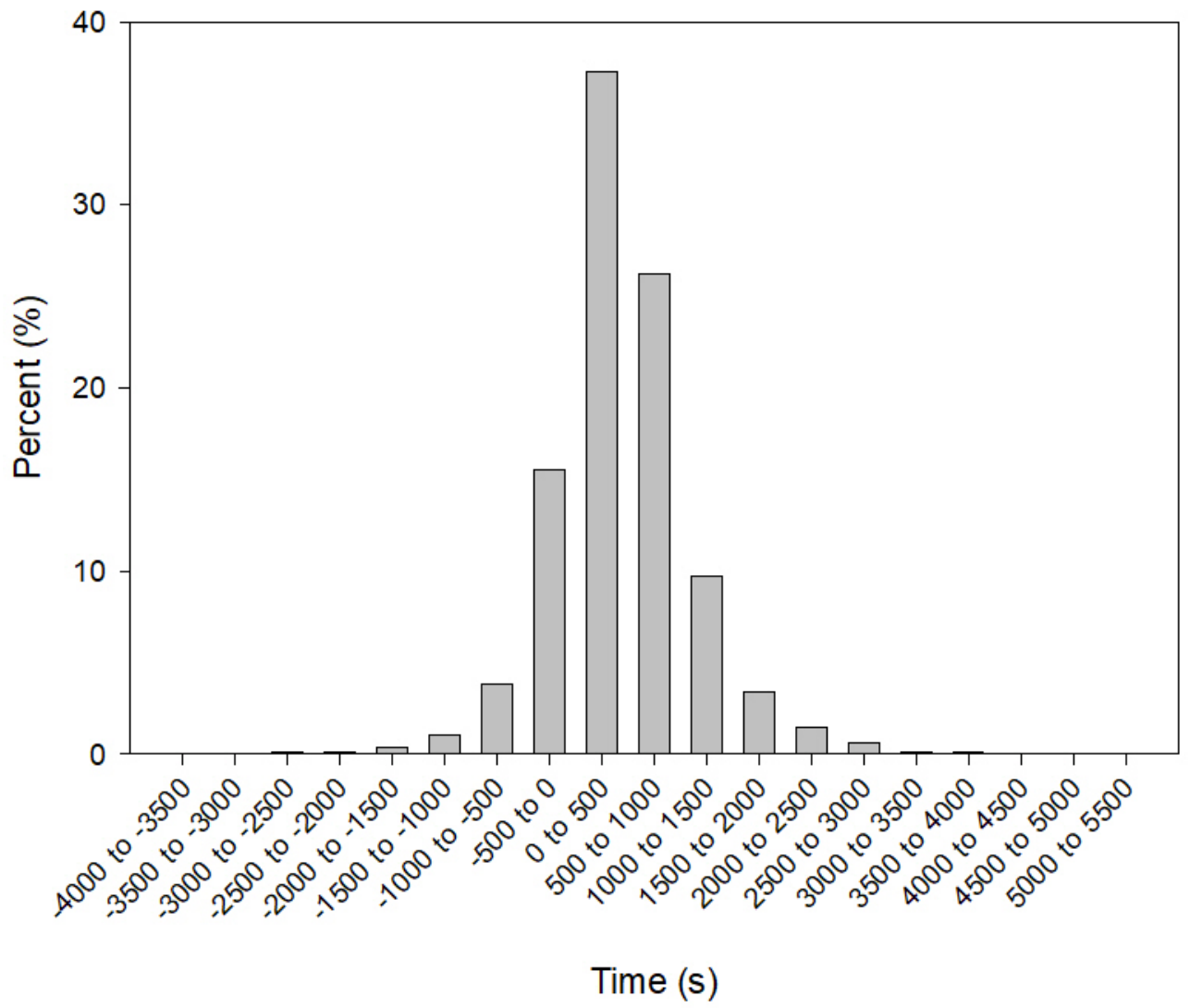

Figure 26 Distribution of differences in new minus photoelectric alarm activation time for smoldering fires.

Unlike with the flaming fires where the distributions of the new alarms minus the ionization alarms were just shifted from the difference between the new and photoelectric alarms, the smoldering fires behaves differently between the ionization and photoelectric alarms compared to the new alarm, Figure 26 shows a much more symmetric distribution compared to the one in Figure 24 for the ion photoelectric alarms. This is probably because the assumed smoke obscuration at activation of the new alarms are closer to those of current photoelectric alarms than they are to current ionization alarms in the presence of smoldering smoke.

One final way to look at the alarm activation is to realize that, since the alarms are reacting to the same environment, it should be possible to model one alarm using another. For example, we can find the relationship between the new alarm and the ionization alarm, such that for a given ionization alarm activation time, we can estimate when the new alarm will activate on average.

First, we estimate the multivariate normal parameters for the data. Assume activation times $t$ for the alarms are jointly multivariate normal, that is,

$$
t \sim N(\mu, \Sigma)
$$


where $\mu$ is the vector of mean values, and $\Sigma$ is the covariance matrix. We estimate $\mu$ and $\Sigma$. The vast majority of the variance is explained by a single variable, which is the principal eigenvector for the covariance matrix.

We are interested in identifying the differences in activation times between the different alarms. Specifically, we estimate the conditional probability distribution of

$$
t_{i}-t_{j} \mid u
$$

where $u=c v_{1} \cdot t$ for some normalzing constant, $c$, and where the principal eigenvector of the estimated covariance matrix is $v_{1}$. Here, $u$ can be thought of as a standardized activation time, and in effect is approximately the mean activation time.

The analysis was conducted in STAN [61].

We ran two versions of this model for flaming fires. In one version, separate parameter estimates were made for each different type of flaming fire. A second version was run where the fire types were combined, and a single set of parameters was estimated. The likelihood ratio test indicates that the probability of the different fire types having an identical relationship is less than $1 \times 10^{-16}$, which indicates that the relationships are not identical across different fire types. Results for the first set of estimates are included in Table 6. 
Table 6 Slope and intercept for differences in activation times for different types of alarms.

\begin{tabular}{|c|c|c|c|c|c|}
\hline Fire type $^{\text {a }}$ & type & estimated value & t-statistic & p.value ${ }^{\text {b }}$ & code $^{c}$ \\
\hline \multicolumn{6}{|c|}{ ion-photo } \\
\hline chair & slope & $-0.13 \pm 0.0075$ & -17.0 & 0.0000 & $* * *$ \\
\hline new mattress & slope & $-0.13 \pm 0.0069$ & -18.5 & 0.0000 & $* * *$ \\
\hline old mattress & slope & $-0.098 \pm 0.0076$ & -13.0 & 0.0000 & $* * *$ \\
\hline chair (smoldering) & slope & $0.13 \pm 0.0043$ & 30.5 & 0.0000 & $* * *$ \\
\hline chair & intercept & $-82.3 \pm 6.9$ & -11.9 & 0.0000 & $* * *$ \\
\hline new mattress & intercept & $-98.8 \pm 6.7$ & -14.7 & 0.0000 & $* * *$ \\
\hline old mattress & intercept & $-109 . \pm 7.2$ & -15.3 & 0.0000 & $* * *$ \\
\hline chair (smoldering) & intercept & $636 . \pm 33.1$ & 19.2 & 0.0000 & $* * *$ \\
\hline
\end{tabular}

new-ion

\begin{tabular}{|l|l|c|c|r|l|}
\hline chair & slope & $0.048 \pm 0.0058$ & 8.3 & 0.0000 & $* * *$ \\
\hline new mattress & slope & $0.033 \pm 0.0060$ & 5.5 & 0.0000 & $* * *$ \\
\hline old mattress & slope & $0.020 \pm 0.0062$ & 3.2 & 0.0012 & $* *$ \\
\hline chair (smoldering) & slope & $-0.084 \pm 0.0034$ & -24.7 & 0.0000 & $* * *$ \\
\hline chair & intercept & $8.3 \pm 5.4$ & 1.5 & 0.13 & \\
\hline new mattress & intercept & $17.7 \pm 5.9$ & 3.0 & 0.0025 & $* *$ \\
\hline old mattress & intercept & $28.0 \pm 5.9$ & 4.8 & 0.0000 & $* * *$ \\
\hline chair (smoldering) & intercept & $-532 . \pm 26.3$ & -20.3 & 0.0000 & $* * *$ \\
\hline
\end{tabular}

New-photo

\begin{tabular}{|l|l|l|r|r|l|}
\hline chair & slope & $-0.080 \pm 0.0069$ & -11.5 & 0.0000 & $* * *$ \\
\hline new mattress & slope & $-0.096 \pm 0.0061$ & -15.8 & 0.0000 & $* * *$ \\
\hline old mattress & slope & $-0.078 \pm 0.0066$ & -11.9 & 0.0000 & $* * *$ \\
\hline chair (smoldering) & slope & $0.048 \pm 0.0039$ & 12.3 & 0.0000 & $* * *$ \\
\hline chair & intercept & $-74.0 \pm 6.4$ & -11.6 & 0.0000 & $* * *$ \\
\hline new mattress & intercept & $-81.1 \pm 5.9$ & -13.7 & 0.0000 & $* * *$ \\
\hline old mattress & intercept & $-81.3 \pm 6.2$ & -13.1 & 0.0000 & $* * *$ \\
\hline chair (smoldering) & intercept & $104.1 \pm 30.1$ & 3.5 & 0.0005 & $* * *$ \\
\hline
\end{tabular}

a - Unless noted, chair, old mattress and new mattress are flaming ignitions.

b - 'P.value' is the two-sided error probability for the estimate. 'Code' is '***' if the p value $<0.001$, '**' if the $\mathrm{p}$ value $<0.01$, '*' if the $\mathrm{p}$ value $<0.05$, and '.' if the $\mathrm{p}$ value $<0.1$.

Much of the variance in the data is explained by the principal eigenvector $(u)$ of the covariance matrix, which can be thought of as an index of the severity of the fire. Table 7, below shows how much of the variance in the activation times is explained by the $u$ index for each fire type in the r-squared column. What that means is that once the severity of the fire is 
known (in the sense of knowing the value of the $u$ index) then $80 \%$ to $90 \%$ of the alarm activation times are known.

Table 7 Coefficient of variation by fire type showing how much variance is explained by $u$ index

\begin{tabular}{|c|c|c|}
\hline Fire Type & r-squared & Std. Error \\
\hline Chair (flaming) & 0.8066 & 0.0056 \\
\hline Chair (smoldering) & 0.9122 & 0.0028 \\
\hline Old Mattress (flaming) & 0.8049 & 0.0055 \\
\hline New Mattress (flaming) & 0.8198 & 0.0053 \\
\hline
\end{tabular}

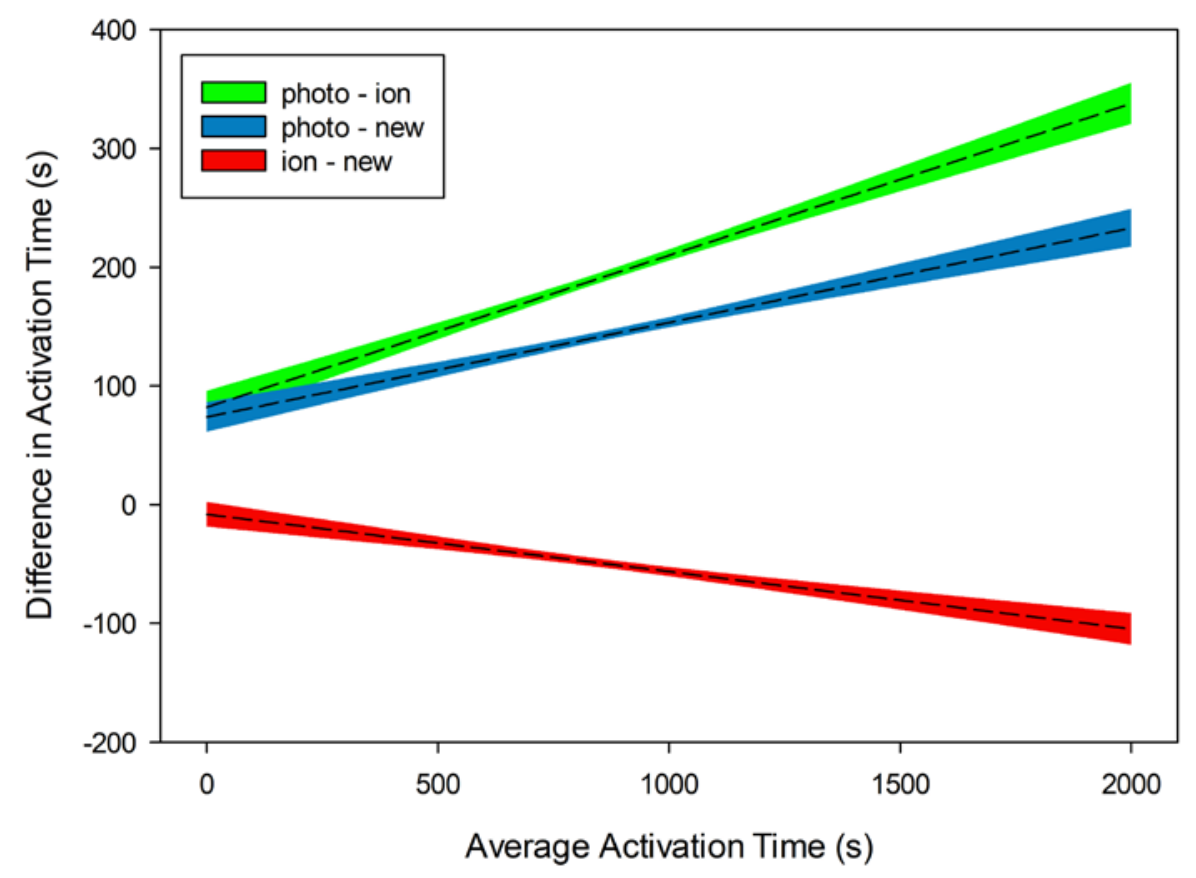

Figure 27 For flaming chair, difference and uncertainty in activation times based on average activation time. 


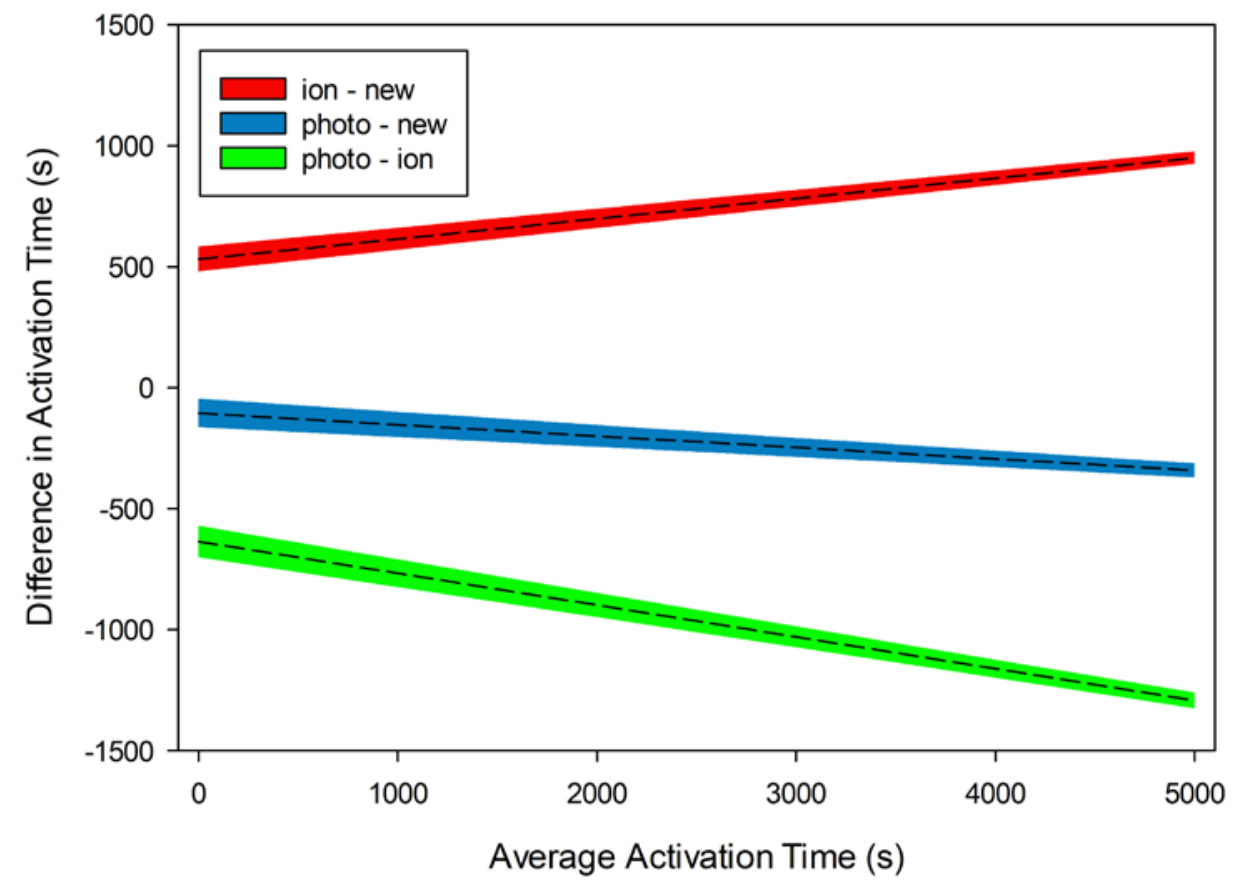

Figure 28 For smoldering chair, difference and uncertainty in activation times based on average activation time.

Figure 27 and Figure 28 show all the differences between the three alarms as a function of the average activation times for the pair of detectors for two fire types: flaming chair and smoldering chair. To be clear in Figure 28 for the ion - new difference, the x-axis is the average activation time of the ion and new detectors, however, for the photo - new the x-axis is the average activation time of the photo and new detectors. Keep in mind that this is modeling the differences between any two alarm types so a line with positive values indicates that the first alarm is slower than the second alarm, and negative values means the first alarm is faster than the second.

From the flaming chair in Figure 27, we see that the ionization alarm is faster than the new alarm and that difference increases as the average time for the alarms to activate increases. Both the ionization and new alarms are faster than the photoelectric alarms with the difference being the greatest for the photoelectric - ionization time. The other two flaming fires are similar though not identical. These results are consistent with how the alarms are expected to respond.

The increasing time difference as the average time increases makes sense because the alarms are activating in the early part of the fire, when fire growth is assumed to be a linear ramp. For example, consider the average smoke optical density for the ionization and new alarms activations. If the smoke's optical density is increasing quickly then it will trigger the ionization alarm quickly, but it will also trigger the new alarm quickly, and so the difference between the ionization and new alarm activations will not be that great. In a case where 
everything is happening significantly slower, the ionization alarm will activate much later, but the time for the smoke to trigger the new alarm will also be longer, and the difference between the two will be greater.

The results for the smoldering chair, in Figure 28, look quite different. For smoldering fires, the photoelectric alarm is faster than either the ionization or the new alarms, which is again as expected. Also, the new alarm is faster than the ionization alarm in smoldering fires, which is part of the intention. Note how much faster the photoelectric alarm is in comparison to the ionization alarm than it is to the new alarm. Again, this is part of the intention of the requirements for the new alarms.

\subsection{Occupant Safety}

Occupant safety can be achieved in either of two ways: to leave the building before conditions become untenable or to put the fire out without being injured. While nobody would recommend that someone fight the fire in their house because of the inherent risk involved, the simple fact is that people do attempt to fight fires and are often successful as shown by the relatively low percentage of fires, $3.4 \%$, are attended by fire departments [62].

Truly understanding the impact that a new technology will have requires considering all the actual outcomes. For example, suppose a new technology significantly increases people's chances of getting out of the structure but at the same time greatly increases the risks to anyone that attempts to fight the fire. This technology could actually increase injuries and death if implemented, and the dangers would not be realized unless all relevant residential behaviors were analyzed.

To better understand the impact of alarm technology on occupant safety, we will examine the impact of an occupant finding a fire while it is still relatively likely that the occupant can successfully fight the fire. After that, we will apply the same analysis to the possibility of occupant evacuation from the fire.

Since this analysis is focused on the impact of the new alarm technology, we will include a fourth alarm. The alarm will simply be a combination of best response of the ionization and photoelectric alarms. In a sense, this alarm will be the "ideal" combination of an ionization and a photoelectric alarm that does not have problems with false alarms. In this report, the alarm will be referred to as the ideal alarm.

\subsubsection{Investigating the Fire}

The only identified source of information about occupants fighting a fire comes from Greene and Andres [40]. While an important resource, it left too many questions unanswered to allow development of a model of occupants finding and fighting the fire. For that reason, any attempt to model the behavior will be kept limited. As discussed in section 3.5, one way to model the behavior is to estimate if the occupants can find the fire early enough to have a high probability of putting the fire out. Since all the fires studied, both flaming and smoldering, have an incipient linear growth period prior to vigorous burning, it is reasonable to assume that when the HRR growth is still linear, the occupants have a relatively high 
probability of successfully fighting the fire, and when vigorous burning starts, occupants have a lower probability of successfully fighting the fire.

As it is not known how long it might take for people to find the fire and because there is a large and likely widely varying population to consider, we will assume a range of estimates for the time it takes to find the fire. The values used will be $(0,30,60,120,180) \mathrm{s}$. Zero seconds will be used to give an upper bound on the percentage of cases with a high probability of successfully firefighting due to the alarm sounding. None of this analysis accounts for people that smell something or see something to indicate the fire before the alarm sounds.

The analysis starts considering each fire type individually to see the impact of individual fire characteristics on each alarm type. The following four tables summarize the percentage of the cases where the alarm sounded with sufficient time before the fires started vigorous burning for each alarm type.

Table 8. Percentage of flaming chair cases where an alarm activation allowed the given time to find the fire

\begin{tabular}{|c|c|c|c|c|}
\hline \multicolumn{5}{|c|}{ Flaming Chair } \\
\hline $\begin{array}{c}\text { Time to Find } \\
\text { Fire }\end{array}$ & Ionization Alarm & $\begin{array}{c}\text { Photoelectric } \\
\text { Alarm }\end{array}$ & New Alarm & Ideal Alarm \\
\hline 0 & $85 \%$ & $52 \%$ & $79 \%$ & $86 \%$ \\
\hline 30 & $82 \%$ & $48 \%$ & $74 \%$ & $82 \%$ \\
\hline 60 & $78 \%$ & $45 \%$ & $70 \%$ & $78 \%$ \\
\hline 120 & $69 \%$ & $38 \%$ & $61 \%$ & $69 \%$ \\
\hline 180 & $60 \%$ & $31 \%$ & $52 \%$ & $60 \%$ \\
\hline
\end{tabular}

Table 9. Percentage of smoldering chair cases where an alarm activation allowed the given time to find the fire.

\begin{tabular}{|c|c|c|c|c|}
\hline \multicolumn{5}{|c|}{ Smoldering Chair } \\
\hline $\begin{array}{c}\text { Time to Find } \\
\text { Fire }\end{array}$ & Ionization Alarm & $\begin{array}{c}\text { Photoelectric } \\
\text { Alarm }\end{array}$ & New Alarm & Ideal Alarm \\
\hline 0 & $95 \%$ & $99 \%$ & $99 \%$ & $\approx 100 \%$ \\
\hline 30 & $94 \%$ & $99 \%$ & $99 \%$ & $\approx 100 \%$ \\
\hline 60 & $94 \%$ & $99 \%$ & $99 \%$ & $99 \%$ \\
\hline 120 & $94 \%$ & $99 \%$ & $99 \%$ & $99 \%$ \\
\hline 180 & $94 \%$ & $99 \%$ & $98 \%$ & $99 \%$ \\
\hline
\end{tabular}


Table 10. Percentage of old mattress cases where an alarm activation allowed the given time to find the fire.

\begin{tabular}{|c|c|c|c|c|}
\hline \multicolumn{5}{|c|}{ Old Mattress (flaming) } \\
\hline $\begin{array}{c}\text { Time to Find } \\
\text { Fire }\end{array}$ & Ionization Alarm & $\begin{array}{c}\text { Photoelectric } \\
\text { Alarm }\end{array}$ & New Alarm & Ideal Alarm \\
\hline 0 & $86 \%$ & $53 \%$ & $79 \%$ & $86 \%$ \\
\hline 30 & $82 \%$ & $49 \%$ & $75 \%$ & $82 \%$ \\
\hline 60 & $78 \%$ & $45 \%$ & $70 \%$ & $78 \%$ \\
\hline 120 & $69 \%$ & $38 \%$ & $61 \%$ & $68 \%$ \\
\hline 180 & $60 \%$ & $32 \%$ & $54 \%$ & $60 \%$ \\
\hline
\end{tabular}

Table 11. Percentage of new mattress cases where an alarm activation allowed the given time to find the fire.

\begin{tabular}{|c|c|c|c|c|}
\hline \multicolumn{5}{|c|}{ New Mattress (flaming) } \\
\hline $\begin{array}{c}\text { Time to Find } \\
\text { Fire }\end{array}$ & Ionization Alarm & $\begin{array}{c}\text { Photoelectric } \\
\text { Alarm }\end{array}$ & New Alarm & Ideal Alarm \\
\hline 0 & $86 \%$ & $53 \%$ & $79 \%$ & $86 \%$ \\
\hline 30 & $83 \%$ & $49 \%$ & $75 \%$ & $83 \%$ \\
\hline 60 & $79 \%$ & $45 \%$ & $70 \%$ & $78 \%$ \\
\hline 120 & $69 \%$ & $39 \%$ & $62 \%$ & $69 \%$ \\
\hline 180 & $60 \%$ & $32 \%$ & $54 \%$ & $60 \%$ \\
\hline
\end{tabular}

The first question that might come to mind is if the ideal alarm is a combination of the photoelectric alarm and the ionization alarm, to what extent does it outperform the ionization alarm for the flaming chair fire and outperform the photoelectric alarm for the smoldering chair? The answer that the ideal alarm can outperform both the ionization and photoelectric alarms. Remember that in an individual CFAST run the performance of the ionization and photoelectric alarms are drawn randomly from distributions. Take for example the flaming chair fire. While the distribution of smoke optical densities for the ionization alarm is generally lower than the photoelectric alarm the two distributions overlap meaning that on occasion the photoelectric alarm can be more sensitive to flaming smoke than the ionization alarm and so the ideal alarm will be more sensitive to flaming smoke than the ionization alarm in that case.

There are several observations from the tables. First is that the flaming chair, old mattress, and new mattress results are very similar. Noting that all three of these fires used the same definition of the linear ramp, it is not surprising that they are similar.

The second observation, which may not obvious in the tables, is that the drop in performance as a function of the time to find the fire is linear. It is easy to see in Figure 29. 


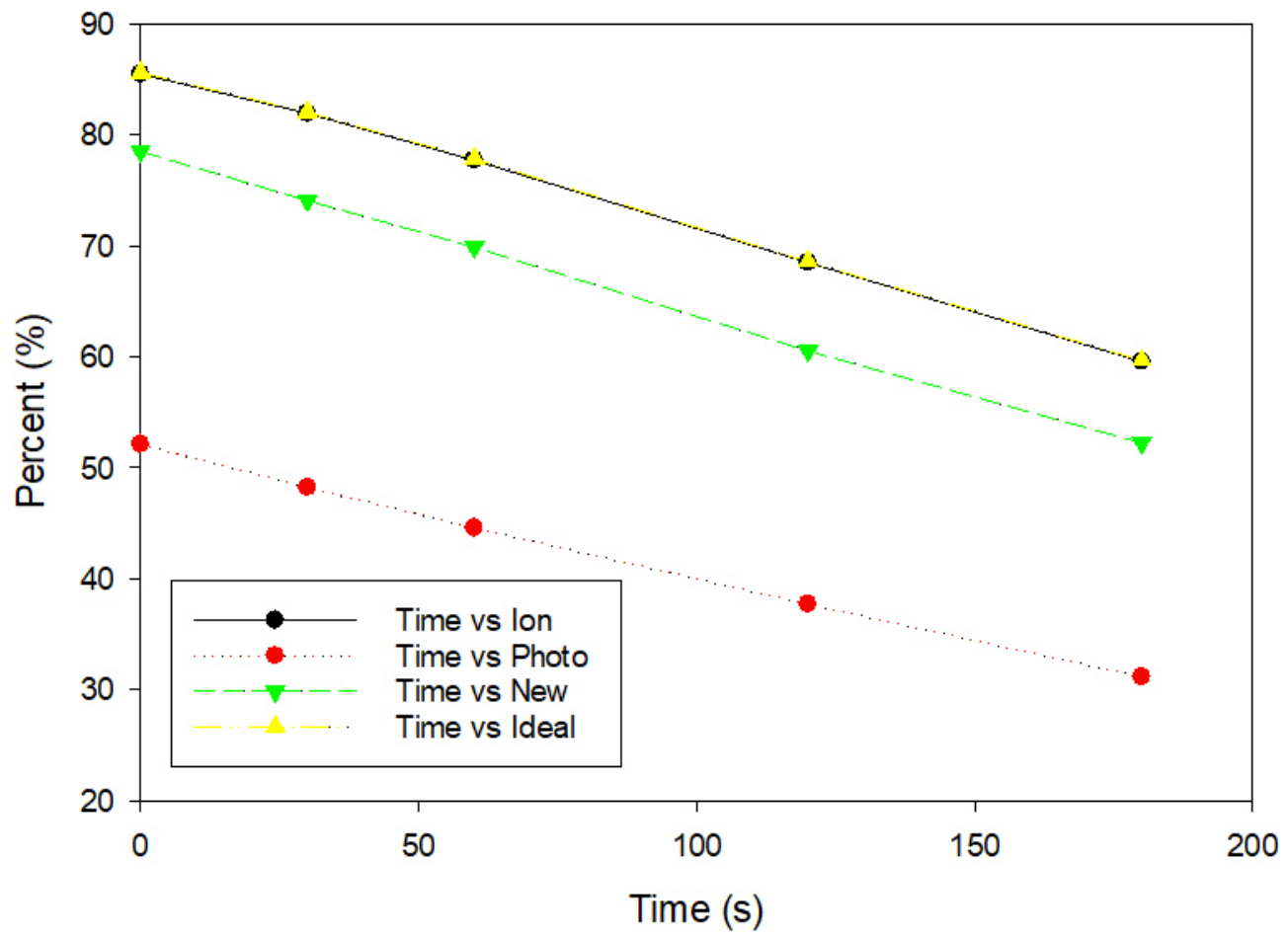

Figure 29 Linear response of the 4 alarms to time required to investigate a fire upon detection.

The third observation is that for each fire type, there is a strong rank order in performance. For the three flaming fire types, the ionization alarm and the ideal alarm give the most opportunity to successfully fight the fire, followed by the new alarm, and with the photoelectric alarms providing the least opportunity. For the smoldering chair, the order is reversed with the photoelectric tied with the ideal for preforming the best and the ionization performing the worst. However, while the photoelectric alarm preforms best for the smoldering chair, the advantage it provides over the ionization alarm is much less than the ionization provides over the photoelectric alarm for the other three fire types.

While the data is informative, there is always a desire for a single number that can be used to evaluate the different alarm types. The first step toward a single value is to combine the impact of the different types of fires. In terms of finding the fire in time to successfully fight it, three of the fire types are nearly identical. Therefore, let us consider them all a single flaming fire type. To generate the table for flaming fire performance, we assume that each fire type is equally likely and average the values. The results can be seen in Table 12 . 
Table 12. Percentage of "flaming fire" cases where an alarm allowed the given time to find the fire.

\begin{tabular}{|c|c|c|c|c|}
\hline $\begin{array}{c}\text { Time to Find } \\
\text { Fire }\end{array}$ & Ion Alarm & Photo Alarm & New Alarm & Ideal Alarm \\
\hline 0 & $86 \%$ & $53 \%$ & $79 \%$ & $86 \%$ \\
\hline 30 & $82 \%$ & $49 \%$ & $75 \%$ & $82 \%$ \\
\hline 60 & $78 \%$ & $45 \%$ & $70 \%$ & $78 \%$ \\
\hline 120 & $69 \%$ & $38 \%$ & $61 \%$ & $69 \%$ \\
\hline 180 & $60 \%$ & $32 \%$ & $53 \%$ & $60 \%$ \\
\hline
\end{tabular}

There are essentially two types of fires, smoldering and flaming. The question then becomes what fraction of the fires are of each type. While it may be possible to infer some data on the fraction of fires that start off flaming and smoldering from the NFIRS database, let us consider all possible combinations to understand the impact of different possibilities.

To examine all possible combinations, we will start by creating 11 data points that are the various weighted averages of the flaming fires times in Table 12 with the smoldering chair fire times from Table 9 for each of the chosen investigation time parameters. The results for the ionization alarms can be seen in Figure 30. The "Time vs Flaming 0\%" curve is the ionization alarms' response to just smoldering fires while the "Time vs Flaming 100\%" is the ionization alarms response to only flaming fires. The curves in between are the ionization alarms response to a scenario where $x \%$ of the fires are flaming fires and $(1-x) \%$ of the fires are smoldering fires.

This still does not provide a single number that is indicative of the alarm's performance for the particular distribution of smoldering and flaming fires since there is still an impact of the time taken to investigate the fire. One way of coming up with a single value is to pick a representative value for the investigation time. For example, a value of $60 \mathrm{~s}$ to investigate and find the fire could be chosen for a particular distribution of flaming versus smoldering fires. One might suggest averaging the scenarios as we averaged the fires but that would simply be the $50 \%$ flaming fires and $50 \%$ smoldering fires specified in the fire scenario definitions. 


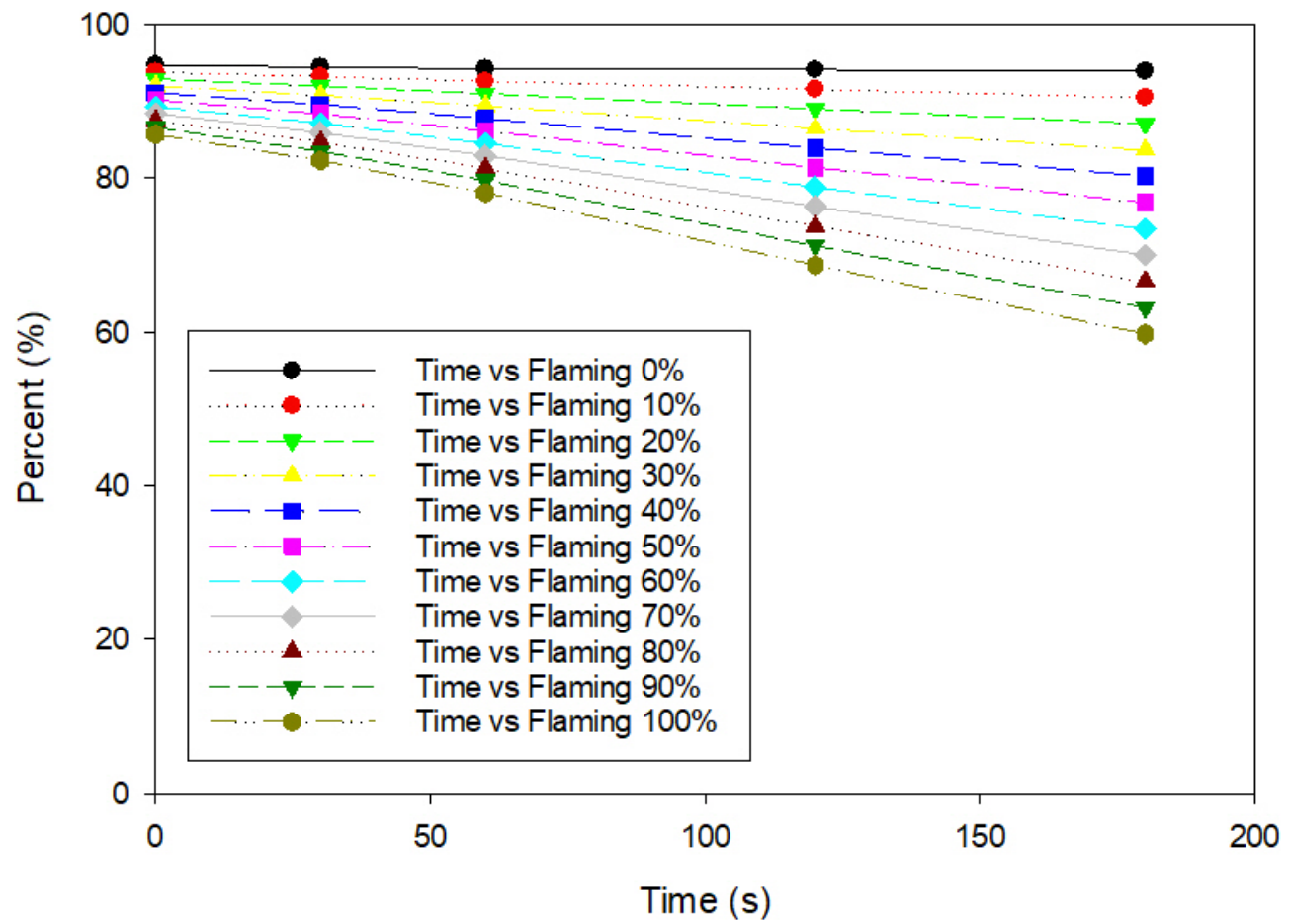

Figure 30 Percent of ionization alarm activation for different times to find fire and 11 combinations of smoldering and flaming fires

Suppose that we had some information about how fast people find the fire or at least were willing to make assumptions about the speed people find fires. With that information, it would be possible to combine the data in any one of the three tables of scenarios to create a single performance number estimating the probability that occupants that go looking for the fire will find it and still have a high probability of successfully fighting the fire. This still would not be an estimation of how often occupants put out the fire. That would require estimates from data or assumptions about

1. the probability of people looking for the fire,

2. the probabilities of when they find the fire in either stage, they attempt to fight it, and finally

3. the probabilities of successfully fighting the fire for either stage of the fire.

Also note that this assumes that all probabilities discussed here are the same for flaming fires and smoldering fires, which may not be the case.

Without data to estimate these probabilities, we can do the same thing we did with the scenarios and pick a range of possible results. Step one is to consider what the statistics are saying. Consider Table 12 and the 30 s row. It says that for some percentage of the fires, each type of alarm will provide at least $30 \mathrm{~s}$ of time. So, the ionization alarm will provide at least $30 \mathrm{~s}$ of time to find the fire before vigorous burning starts in $82 \%$ of cases. Let us assume 
that the amount of time an occupant needs to find the fire is independent of the type of fire, so $x$ percent of the time occupants find the fire in $30 \mathrm{~s}$. If we take the percentage of people that can find the fire in $30 \mathrm{~s}$ times the percentage of fires that give at least $30 \mathrm{~s}$ of time, we have the percentage of fires where people successfully find the fire in $30 \mathrm{~s}$. Note that the independence of these assumptions is very important. If the time it takes to find the fire is dependent on the type of fire, the calculation is more difficult and requires additional information or assumptions.

Assume three possible distributions of how fast people find the fire as given in Table 13. One distribution is weighted toward people investigating and finding the fire quickly (fast), one is weighted toward people taking a longer amount of time to find the fire (slow), and one is not weighted one way or the other (medium).

Table 13 Three distributions of the faction of people who find the fire in a particular amount of time

\begin{tabular}{|c|c|c|c|c|}
\hline & $\begin{array}{c}\text { Finding Fire in } \\
30 \mathrm{~s}\end{array}$ & $\begin{array}{c}\text { Finding Fire in } \\
60 \mathrm{~s}\end{array}$ & $\begin{array}{c}\text { Finding Fire in } \\
120 \mathrm{~s}\end{array}$ & $\begin{array}{c}\text { Finding Fire in } \\
180 \mathrm{~s}\end{array}$ \\
\hline Fast & 0.4 & 0.3 & 0.2 & 0.1 \\
\hline Medium & 0.25 & 0.25 & 0.25 & 0.25 \\
\hline Slow & 0.1 & 0.2 & 0.3 & 0.4 \\
\hline
\end{tabular}

If we create graphs for the other three alarms like was done for the ionization alarms in Figure 30 and then combine the percent successes for the four times using the values in Table 13 we can generate three graphs as seen in Figure 31, Figure 32, and Figure 33. Note that the three figures look very similar. The fast, medium and slow distributions have little effect on the rankings. That may not always be true but is here. It also means that at least for investigating, a single representative value would perform just as well. Also note how the ionization, new, and ideal alarms are relatively close for all distributions of fires, but the photoelectric alarms perform significantly worse for distributions of about $20 \%$ flaming and higher. 


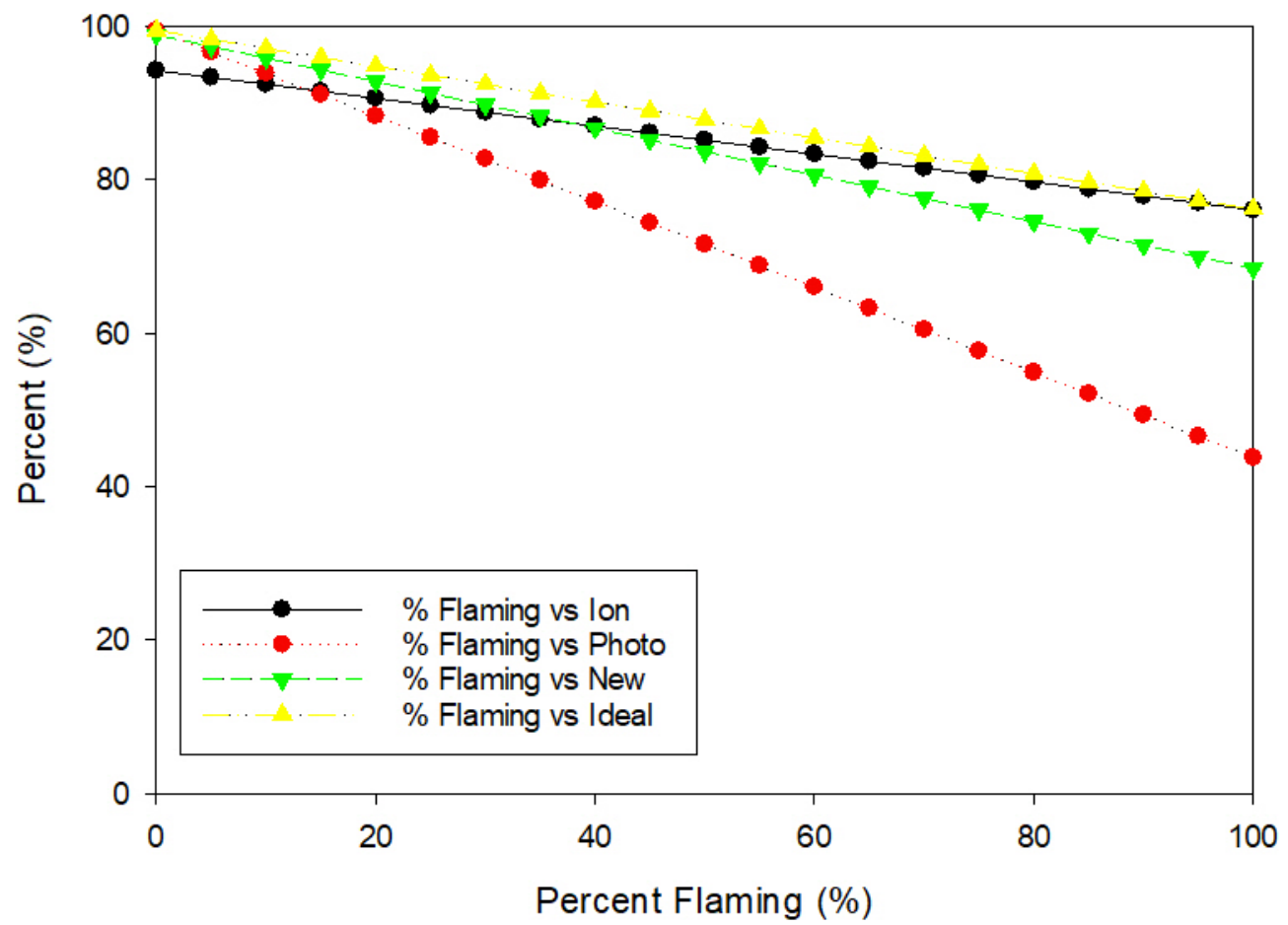

Figure 31 Performance of successfully finding the fire for 4 alarms across all distributions of flaming and smoldering fires for the fast distribution of finding fires 


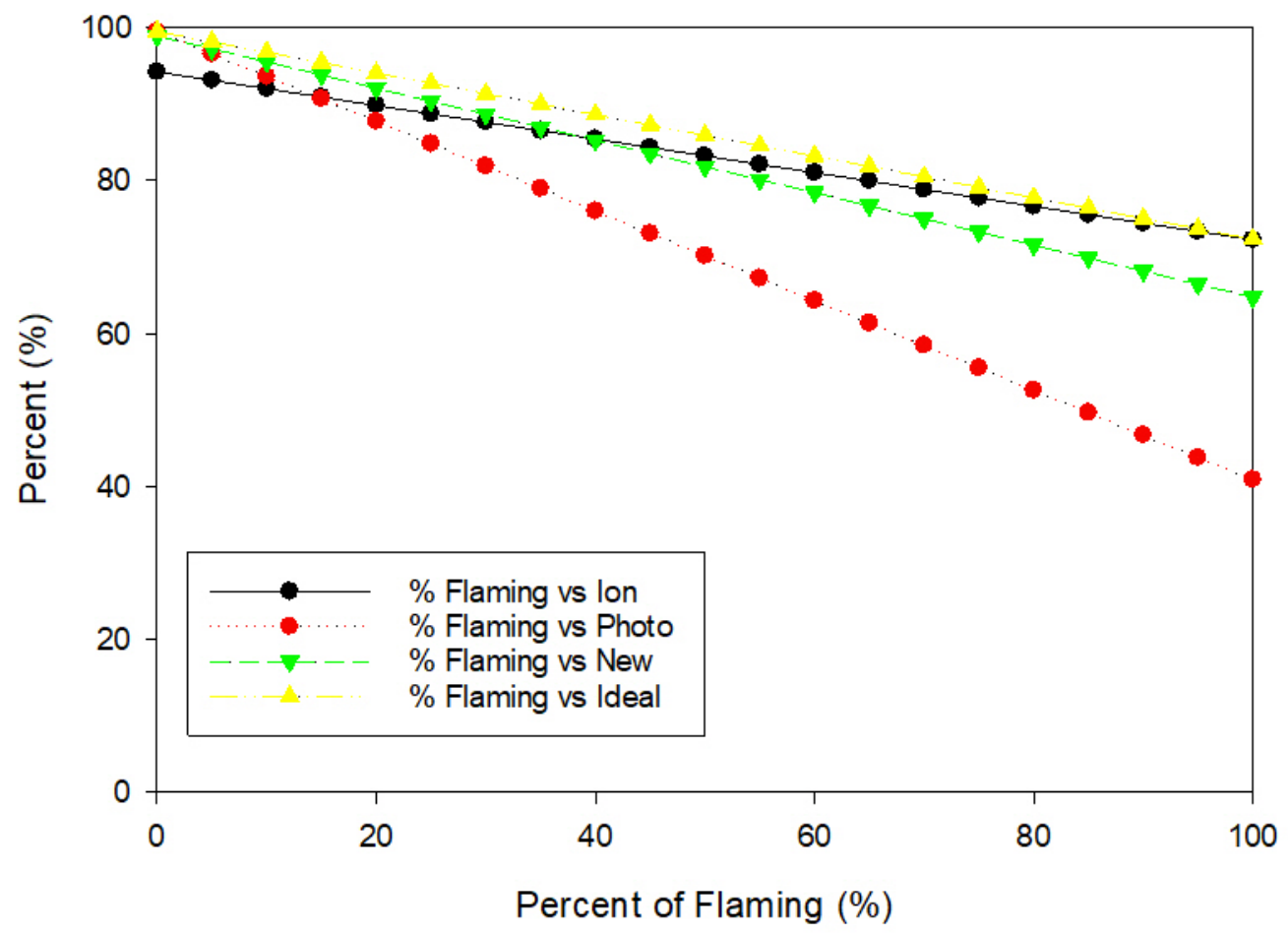

Figure 32 Performance of successfully finding the fire for 4 alarms across all distributions of flaming and smoldering fires for the medium distribution of finding fires. 


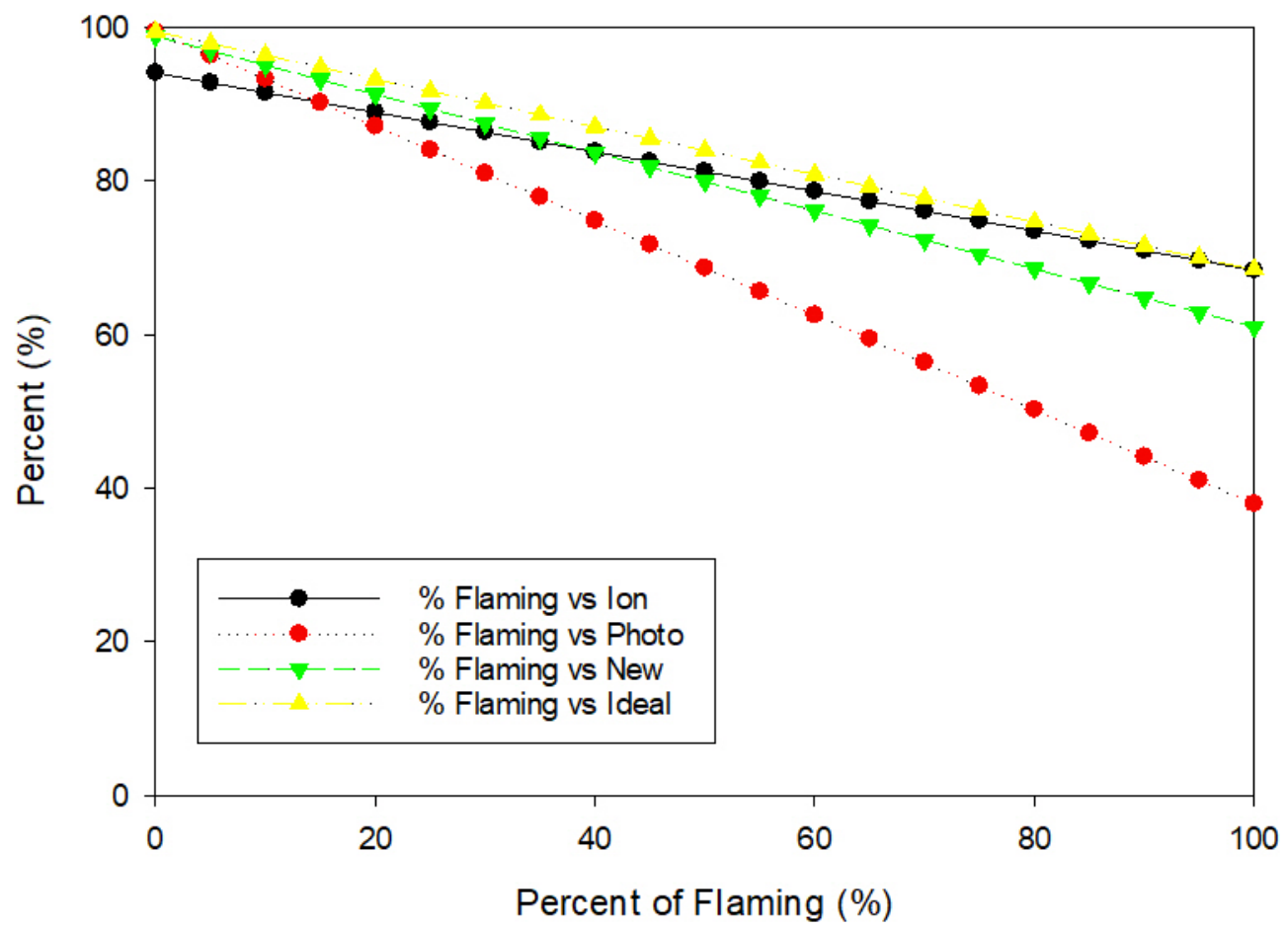

Figure 33 Performance of successfully finding the fire for 4 alarms across all distributions of flaming and smoldering fires for the slow distribution of finding fires.

\subsubsection{Evacuation}

The analysis of the impact of the new alarms on evacuation will follow the same pattern as the previous analysis of the impact of the new alarms on the possibility of successfully fighting the fire. However, because it is following the same pattern and because there is so much more data, only a representative sample of the data will be included in the section.

This analysis is not done with the original 57500 cases because the smoke criteria had not been added to the analysis at the point the cases were run. Because we discarded the output of each case after we collected the identified data, it was not possible to go back and collect the smoke data. This analysis is done with a set of 11500 cases that did include the smoke criteria.

The first step is to define the conditions of safe egress. Without a model of residential egress and using a random set of floor plans, there is no realistic way to track occupants through a residence to see if they can safely egress. Rather, a set of indicators of the overall safety of the structure (with respect to occupant safety during egress) is used. At all times, both the thermal Fractional Effective Dose (FED) and toxic gas FED is calculated at a set location in each room (not including the fire room). The time is recorded when the first compartment other than the fire compartment reaches a trigger value (indicative of untenable conditions within the compartment) for either the thermal or toxicity FED. The time the first compartment (other than the fire room) reaches an optical density of $0.25 \mathrm{~m}^{-1}$ or greater is also recorded. Then it is simply a process of determining if the difference between the alarm 
activation time and the time the target FED is reached is greater than the particular egress time that is assumed. The FED values we will examine are 0.3, 0.5, 1.0. Bukowski et. al. [60] found that for a young family, an egress time of $90 \mathrm{~s}$ was a reasonable time. As with the analysis of time investigation, we will use several multiples of $90 \mathrm{~s}$ to understand the range of possible impacts. Table 14 shows the probability of egressing from a flaming chair fire for a range of tenability limits, not accounting for smoke obscuration.

Table 14 Cases with Successful Egress for the flaming chair fire with a target FED $=0.3,0.5$, and 1.0 and not accounting for smoke

\begin{tabular}{|l|l|l|l|l|}
\hline Time & Ion Alarm & Photo Alarm & New Alarm & Ideal Alarm \\
\hline
\end{tabular}

$\mathrm{FED}=0.3$

\begin{tabular}{|c|c|c|c|c|}
\hline 0 & $97 \%$ & $67 \%$ & $95 \%$ & $97 \%$ \\
\hline 45 & $92 \%$ & $47 \%$ & $88 \%$ & $93 \%$ \\
\hline 90 & $87 \%$ & $28 \%$ & $78 \%$ & $87 \%$ \\
\hline 180 & $69 \%$ & $6 \%$ & $53 \%$ & $69 \%$ \\
\hline 270 & $38 \%$ & $\approx 0 \%$ & $22 \%$ & $38 \%$ \\
\hline
\end{tabular}

$\mathrm{FED}=0.5$

\begin{tabular}{|c|c|c|c|c|}
\hline 0 & $98 \%$ & $79 \%$ & $97 \%$ & $98 \%$ \\
\hline 45 & $95 \%$ & $61 \%$ & $93 \%$ & $95 \%$ \\
\hline 90 & $90 \%$ & $40 \%$ & $84 \%$ & $90 \%$ \\
\hline 180 & $76 \%$ & $16 \%$ & $63 \%$ & $76 \%$ \\
\hline 270 & $50 \%$ & $3 \%$ & $36 \%$ & $50 \%$ \\
\hline
\end{tabular}

$\mathrm{FED}=1.0$

\begin{tabular}{|c|c|c|c|c|}
\hline 0 & $99 \%$ & $90 \%$ & $99 \%$ & $99 \%$ \\
\hline 45 & $97 \%$ & $76 \%$ & $96 \%$ & $97 \%$ \\
\hline 90 & $93 \%$ & $57 \%$ & $89 \%$ & $94 \%$ \\
\hline 180 & $83 \%$ & $31 \%$ & $72 \%$ & $83 \%$ \\
\hline 270 & $64 \%$ & $12 \%$ & $52 \%$ & $64 \%$ \\
\hline
\end{tabular}

Figure 34 shows that unlike investigation, the relationship between the time it takes to escape and the percentage of cases each alarm gives at least that amount of time is not linear. The rank order is what would be expected. Notice that as the time required to evacuate grows, the difference in the percentage of alarms activating for the ionization and new alarms grows. The other flaming fires have similar results. It is also interesting that the performance for the ionization, new and of course ideal alarms does not degrade as quickly as the time to escape grows. However, the photoelectric alarm's performance degrades more quickly in comparison. 


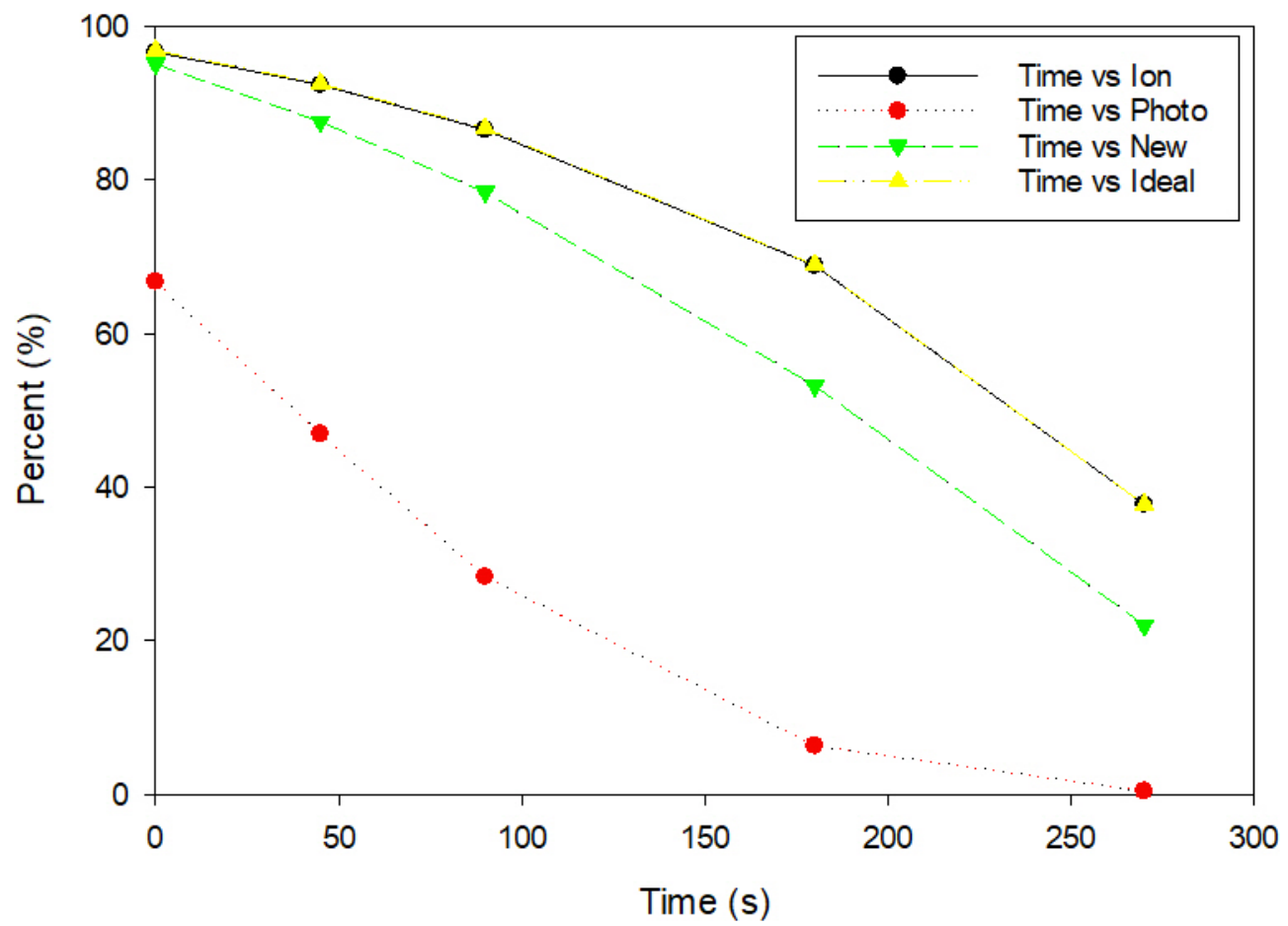

Figure 34 For flaming chair fire, percentage of cases with successful egress for FED $=0.3$ and not accounting for smoke.

The smoldering chair fire is significantly different from the flaming fires. As an example, Table 15 shows the performance of the three alarms for a target FED $=0.3$. The photoelectric, new, and ideal alarms perform almost the same, but the ionization alarm is significantly worse. Also, the ionization alarm's performance decreases as the egress time goes up where as the photoelectric and new alarms stay nearly unchanged.

Table 15 Cases with Successful Egress for the smoldering chair fire with a target FED $=0.3$ and not accounting for smoke.

\begin{tabular}{|c|c|c|c|c|}
\hline Time & $\begin{array}{c}\text { Ion Alarm } \\
\text { at FED }=0.3\end{array}$ & $\begin{array}{c}\text { Photo Alarm at } \\
\text { FED }=0.3\end{array}$ & $\begin{array}{c}\text { New Alarm } \\
\text { at FED }=0.3\end{array}$ & $\begin{array}{c}\text { Ideal Alarm at } \\
\text { FED =0.3 }\end{array}$ \\
\hline 0 & $79 \%$ & $99 \%$ & $98 \%$ & $99 \%$ \\
\hline 45 & $77 \%$ & $99 \%$ & $98 \%$ & $99 \%$ \\
\hline 90 & $74 \%$ & $99 \%$ & $97 \%$ & $99 \%$ \\
\hline 180 & $70 \%$ & $98 \%$ & $97 \%$ & $99 \%$ \\
\hline 270 & $66 \%$ & $98 \%$ & $97 \%$ & $98 \%$ \\
\hline
\end{tabular}

As can be seen in Figure 35, the relationship is linear, with the drop in performance less pronounced for the smoldering fires. This is because there is so much additional time before untenable conditions are reached, even for the ionization alarms, that the egress time required is not an important factor. 


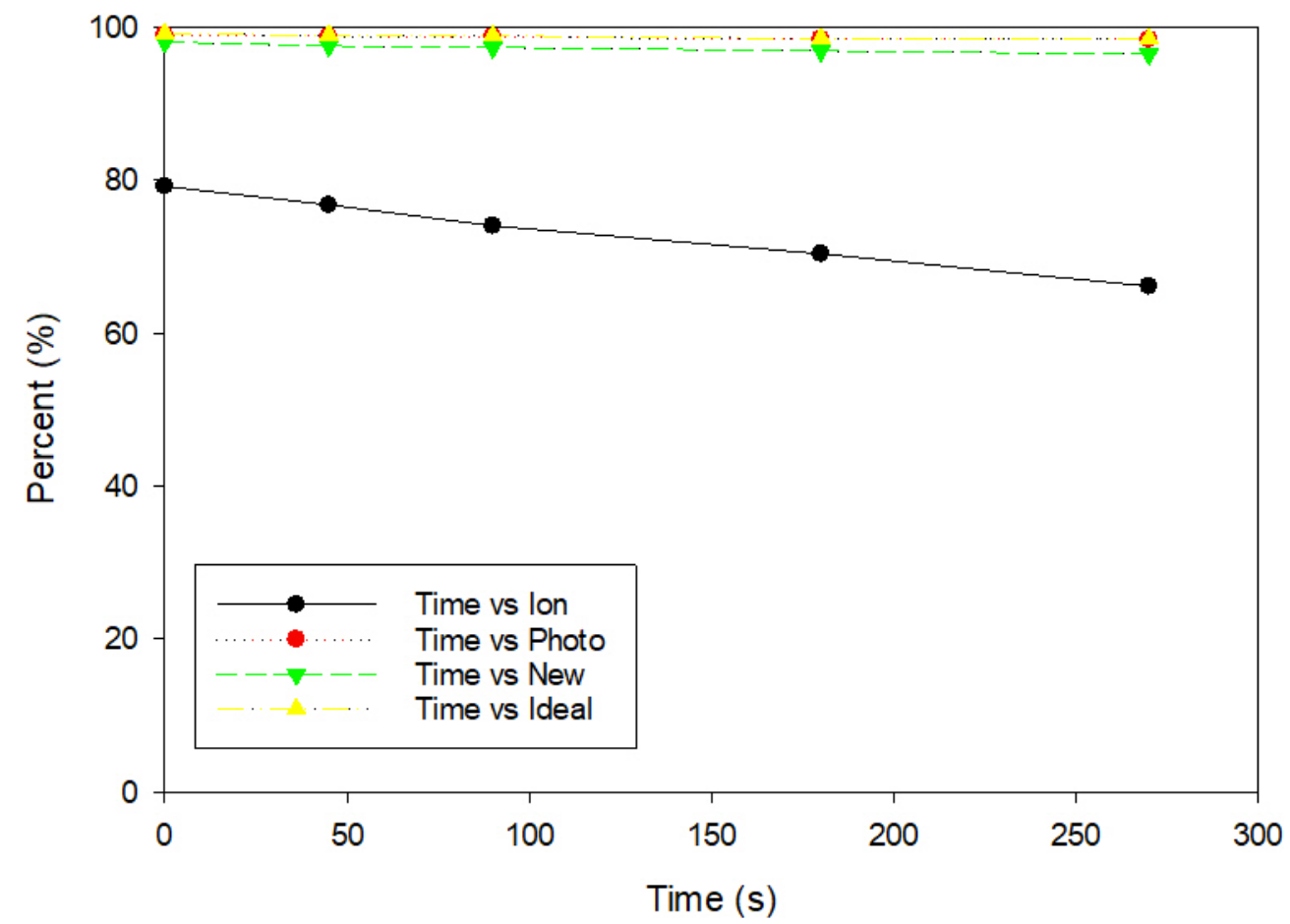

Figure 35 For smoldering chair fire, percentage of cases with successful egress for FED $=0.3$ not including the impact of smoke tenability.

Now that we have looked at the differences between the data for investigation and the data for evacuations, we can follow the steps in section 4.4.1 to generate the graphs that describe the performance of the four alarms across the range of combinations of smoldering and flaming fires. As with investigating the fire, the performance does not vary significantly based on the distribution of times we use so we will just consider the medium distribution of times.

Figure 36 shows the overall performance of the four types of alarms to allow enough time to either find and fight the fire or to allow enough time to evacuate. Figure 36 shows that the ionization alarm performs about the same regardless of the fire type. This is driven by the fact that there is little drop off in performance for smoldering fires at the longer egress times while for the flaming fires the performance does drop, especially by $270 \mathrm{~s}$, Figure 34 . The photoelectric alarm's poor response for flaming fires, especially at longer egress times, means that the new alarm is the best performer for a majority of the scenarios, and it is not all that bad for even the $100 \%$ flaming fires. Also, notice how close the new alarm is to the ideal alarm's performance. Unless it is known that the environment will have overwhelmingly more flaming ignition fires to smoldering fires, the new alarm is the best performing alarm. 


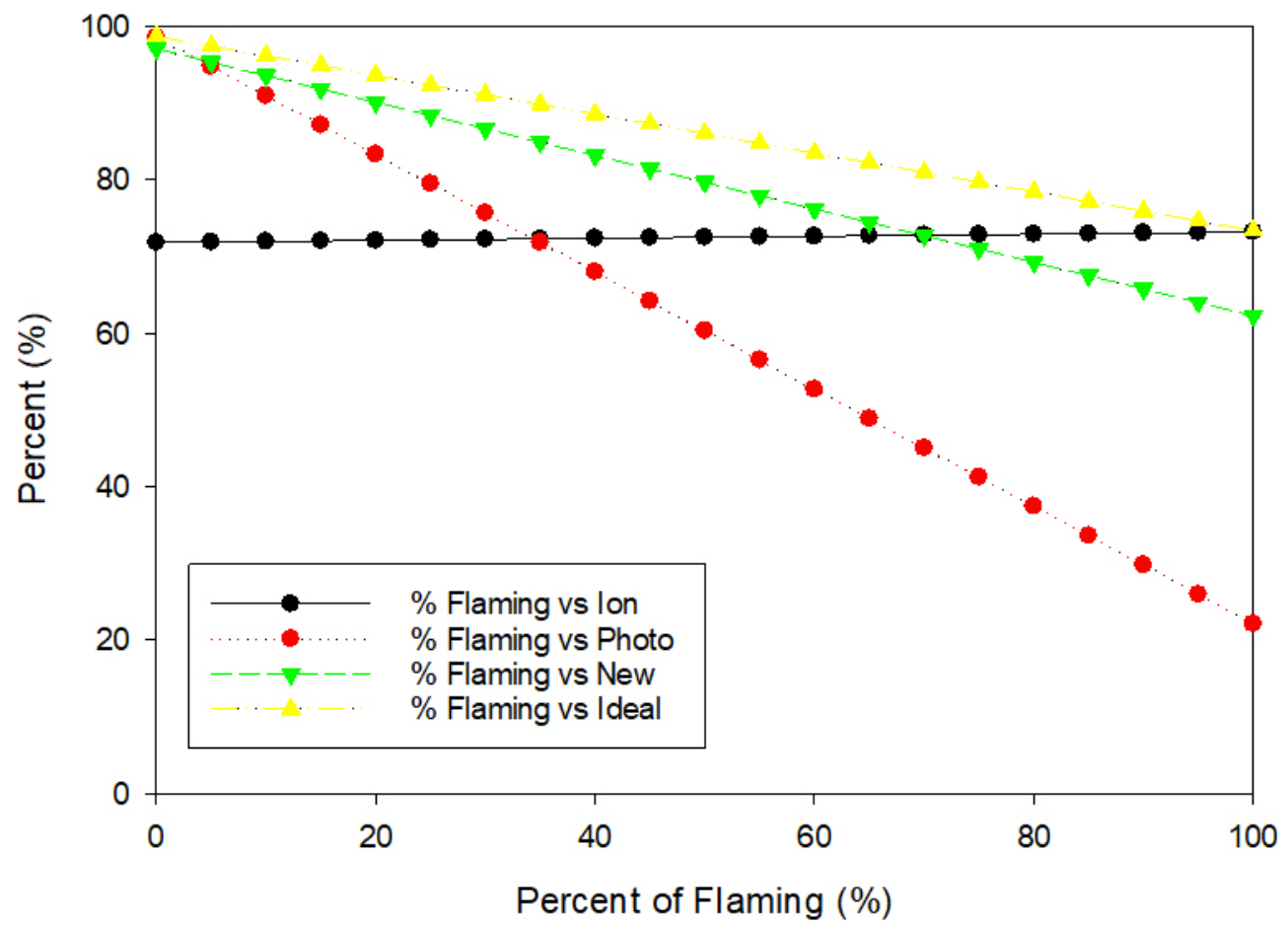

Figure 36 Overall performance of 4 alarms over range of smoldering and flaming fires, $\mathrm{FED}=0.3$ and no smoke considered, using the medium speed distribution.

Now we consider the impact that the strict limit on smoke obscuration has on egress. Figure 37 shows the impact of considering smoke. on the percentage of cases with successful egress for the flaming chair Notice how much the performance of every alarm at every time is reduced compared to Figure 34. Basically, smoke is the first criteria reached in virtually every case. For that reason, there is little point to considering the FED $=0.5$ and FED $=1.0$ cases. inI fact they are essentially the same as the FED $=0.3$ case. 


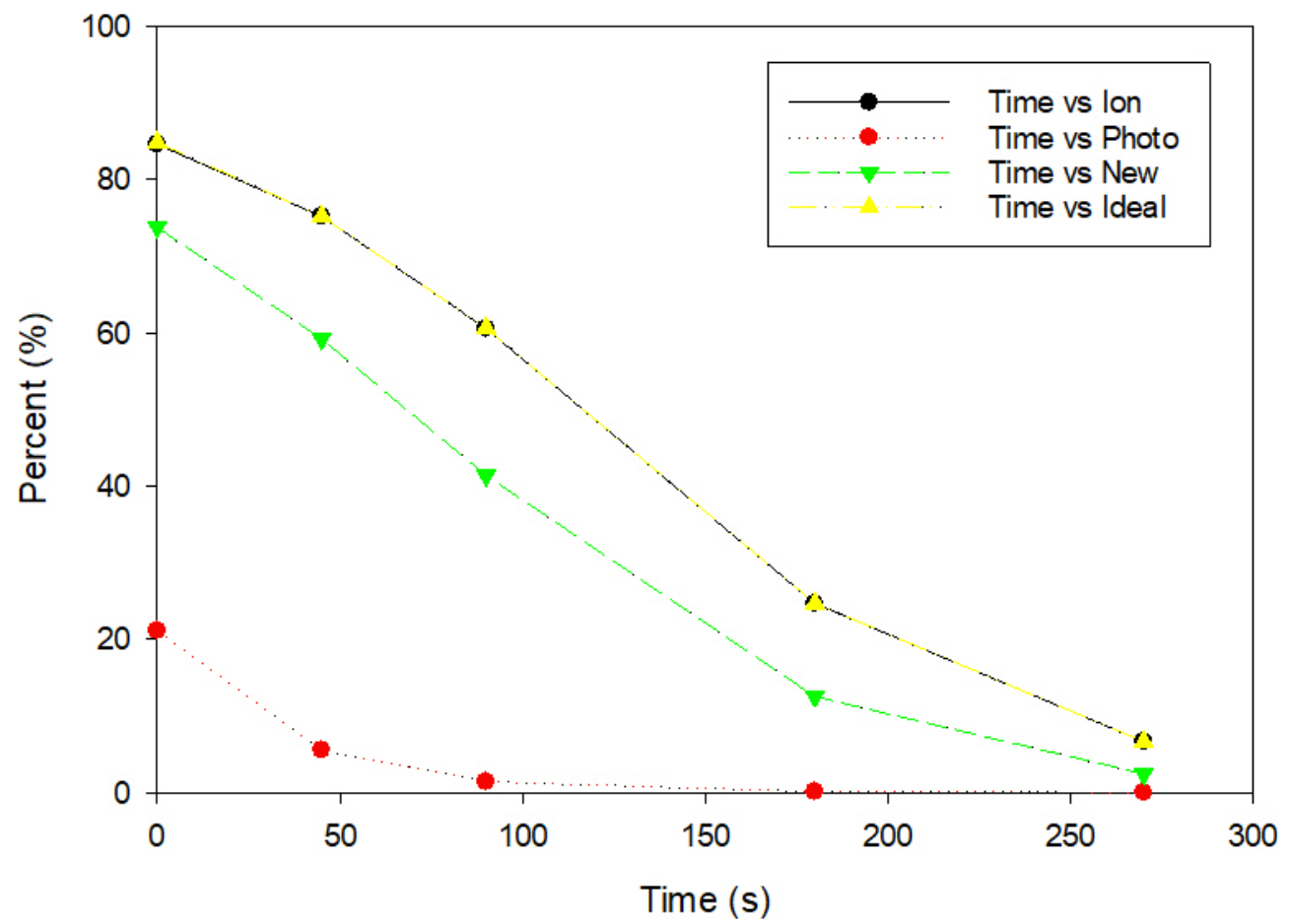

Figure 37 For flaming chair fire, percentage of cases with successful egress for FED $=0.3$ and smoke.

The same impact is seen for the smoldering chair. Without smoke in Figure 35, the ionization alarm is not the best performing alarm, but it still provides sufficient time for egress in over $65 \%$ of the cases. Whereas when considering smoke, Figure 38 shows the ionization alarms with a very low percentage of successful evacuations. Without smoke, the new alarm performed almost as well as the photoelectric alarm (for the smoldering chair with an FED of 0.3 ), but the new alarm is significantly worse than the photoelectric alarm when accounting for smoke. Even the photoelectric alarm performance is much reduced compared to when smoke was not considered. 


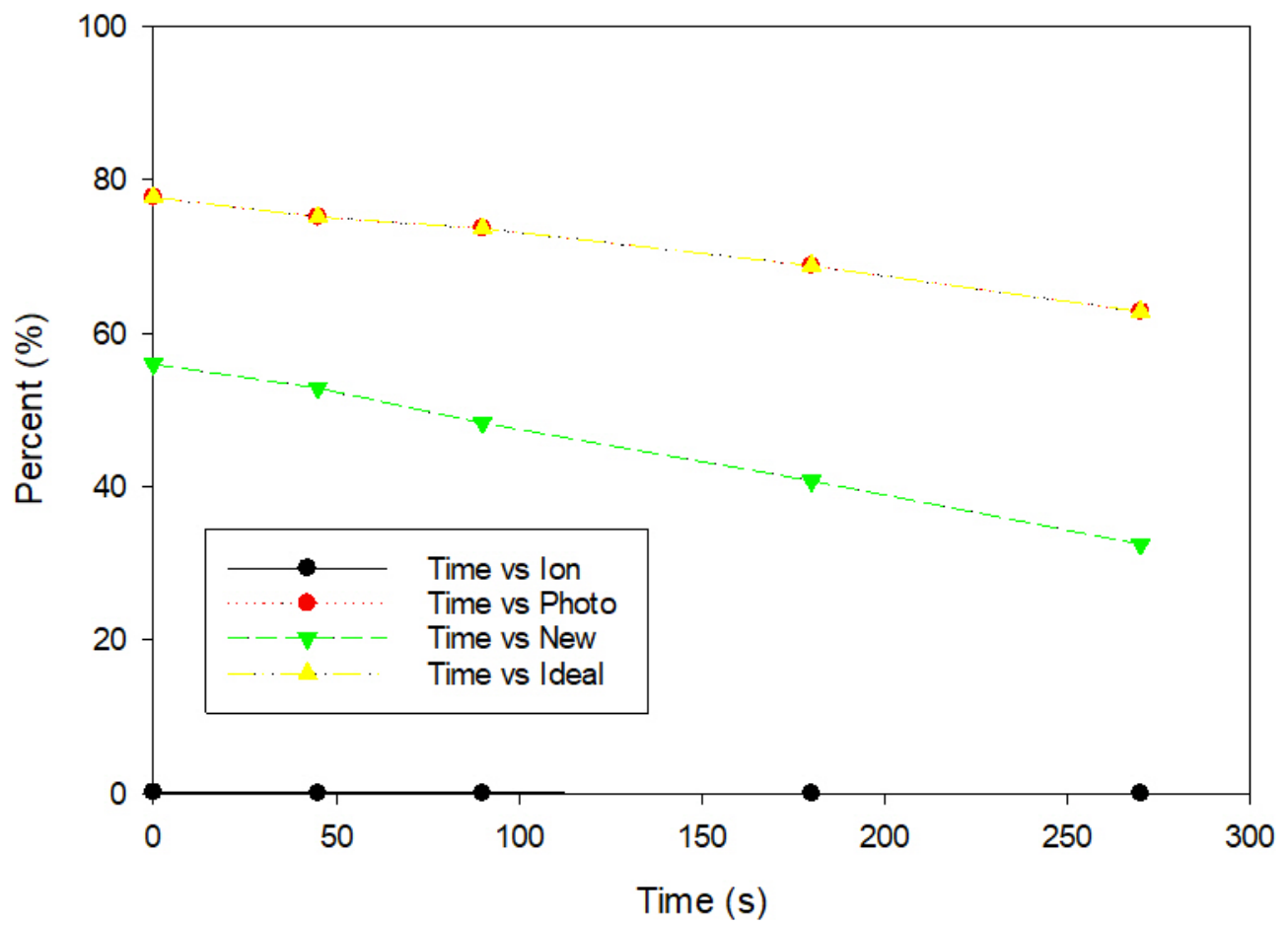

Figure 38 For smoldering chair fire, percentage of case with successful egress for FED $=0.3$ when including the tenability impact of smoke.

When smoke was not considered, there was so much time between the photoelectric alarm's activation for smoldering fire cases (Figure 35) and the time when any room reached FED = 0.3 that evacuation was successful in almost $100 \%$ of the cases.

Figure 39 which shows that the overall performance of the different alarms to smoldering fires is different from the case where smoke was not considered, Figure 35. The delay between when the photoelectric alarms and the new alarms sounded is relatively small compared to the time to FED $=0.3$, so that there is very little difference between the new and photoelectric alarm performance. Even the ionization alarms are successful almost two thirds of the time.

When the smoke criterion is added, performance of all of the alarms is reduced. The performance advantage of the new alarm is smaller than when the smoke criteria is not considered. It is still the case that if there is not a lot of confidence in the composition of the types of fires that will be faced, the new alarm is still the best option. The problem with the individual ionization and photoelectric alarms is that for one type of fire, each performs very poorly. 


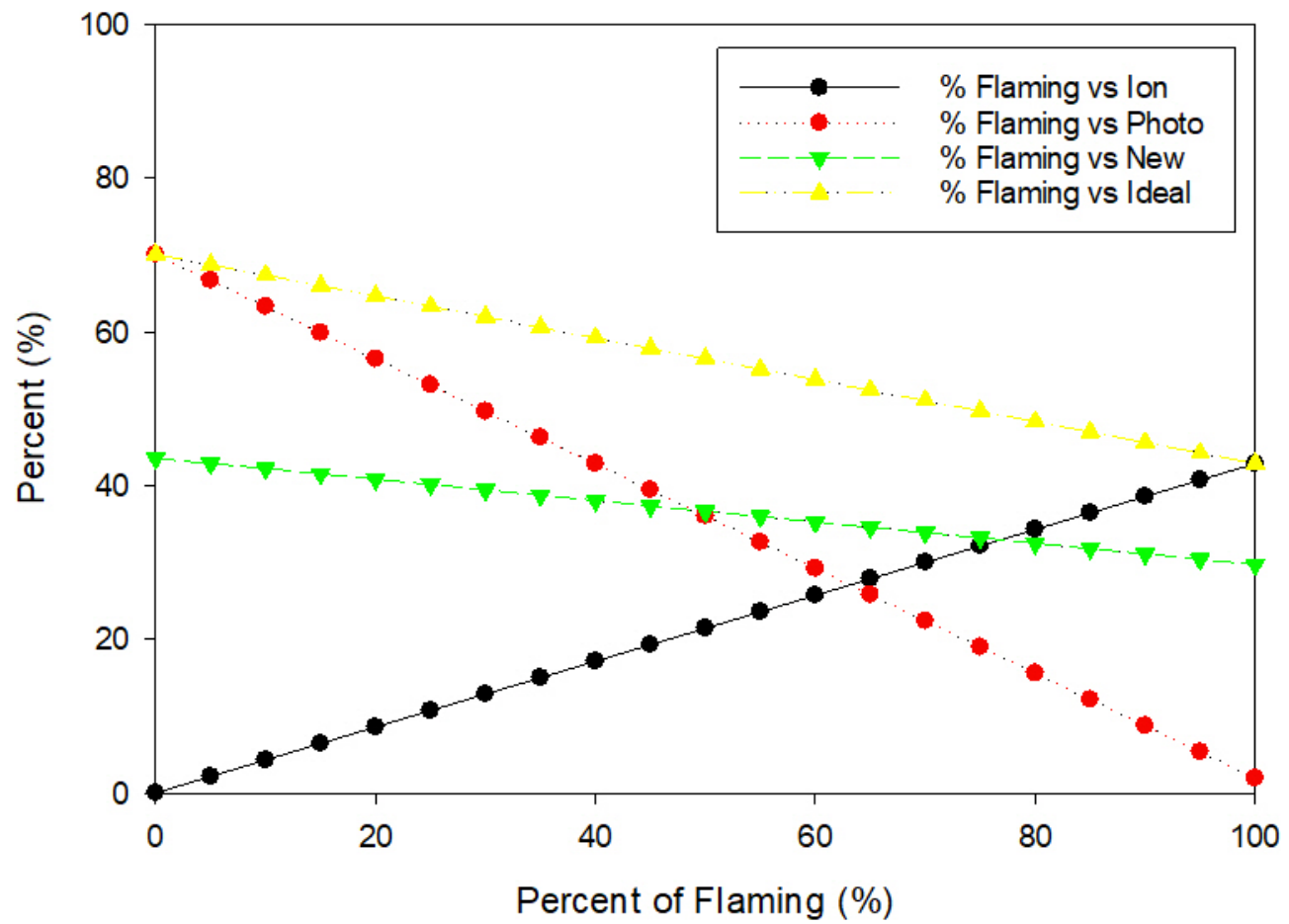

Figure 39 Overall performance of 4 alarms over range of smoldering and flaming fires, FED $=0.3$ and smoke considered, using the medium speed distribution.

\subsubsection{Sensitivity to Estimates of New Alarm Performance}

It is important to remember that the parameters for the new alarm's performance are hypothetical estimates and not based on any actual testing. Given that it is important to understand the impact of those estimates on the results, we need to look at the sensitivity of the results to the new alarm parameters.

Because the greatest difference in performance between the new alarm and the ideal alarm is in the egress analysis when considering smoke, the analysis focuses on that case. The sensitivity was examined by changing the mean values for the distributions used for the flaming and smoldering smoke activation value for the new alarms by $\pm 20 \%$. The ionization and photoelectric alarms values were not changed. To keep the analysis straightforward we either increased or decreased both mean values together. More than 11500 cases were run for both the $20 \%$ increase and $20 \%$ reduction.

Just to be clear, a $20 \%$ reduction in the mean for flaming fires means on average the alarms activate to smoke with an optical density that is $80 \%$ of the base case, which means it activates faster. A $20 \%$ increase in the mean means the alarms activate slower than the base case.

With the $20 \%$ increase in the means, we arrive at Figure 40. The new alarm's performance is significantly reduced compare to what is seen in Figure 39. At no point along the range of the 
distribution of fires is the new alarm performance the best, and for a small range in the middle of the figure it is not even the second best alarm. Even with no information about the distribution of the types of fires, it is not as clear as before that the new alarm is the best option.

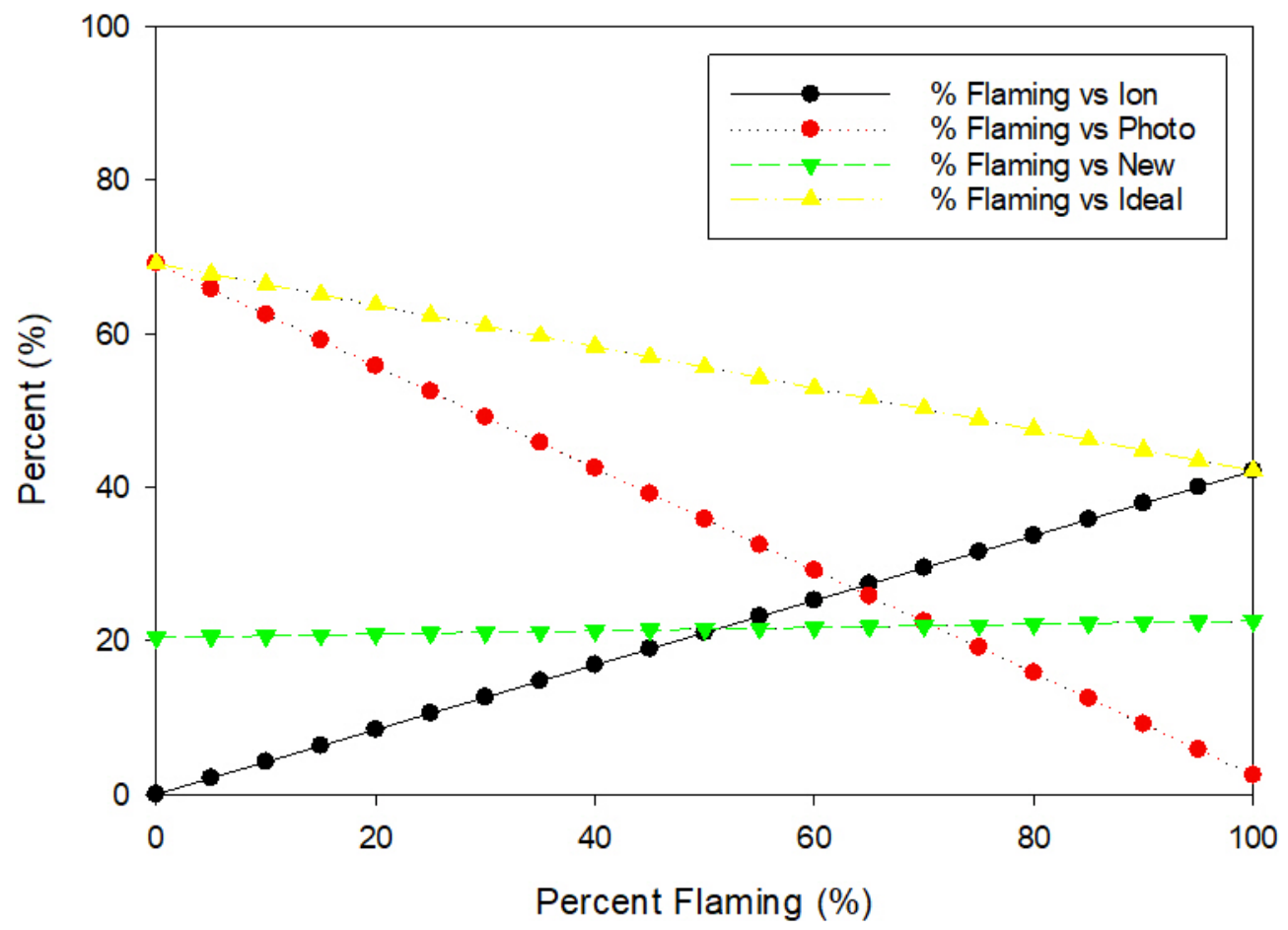

Figure 40 Overall performance of 4 alarms over range of smoldering and flaming fires, $\mathrm{FED}=0.3$ and smoke considered, using the medium speed distribution and increasing the mean smoke activation value for the new alarm by $20 \%$.

Now consider the case where the mean smoke activation value is reduced by $20 \%$, meaning the alarm is $20 \%$ more sensitive. Figure 41 shows that by reducing the mean of the distribution by $20 \%$ the new alarm performance increases significantly, very close to the ideal alarm. In fact, while the performance without smoke is not shown, reducing the mean by $20 \%$, makes the new alarm almost indistinguishable from the ideal alarm's performance for an FED of 0.3 without smoke. 


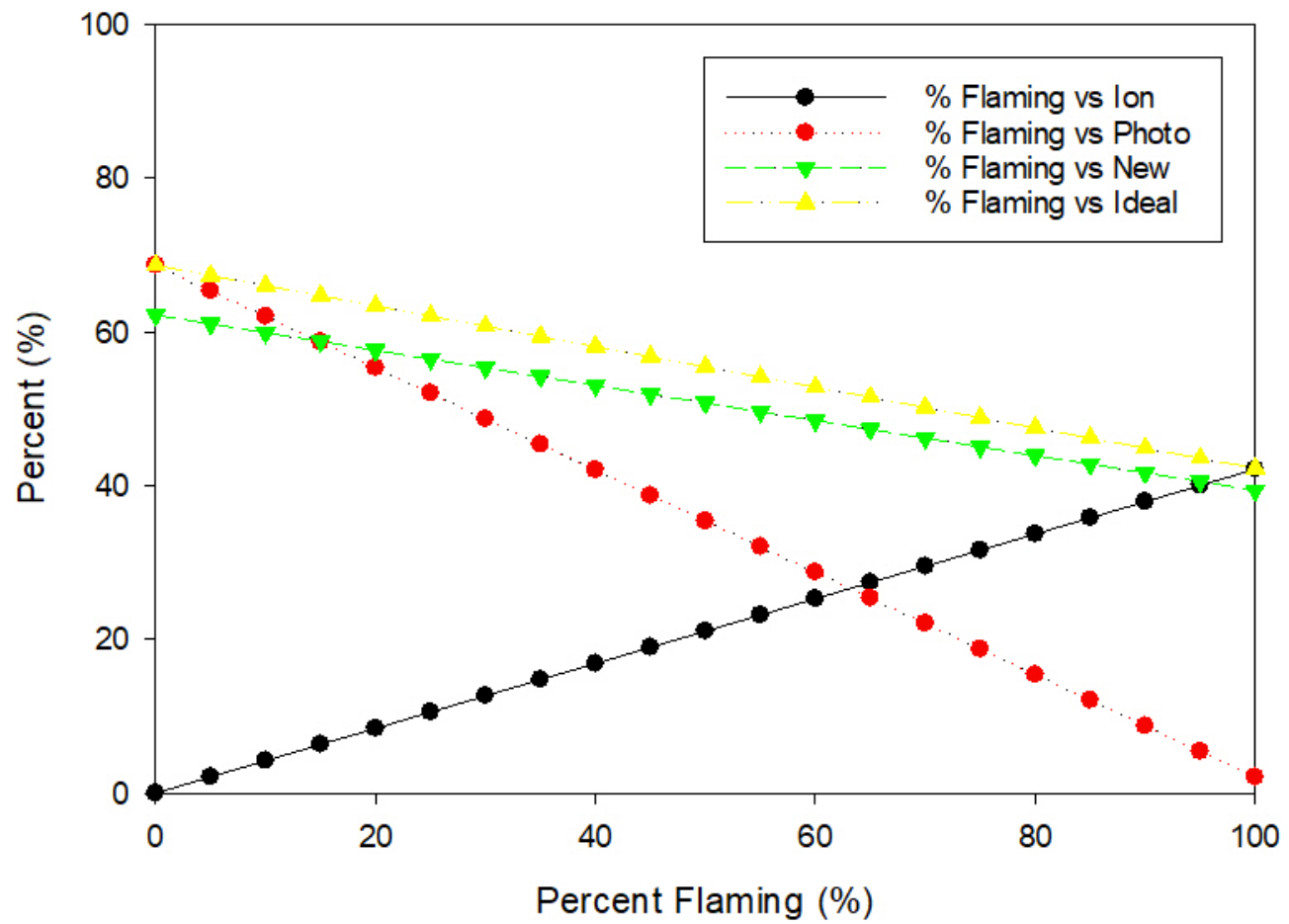

Figure 41 Overall performance of 4 alarms over range of smoldering and flaming fires, FED $=0.3$ and smoke considered, using the medium speed distribution and decreasing the mean new smoke alarm activation value for the new alarm by $20 \%$.

For the fires that quickly reach conditions defined as hazardous to occupants, the performance of alarms, both on an absolute scale and relative scale, is dependent on the alarm sensitivity to the kind of smoke being generated. The question then becomes how quickly fires reach hazardous conditions which would require additional analysis and data on the actual distributions of fires.

\subsection{Highly Toxic Fires}

One of the assumptions that was made was to have all the fires burn with a chemical formula that produced toxic gases that were consistent with the fires measured by Cleary [58]. The question is how much of an impact that assumption had on the results. To look at the impact of very toxic fires, we ran more than 11500 cases that produced 10 times the HCN as the base cases.

Using the high toxicity cases, we generate the same graphs used in proceeding sections for the medium speed distribution, with an FED of 0.3 and accounting for the smoke criteria, as shown in Figure 42. For most of the range of fire distributions, the photoelectric alarm is the best and the new alarm significantly under performs the ideal alarm. This is a bit misleading because the smoldering fires are so much slower than the flaming fires, the photoelectric alarms have a time to respond while not even the ionization alarms have time to respond for the flaming fires. Note that this is a very low FED value that is being used and it is applied to 
all rooms. Thus, the graph does not imply that the vast majority will die in a highly toxic flaming fire. This is a good point to look at the graph for an FED of 1.0, which hasn't been considered before.

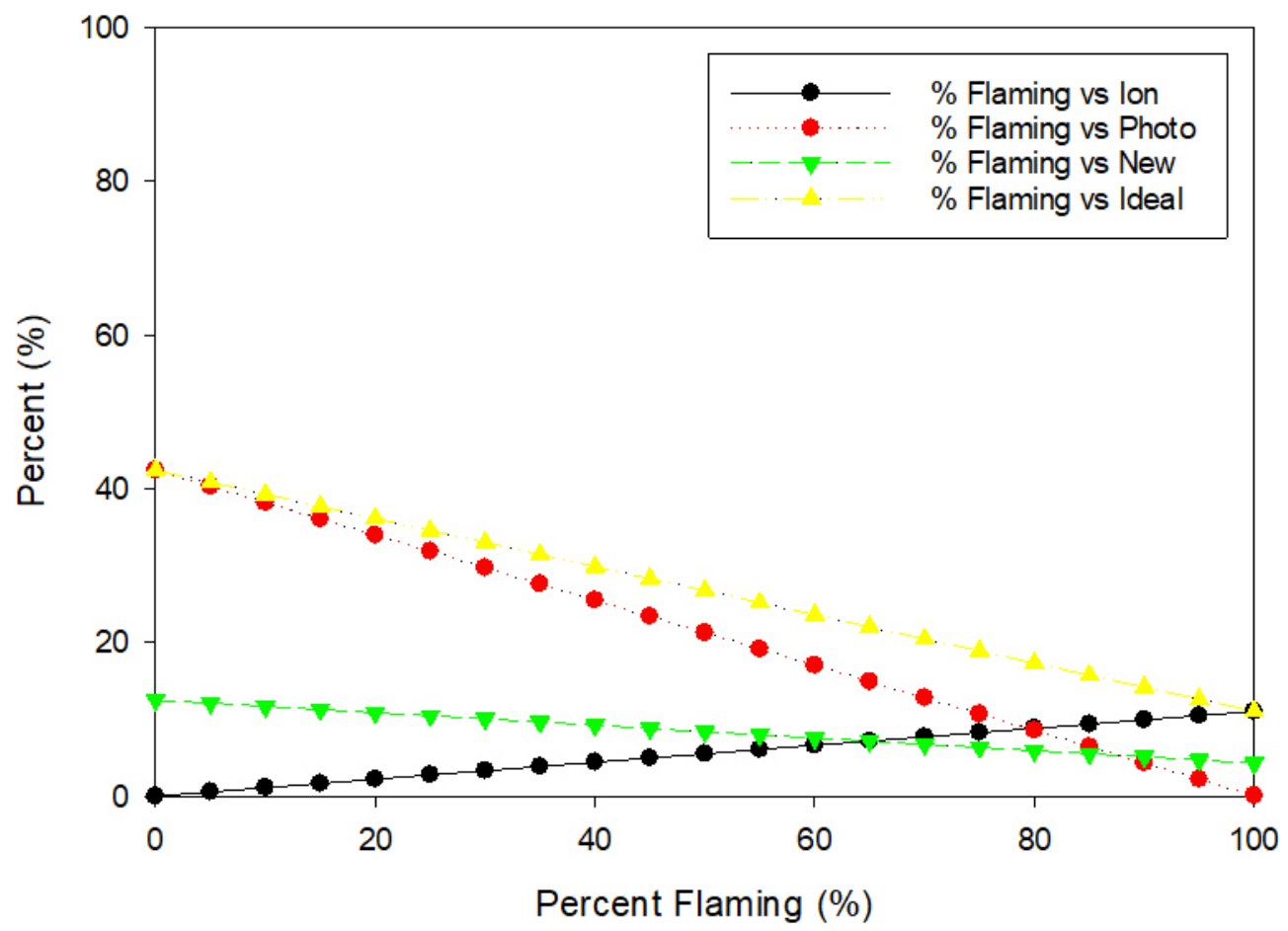

Figure 42 Overall performance of 4 alarms over range of highly toxic smoldering and flaming fires, FED = 0.3 and smoke considered, using the medium speed distribution.

Figure 43 shows that the performance of the alarms improves with the less strict tenability requirement $(\mathrm{FED}=1.0)$. It is still the case that both the new and ionization alarms are successful less than half the time for any combination of smoldering and flaming fires. Also, the photoelectric alarm has almost zero successes for the flaming fire, and the ionization alarm has zero successes for the smoldering fires. However, keep in mind that an FED of 1.0 only means that about half the people will die at that exposure, and more importantly in this analysis they are assumed to stay in place in the room that is the most toxic. Thus, this is not an attempt to predict actual results, rather to gauge relative performance of different alarm types under particular conditions. 


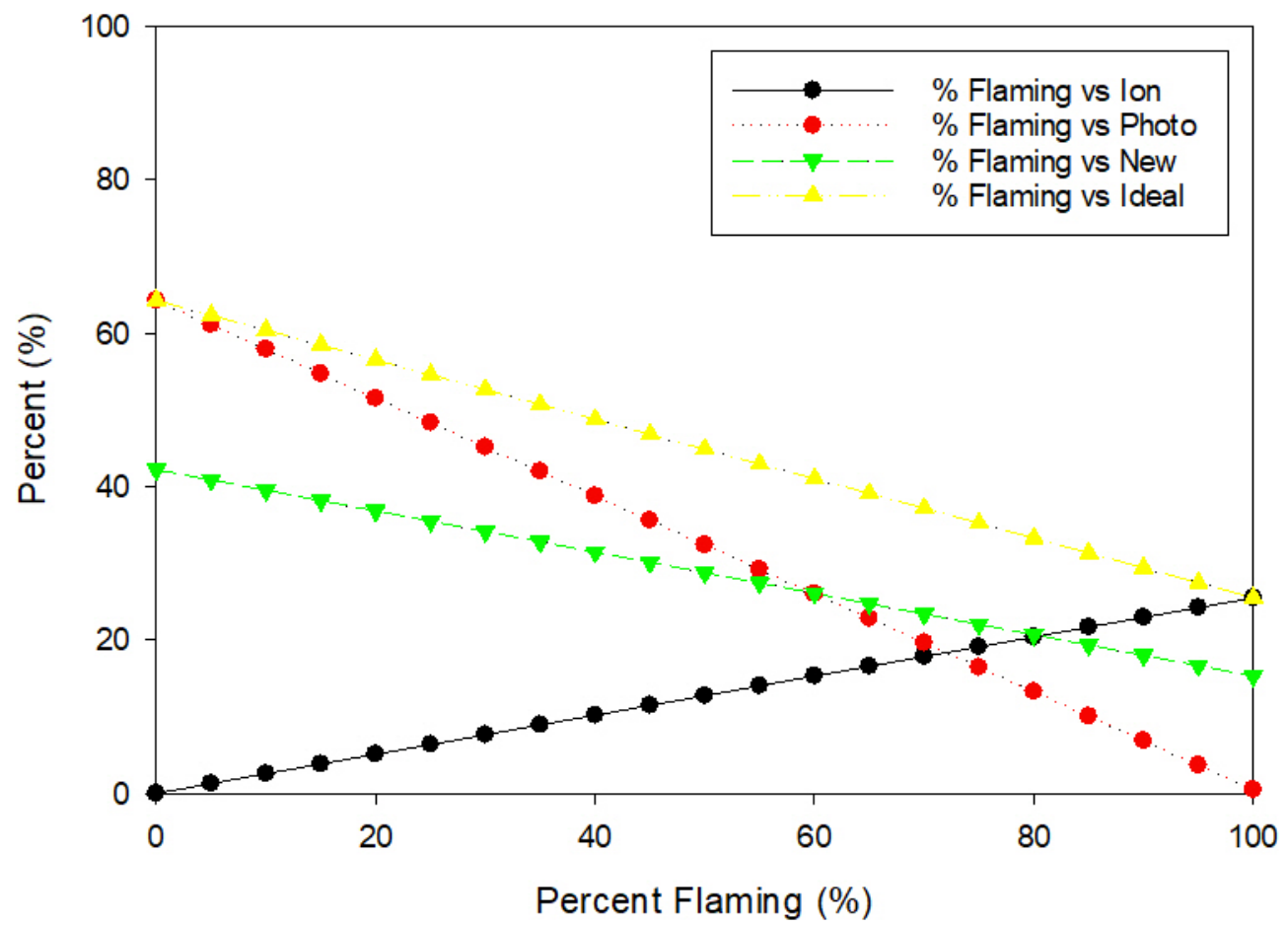

Figure 43 Overall performance of 4 alarms over a range of highly toxic smoldering and flaming fires, FED = 1.0 and smoke considered, using the medium speed distribution.

Also remember that this analysis considers a number of possible egress times. A requirement of $270 \mathrm{~s}$ after the alarm activates may be too restrictive. As Figure 44 shows, none of the alarms afforded occupants $270 \mathrm{~s}$ to evacuate before the first room reached an FED of 0.3 . In fact, the ionization ideal alarm only activates before any room reaches an FED of 0.3 in about half the cases. 


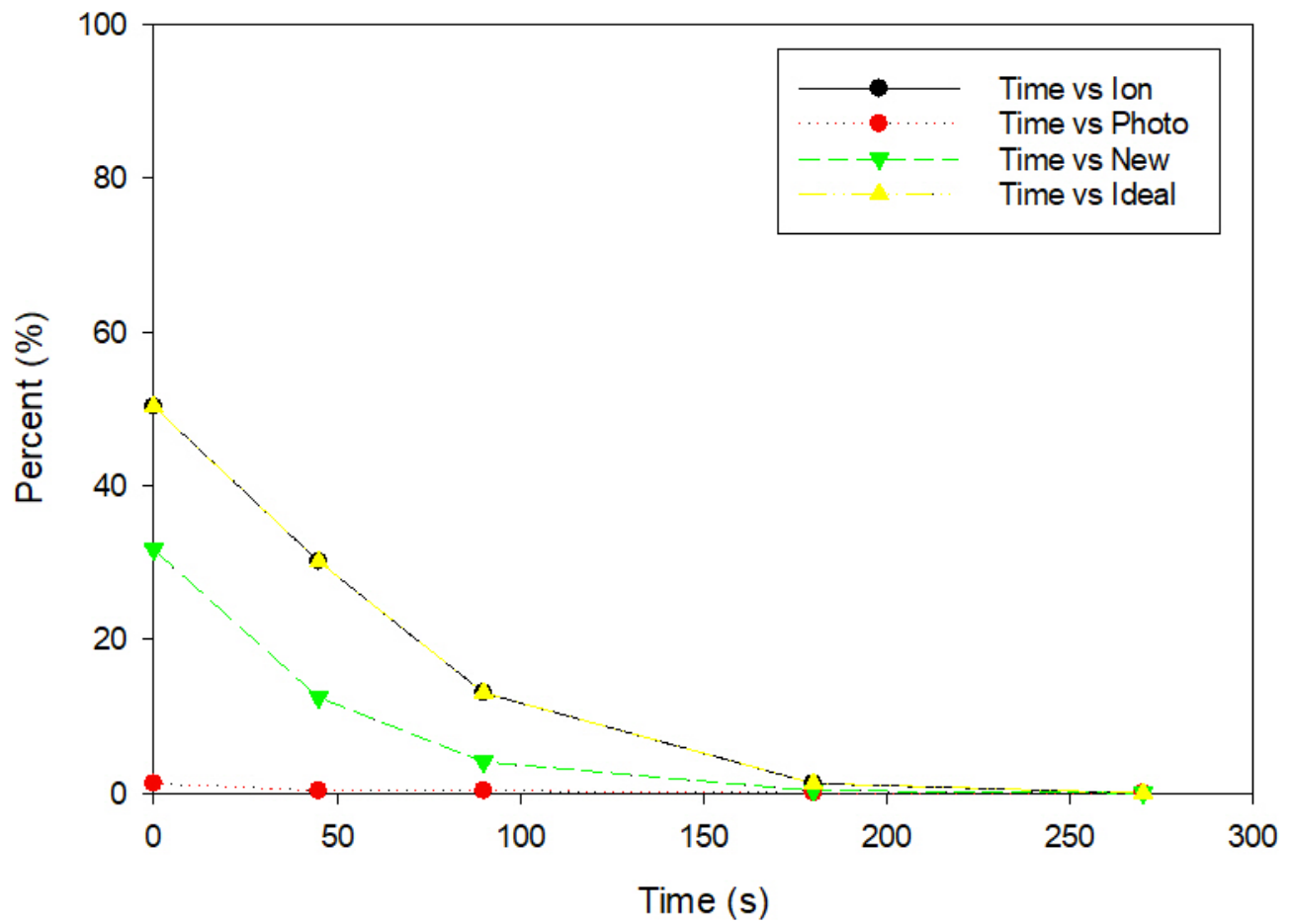

Figure 44 Percent of successful cases for egress for given time from high toxicity fires with $\mathrm{FED}=0.3$

\subsection{Uncertainty}

With a hazard analysis that has many different inputs, there are numerous potential sources of uncertainty. Some of the uncertainty was addressed by the using a Monte Carlo method and focusing on probabilistic answers. This section examines some additional sources of uncertainties, including modeling uncertainty and sensitivity of the results based on assumptions made for the analysis.

Considerable effort has been made in recent years to quantify the uncertainty in fire-growthrelated quantities $[63,64]$ and the accuracy and performance of computer-based models of fire behavior [64-68]. To place the discussion of model uncertainty in context, this section begins with an overview of the uncertainties for experimental measurements for detectionrelated model results (smoke concentration and alarm activation time) and tenability-related model results (gas concentration, temperature, and heat flux). Then, a summary of model uncertainty is presented for the same quantities of interest.

Since the model uncertainties are determined by comparing model calculations to experimental results, the accuracy of model predictions can only be quantified to the level of uncertainty of the associated quantities measured in experiments. For example, if the measurement of gas temperature in fire experiments is known to within $5 \%$, then at best we can quantify the accuracy of gas temperature in a model to within $5 \%$. Simplifying 
assumptions in the models add additional uncertainty, so that the model uncertainties are typically higher than corresponding experimental uncertainties.

For consistency with typically-reported model uncertainties, all discussion of uncertainties is presented as a relative standard uncertainty.

\subsubsection{Uncertainty for Quantities of Interest in Model Results}

Smoke Concentration: The systematic relative standard uncertainty for smoke concentration was reported to be $9 \%$ in Ref. [63]. The mean difference between replicate measurements was about $11 \%$. A combined relative standard uncertainty of $14 \%$ was suggested.

Smoke Alarm Activation Time: There is a single set of experiments with which to evaluate model predictions of smoke alarm activation time, the NIST Home Smoke Alarm Experiments [60]. The relative standard deviation of the normalized activation times for the NIST Home Smoke Alarm Experiments is 34 \%. Without more detailed information about the activation criteria, the models cannot predict the activation times more accurately than this value.

Temperature: In Ref. [63], Hamins et al. estimate the uncertainty of the various temperature measurements in fire experiments. Estimates of the combined relative standard uncertainty fall in a range between $2.5 \%$ and $7.5 \%$.

Heat Flux: The uncertainty associated with a heat flux measurement depends on many factors, including gauge characteristics, the calibration conditions and accuracy, as well as the incident flux modes (convective, radiative, conductive). Typically, the reported relative standard uncertainty of heat flux gauges varies from about $2.5 \%$ to $5 \%$, with the measurement uncertainty dominated by uncertainty in the calibration and repeatability of the

measurement. Repeatability of the various heat flux measurements in Ref. [63] was about 3.5 $\%$, on average. The combined relative stand uncertainty taking into account uncertainty in thermal radiation from the fire source was estimated to be $10 \%$.

Gas Species: From Ref. [63], relative standard uncertainty in the measured gas concentration was about $1.5 \%$ for both $\mathrm{O}_{2}$ depletion and $\mathrm{CO}_{2}$ concentration, and the difference between repeat measurements was about $1 \%$, on average, for both $\mathrm{O}_{2}$ depletion and $\mathrm{CO}_{2}$ concentration. The combined relative standard uncertainty taking into account uncertainty in a fuel's burning rate was estimated at $13 \%$.

\subsubsection{Modeling Uncertainty}

Details of verification and validation efforts with the CFAST model used for this study are available [68]. The validation calculations follow the techniques of McGrattan and Toman [69] that estimate the systematic bias and uncertainty of model calculations compared to experimental results for any number of comparisons. Table 16 presents a summary of CFAST accuracy (expressed as a relative systematic bias of the model results compared to experimental measurements. 
Table 16. Summary of CFAST bias and uncertainty for selected predicted results.

\begin{tabular}{|l|c|c|l|}
\hline Quantity & $\begin{array}{c}\text { Number of } \\
\text { Comparisons }\end{array}$ & Model Bias & $\begin{array}{l}\text { Experimental } \\
\text { Uncertainty }\end{array}$ \\
\hline Smoke Concentration & 15 & $3.43 \pm 0.68$ & 0.14 \\
\hline Smoke Alarm Activation & 142 & $0.56 \pm 0.51$ & 0.34 \\
\hline HGL Temperature & 297 & $1.09 \pm 0.32$ & $0.025-0.075$ \\
\hline Heat Flux & 244 & $0.97 \pm 0.61$ & 0.1 \\
\hline $\mathrm{CO}_{2}$ Concentration & 54 & $0.95 \pm 0.29$ & 0.13 \\
\hline $\mathrm{CO}$ Concentration & 24 & $1.17 \pm 0.64$ & 0.13 \\
\hline
\end{tabular}

Bias and uncertainty for model results are expressed as the ratio $(M-E) / E$ where $M$ is the predicted model value and $E$ is the measured experimental value. These are averaged over all the individual comparisons for a particular quantity.

Except for smoke concentration (and thus smoke alarm activation), results are typically predicted within $5 \%$ to $20 \%$ of experimental values. Higher model bias are seen for smoke concentration likely due to simplifications made for zone fire modeling, the lack of modeling of smoke deposition in fire models, and the assumption of instantaneous smoke alarm response in the model. Since the analysis conducted for this report was based on the relative performance of alarms, the higher uncertainties for smoke alarm performance should not impact the rank ordering of results in the report.

While the rank order should not be impacted by the modeling uncertainties, it is important to consider the impact of both the smoke concentration bias and the alarm activation bias. As expected, higher calculated smoke concentration leads to a correspondingly faster smoke alarm activation. Thus, tenability limits based on smoke obscuration would be reached earlier than predicted by the model, but this would, at least somewhat, be offset by faster than expected alarm activation times. Still, the high uncertainty in the calculations means that small differences in comparing different detection technologies should be discounted. Since the primary focus of this report is understanding the overall process for such comparisons, the comparisons are relative rather than absolute. Higher uncertainties associated with smoke concentration and smoke alarm activation time are acceptable in the context of the current study.

\subsubsection{Identifying Potential Issues with Uncertainty}

One way to investigate where uncertainty in inputs could have a serious impact on the analysis is to take the inputs and outputs of interest and model them with a statistical model to understand which inputs have the largest impact on the results 


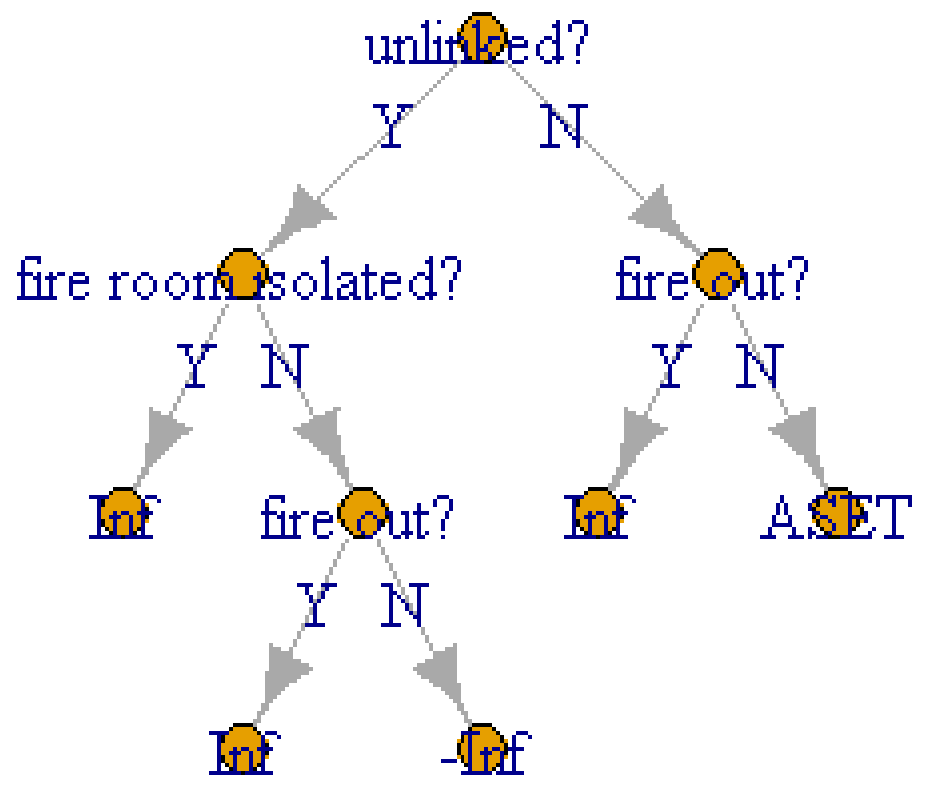

Figure 45. Multilayer model for regression of important analysis variables.

The way to think about this is as a multilayer model (see the Figure 45). If the fire room is unlinked from the room that contains a smoke alarm, then the alarm will never go off. That case is itself divided into two cases. In the first case the fire room is isolated from the rest of the house (we can think of this as having the doors to the room closed) and ASET time will be infinite. If it is not then either the fire will go out before hazardous conditions develop, resulting in an infinite ASET time, or it will not, resulting in a zero ASET time. In the latter case, this model assigns an ASET time of minus infinity because the alarm will never go off.

If the fire room is connected to the alarm room, the fire may go out before hazardous conditions develop. In this case, again, ASET time will be infinite. Otherwise we will need to develop a model for ASET time.

The likelihood that the fire room is isolated from the rest of the house and the likelihood that the fire room is unlinked from the alarm room are beyond the scope of this analysis. The remaining pieces of this model are analyzed below.

\subsubsection{Data}

Table 17 shows how many records for each fire type had the alarm unlinked from the fire room, how many of the remaining records had infinite ASET times (i.e., the fire went out before dangerous conditions developed), how many records had finite ASET times, and the total number of cases run for each fire type. 
Table 17. Number of test cases run for different categories of scenarios.

\begin{tabular}{|l|r|r|r|r|}
\hline Fire Type & Unlinked & Fire Out & Finite & Total \\
\hline flaming chair & 356 & 0 & 2319 & 2675 \\
\hline smoldering chair & 356 & 3 & 2435 & 2794 \\
\hline old mattress & 367 & 0 & 2426 & 2793 \\
\hline new mattress & 348 & 0 & 2390 & 2738 \\
\hline
\end{tabular}

There are two situations that arise with a finite ASET time. In most cases the model reported a positive value for ASET time, but in a few cases it reported an ASET time of zero. A zero value means that dangerous conditions were experienced before the smoke alarm activated. Table 18 lists the number of zero ASET times reported for each alarm and fire type, as well as the number of finite records for each fire type.

Table 18. Number of cases where untenable conditions were calculated before alarm activation.

\begin{tabular}{|l|c|c|c|c|}
\hline Fire Type & Ionization & Photoelectric & New & Total \\
\hline flaming chair & 0 & 0 & 0 & 2319 \\
\hline smoldering chair & 1 & 2 & 1 & 2435 \\
\hline old mattress & 0 & 0 & 0 & 2426 \\
\hline new mattress & 0 & 2 & 0 & 2390 \\
\hline
\end{tabular}

\subsubsection{Predictors of ASET time (conditional on being finite)}

ASET times provided by the model are centered at zero. That is, if dangerous conditions develop before the smoke alarm goes off, then the model returns an ASET time of zero. In order to analyze the censored model, we use a Tobit model.

We estimate the model for cases with finite ASET times, in Table 19. This determines what variables will help predict the ASET time for the fires in the model. This model estimates the model only for new type alarms. As discussed earlier, the times for the three types of alarms are highly correlated, so there is no need to estimate the model for all three alarm types. Since we are looking at finite ASET times, unlinked records need to be excluded from the analysis.

The following predictors were used in the analyses. 


\begin{tabular}{|l|l|}
\hline Variable & Description \\
\hline old.mattress & Dummy indicating whether this is an 'old mattress' type fire \\
\hline new.mattress & Dummy indicating whether this is a 'new mattress' type fire \\
\hline floor.area & Floor area of the house in square feet \\
\hline rooms & Number of rooms in the house \\
\hline links & Number of links between the fire room and the alarm room \\
\hline peak.hrr & Peak Heat release rate $(\mathrm{kW})$ \\
\hline flash & Dummy indicating whether flashover occurred \\
\hline
\end{tabular}

In addition to the individual predictors discussed above, this model considers their interactions with fire type. Essentially this allows us to estimate separate models for each fire type, instead of estimating a common model with different intercepts. In Table 19, the flaming fire values without a fire types listed represent the model for the flaming chair, while the flaming fire values with fire types listed represent the difference in parameter estimates for that fire type and the flaming chair.

For comparison, fire type was NOT interacted with all the other terms as well. The model with interactions was statistically different from the model without interactions. The likelihood-ratio [70] test had a p value $=0.0124$. Smaller values mean they are more likely to be different. A typical cutoff for statistical significance is 0.05implying. This implies that the models are different for different fire types.

Generally, increasing the floor area has an ambiguous effect on the amount of time people have to evacuate. Increasing the number of rooms increases the amount of time people have to evacuate. Increasing the number of links between the fire and the alarm decreased the . ASET. An increase in peak HRR decreases the amount of time people had to evacuate. Unlike peak HRR flashover does not have a statistically significant effect. The initial linear growth rate was not statistically significant in this model.

As discussed in Section 4.1, exclusion of cases because the fire room is unlinked from the alarm room has the potential to bias the results since being unlinked is not independent of number of rooms. These results suggest that if such a bias in the number of rooms exists then, since the number of rooms is positively correlated with available evacuation times, the bias will increase the amount of time available for evacuation, meaning a higher probability of success. 
Table 19. Regression analysis of analysis predictors with ASET for flaming and smoldering fires, when the fire has a finite ASET (when fire room is connected to alarm room).

\begin{tabular}{|c|c|c|c|c|c|c|}
\hline Term & Paramet & er Estimate & $\begin{array}{l}\text { Marginal } \\
\text { Effect }\end{array}$ & $t$ statistic & Prob. & flag \\
\hline \multicolumn{7}{|c|}{ Flaming Fires } \\
\hline (Intercept) & 372.57 & \pm 20.22 & 372.6 & 36.12 & 0 & $* * *$ \\
\hline floor.area & -0.0089 & \pm 0.0066 & -8.57 & -2.64 & 0.008 & $* *$ \\
\hline rooms & 5.53 & \pm 3.64 & 9.73 & 2.98 & 0.003 & $* *$ \\
\hline links & -105.99 & \pm 6.71 & -71.16 & -30.98 & 0 & $* * *$ \\
\hline peak.hrr & -0.0010 & \pm 0.0071 & -1.34 & -0.27 & 0.789 & \\
\hline flash & -3.39 & \pm 14.28 & -3.39 & -0.47 & 0.642 & \\
\hline old.mattress & 33.16 & \pm 34.01 & 33.16 & 1.91 & 0.056 & . \\
\hline old.mattress:floor.area & 0.0037 & \pm 0.0091 & 3.93 & 0.81 & 0.42 & \\
\hline old.mattress:rooms & -1.72 & \pm 5.05 & -5.33 & -0.67 & 0.504 & \\
\hline old.mattress:links & -12.9 & \pm 9.60 & -10.64 & -2.63 & 0.008 & $* *$ \\
\hline old.mattress:peak.hrr & -0.0050 & \pm 0.0093 & -8.48 & -1.06 & 0.291 & \\
\hline old.mattress:flash & 11.43 & \pm 17.37 & 11.43 & 1.29 & 0.197 & \\
\hline new.mattress & 17.55 & \pm 28.82 & 17.55 & 1.19 & 0.233 & \\
\hline new.mattress:floor.area & 0.0082 & \pm 0.0092 & 8.47 & 1.73 & 0.083 & . \\
\hline new.mattress:rooms & 3.06 & \pm 5.11 & 9.34 & 1.17 & 0.24 & \\
\hline new.mattress:links & -11.99 & \pm 9.34 & -9.98 & -2.52 & 0.012 & $*$ \\
\hline new.mattress:peak.hrr & -0.0028 & \pm 0.0235 & -0.7 & -0.23 & 0.818 & \\
\hline new.mattress:flash & -19.71 & \pm 77.14 & -19.71 & -0.5 & 0.617 & \\
\hline \multicolumn{7}{|c|}{ Smoldering Fires } \\
\hline (Intercept) & 2509.62 & \pm 115.48 & 2509.62 & 42.59 & 0.0000 & $* * *$ \\
\hline floor.area & 0.0783 & \pm 0.0379 & 74.74 & 4.05 & 0.0001 & $* * *$ \\
\hline rooms & 46.71 & \pm 20.95 & 81.63 & 4.37 & 0.0000 & $* * *$ \\
\hline links & -565.18 & \pm 37.36 & -386.61 & -29.65 & 0.0000 & $* * *$ \\
\hline peak.hrr & -0.0134 & \pm 0.0413 & -8.58 & -0.64 & 0.5236 & \\
\hline flash & 6.44 & \pm 83.3 & 6.44 & 0.21 & 0.83 & \\
\hline
\end{tabular}

"Prob." is the probability that the true value of the parameter is zero. "Flag” is "***” if probability is less than $0.1 \%$, “**” if probability is less than $1 \%$, “*” if probability is less than $5 \%$, and “.” If probability is less than $10 \%$. The \pm values are $95 \%$ confidence limits. 


\section{Discussion}

Since the overriding objective of this project is to develop a better understanding of the process of a quantitative fire hazard analysis, a number of aspects of the analysis are discussed. In addition to a presentation of the actual analysis, with the aspects discussed are: the implications on needed data for future analyses, the research needs in residential evacuation to provide more than just a crude estimate of the time necessary to escape safety from a fire, and the needs for additional information on residential floorplans and fire scenarios to better tie the results of an analysis to realistic fire exposures. Current understanding of modeling and statistical analysis of modeling results is also discussed.

\subsection{Analysis of Smoke Alarms}

While the analysis of smoke alarm performance was a vehicle to allow quantitative study of the process of fire hazard analysis, smoke alarm performance is obviously a part of the results. For that reason, we present a discussion of some of the results.

With any kind of analysis, the most desirable result is a clear and unambiguous result: pass or fail; yes or no; better or worse. With any design, every task that has a straightforward answer makes the whole project that much easier. For this analysis there is not a single straightforward answer. If the question is what alarm will perform the best across all scenarios, then the answer is the new alarms. If there is some knowledge that implies fires are more likely to be mostly flaming or mostly smoldering, then either the ionization or photoelectric alarms, respectively, would be the better choice.

It is clearly the case that the ideal alarm, if it were feasible, would be the best performing alarm for all conditions. However, from the sensitivity analysis it seems clear that the new alarm does not have to achieve the performance of the ideal alarm to be the best choice. If assumptions about the average performance are pessimistic, it could be the case that the new alarms will clearly be the best for all options.

One important result is that the slower the fire reaches hazardous conditions, the better all the alarms performed, especially the new alarm which performed much closer to the ideal in the slower developing fires. The question is how often are fires slow to develop and how often are they fast. The answer is outside of the data that was available for this study.

One of the problems with the analysis is lack of a model of occupant egress. Having a more accurate measure of each individual fire egress case would eliminate some of the uncertainty in the analysis. While it might seem that the most conservative answer is the best, in reality the expected improvement based on the analysis might not be achieved, and the additional costs may not be warranted.

In terms of an alarm allowing time to investigate and fight the fire, the performance of the new and ionization alarm was about the same. The new alarm was somewhat better on the smoldering fires, and the ionization was a little better on the flaming fires. The photoelectric alarms did not do nearly as well as the other two on the flaming fires. Here, time was shown to be a very important factor. Because the flaming fires transitioned to vigorous burning 
quickly, the ionization alarm allowed occupants to find a smoldering fire with a high probability of putting it out more often than it did for a flaming fire, that it is better at detecting.

For reasons of liability if nothing else, safety officials may understandably be reluctant to even suggest that occupants attempt to fight the fire and may only want to consider technology that improves evacuation. However, consider two different technologies that reduce the total HRR of a product, A and B. Suppose A is completely new technology that reduces the total HRR by a factor of 10 . However, items that do ignite are very fragile and generally crumble when occupants try to put out the fire spreading hot embers everywhere. The B technology does not reduce the total HRR nearly as much, but the fires are fairly easy to extinguish without spreading the fires. In a Monte Carlo analysis that only considers evacuation A would be the better option but in reality, A might actually increase fire casualties if the fires are hard to extinguish. Not considering the impact that a new technology, in our case the new alarms, has on all occupant behavior, including fighting the fire, may lead to significant under or over prediction of the overall benefits the technology offers.

Of course, it is important to note that improved detection may not be the only desired advantage of the new alarms. New alarms may also be intended to significantly reduce false alarms. False alarms can lead to occupants disabling the fire alarms. In 2016 NFIRS data where the cause of alarm failure was determined, $42.8 \%$ of failures were due to "the battery missing or disconnected." Still, a quantitative analysis of performance for different designs can provide a better understanding not only of the performance per se, but also of the impact of chosen tradeoffs for alternative designs.

\subsection{Data}

Available data is certainly important for a process as complex as residential fires. The following three sections deal in part with specific aspects of the data problem. This section will deal broadly with the general issues with the data currently available and what may be beyond reach for the foreseeable future.

Early in the project, an attempt to predict some aspects of the NFIRS data was considered. How accurately the Monte Carlo process was in predicting actual fire statistics could be used as an indication of how close the Monte Carlo process could predict the actual impact of new technology. One possible fire statistic was how often the fire spread beyond the room of origin, which could be assumed as describing when the room reached flashover.

However, it soon became apparent that there was so much needed data that was not available that it would be impossible to model the fire growth for even one particular room. Issues included but were not limited to the geometry of the room and the ventilation conditions. Often before a room reaches flashover, a second item and possibly more items become involved. The ignition of a second item is very dependent on what the item is and the location of the second item with respect to the initial burning item. Furthermore, the HRR of the second or third item is important to the possibility of flashing over the room and how fast that happens. 
Additionally, the tendency of fire to flashover certain rooms and geometries faster than other geometries requires a fairly detailed distribution of the compartment geometries, ventilation, and contents. For example, smaller rooms need a smaller fire to flashover than a large room requires. More ventilation means a larger fire is needed to flashover the same sized room. That means that having a distribution of the size of the rooms and a distribution of the number of doors into the rooms is not sufficient to predict flashover. It is also important to know if there a correlation between the number of doors in the living room and the room size or between the number of doors in the living room and the total floor area. Variation in fuel loading as a function of room size could also impact the results. Few of these data are available.

Of course, occupant normal behavior in the house also has to be considered. Suppose in one house the living room is very central to the floorplan resulting in a lot of through traffic. In another house, the living room is off to one side of the house with limited through traffic. At least during daytime hours, a room with more through traffic might result in more fires being extinguished before they can grow to flashover compared to a room with less through traffic. Or the fact that a room is not frequently used might mean it is much less likely to ever have a fire.

The exercise of trying to predict the rate of flashovers showed that quantitatively predicting the actual impact of different fire technologies is not something that will realistically be accomplished soon. What can be done is to develop measures of the relative impact of different technologies. Of course, this is not as satisfying a result because it means a certain amount of expert judgement is still needed to evaluate new technologies.

\subsection{Residential Evacuation}

One part of estimating the risk and quantifying any improvement of risk in residential fires is accurately representing people's actions when faced with a fire. A discussion of some of the behaviors that have been noted will be made as well as recommendations for the kind of model that is included below.

In terms of designing the fire safety systems of a building, significant efforts have focused on calculating the available safe egress time, ASET. Models like FDS [71] and CFAST [72] have been under constant development for decades and have begun tackling the difficult issue of verification and validation [73]. On the RSET side of the equation there are a significant number of egress models available; however few, if any, are designed for residential evacuations [74].

Babrauskas, Fleming, and Russell [75] argue that the RSET part of the equation is problematic since it assumes that people act in a fairly rigid "robotic" fashion. While it may be reasonable to make the simplifying assumptions of RSET for commercial buildings where crowd dynamics are a particularly important part of the evacuation, a simple definition of RSET is not particularly useful for individuals in residential egress. This view is supported by a NFPA survey reported by Ahrens [76] that of people that heard the fire alarm in their residence at least once in the last 12 months, only $8 \%$ thought it indicated there was a fire 
and that they should evacuate. In the survey's most popular answer, $24 \%$ said their first thought was that food had burned. For $8 \%$ of the respondents, the first thought was to investigate the alarm, the same number as who thought to evacuate. Wood's and Bryan's analyses of first three actions taken during residential evacuations further bolster the argument that RSET is too a simplistic way to look at residential evacuation. Wood's analysis [28] of the first three actions of evacuation in UK residences found the most popular first action was "some fire-fighting action," and the third most likely first action was "investigate fire." Bryan found that the most likely first two actions were "notified others" and "searched for fire" [29]. Both reports show that there is a relatively high probability that occupants will not only investigate fire cues but try to mitigate any fire that is discovered.

Now consider someone that does investigate a fire thinking that it is something burning on the stove and discovers that there is a fire that originated on the stove. If that person decides to attempt to fight the fire and only decides to evacuate once he or she loses control of the fire, what is the RSET? Should the calculations include scenarios where the resident would fight the fire but be unsuccessful?

In addition, both Babrauskas et. al. [75] and Hall [37] report that if people try to escape, at least early in the fire, they will succeed. This strongly suggests that the risk involved with fires in residential occupancies is largely based on behavior and less on movement. The typical definition of RSET is mostly a based on movement time during egress.

Thompson and Wales [42] found that $50 \%$ of the injuries reported in their study were from attempts to fight the fire. In the investigations of actual fires with fatalities, Bryan and DeNinno [31], and Pauls [35] found that the fatalities were due to long delays in starting evacuations. Are we to assume that fighting the fire and the long delays in starting evacuations are part of a required egress time? While this is a limited set of fire investigations and could suffer from selection bias, it is important to note that it was not the fire that directly killed anyone. It was that time was given for smoke to spread and fill parts of evacuation paths so that occupants could not effectively escape. As noted, Jin and Yamada [51] found that traveling through a smoke filled hallway slows travel speed and impairs cognition.

Finally, in studies of building evacuations, Proulx [32, 34] and Proulx and Fahey [33] found there was a strong correlation between total building evacuation and the length of time it took for the population's recognition and evacuation decision. This makes the point that the preevacuation actions are a very important factor in evacuation time and ultimately occupant safety.

Kuligowski [77, 78] and Kuligowski et. al. [79] have made the argument that in current evacuation models, the inclusion of pre-evacuation behavior in egress simulations is primarily user-driven (via inputs). However, as investigations and fire drills demonstrate, it is the pre-evacuation time that can have the largest impact on total egress time as well as having the largest uncertainty. Therefore, pre-evacuation time also has the greatest impact on the likelihood of a successful egress outcome.

Residential egress, especially in one- and two- family dwellings is dominated by individual behavior. When an alarm sounds people do not simply react by preparing to egress and then exiting. Often they investigate the fire, and if they discover a fire, they may attempt to put out 
the fire [28, 29, 76]. There is also the rescue behavior of caregivers, especially parents, where the individual engages in potentially risky behavior in order to save others [37]. A welldesigned residential egress model would have to take into account these behaviors as well as the statistical nature of any analysis.

Even with better statistics than are currently available, it is likely that in any given situation a given demographic group will have several probable behaviors. Given the range of actions, any analysis of even a single scenario could only be reported as statistical probability of people escaping, and a residential egress model should be built around supplying that statistical analysis.

\subsubsection{Occupants of Particular Concern}

There are a number of groups that need special attention in considering evacuations and risk. Residential evacuation models would need to be able to account for each of these groups when generating evacuation times.

Sleepers: This is a group that has been of particular interest to the research community. Six of the experimental studies [43, 45-49] reviewed focused on the conditions that will wake sleepers or some subgroups of sleepers. Bruck and Ball's [38] analysis of the risk factors that confound with sleep found a number of factors that increase the risk when sleeping. They also found that typical alarms (at the time of the research) would not wake at risk groups.

Young children: Bruck and Ball [38] found that one of the risk groups when people are sleeping are children. Bruck's experiments [46] showed that alarms may not be effective in waking children as old as 15 years of age. Harpur, Boyce, and McConnell [41] found that children under the age of 5 are at greater risk of being injured or killed in a fire. So, some children are not just a risk factor when asleep but also at higher risk when awake.

Mobility challenged: There was not a lot found in the fire research literature about the mobility challenged and residential evacuations. Pearson and Joost [44] did look at how being bound to a wheelchair or blindness affected egress, and Kady and Davis [50] looked at the potential impact of being overweight on crawling. However, it is clear that any challenge that increases egress time or requires assistance is going to be a special concern in trying to model residential egress.

Older Adults: Not every older adult is at greater risk than the average person, but older adults have higher rates of a variety of issues that may interfere with their ability to self-evacuate. So, the group has to be considered at higher risk. Older adults was the final risk factor identified by Bruck and Ball [38].

Parents and caregivers of those that would need or might need assistance: There is little discussion of this group in the egress articles that were reviewed. Bruck [46] found that $100 \%$ of parents awoke for the alarms. Anecdotal evidence suggests that parents and other caregivers can and do put their own life at great risk to save others. Hall [37] notes that the rate of deaths to injuries for people going to rescue another is higher than for people going to fight the fire. Hall offers one interpretation which is 
that people will put themselves at greater risk when trying to save another than when simply fighting the fire. Regardless of the reason, it is clear that people with responsibilities for others may be at a greater risk than people that only have to worry about themselves.

Those who are intoxicated or on drugs that inhibit their ability to evacuate: Two of the groups that Bruck and Ball [38] found to be at increased risk when asleep are those that took sleeping tablets and those that are intoxicated on alcohol. Ball and Bruck [48] found that a blood alcohol level as low as 0.05 would slow the response of a sleeping person to an alarm. Clearly consuming larger quantities of alcohol, sleeping pills or other depressants will inhibit a person's ability to respond to a fire alarm or other fire cues.

\subsection{Residential Floor Plans}

The AHS serves as a source of information on residences, but it lacks certain important data. There is no information on actual floorplans of residences, and therefore no basis on which to develop a standard floorplan.

In the review section, each set of researchers made their own decision about a floorplan for their scenario. A number of researchers choose to model a part of a house instead of a full residential floor plan with the assumption being that the rest of the house is sufficiently removed from the rooms of interest that they don't need to be modeled. Bukowski [4] used a three room section of a house to look at the fire hazard of a furniture fire. Bruns [20] also used a three room section of a residence. Ohlemiller and Gann [15] used the same geometry as Bukowski [4] with the addition of a fourth room that represented the rest of the house. Others have used full floorplans. Three studies $[5,7,16]$ used full ranch style houses. Peacock et al. [16] also considered a hotel room and an office. Finally a number of studies [8, $10,14,19]$ used a single room when only the room of fire origin was of interest. They all make the same assumption that the rest of the building will not have a significant impact on the values of interest in their study. None of the studies did any analyses to show the extent to which their assumption was valid.

While it may be the case that the rest of the house does not impact what occurs in the section of interest, for egress calculations there is the question of whether people actually get out before the fire becomes untenable. Furthermore, there are some obvious architectural design features being ignored in all of these analyses, for example multi-level homes. Even more importantly, most of these studies make no attempt to look at the impact of residential egress. Even the studies $[6,7]$ that do consider egress do so in a limited set of conditions.

If, as we expect, residential egress is deemed to be essential to understanding the fire problem and the impact of fire safety technology, the study of full floor plans is necessary. If floor plans are important, researchers will need access to information about floorplans. There are many questions to be answered including how much variability is needed to truly capture the full impact of the floorplan on a scenario under study.

One possible solution is to come up with a set of housing floor plans that represent the variability of housing designs. It would be important to include a distribution of housing plans. For example, one variable is what percentage of houses have multiple floors. While 
such a set would not capture all the variability, it should be able to sufficiently represent possible designs to capture major problems that one or more designs may have.

\subsection{Fire Scenarios}

Of all the data related issues, the definitions of fire scenarios are key. Babrauskas and Peacock noted that HRR is the single most important variable in fire hazard [80]. In addition, the type of ignition and the distribution of the types of ignition are seen as important. As shown throughout chapter 4, the greater the fraction of smoldering fires, the better the photoelectric alarms performed, while the ionization alarms did much better for fires that started by flaming ignition. We were able to get a clear picture of the impact of the distribution of smoldering and flaming fires because we varied that parameter.

The linear ramps for both the flaming and smoldering fires were taken from distributions based on experimental data. Those distributions were based on a particular scenario, the fire starting on the leading edge of the seat cushion[58]. The distributions were also finite, meaning fires did not transition to vigorous burning very quickly, say less than $60 \mathrm{~s}$. Suppose faster transitioning fires were included in the analysis. From sections 4.4 .2 and 4.5 where the available time was reduced due to the inclusion of a smoke criteria or the significant production of HCN, the performance of the new alarms relative to the ionization alarms for flaming fires and the photoelectric alarms for smoldering fires was reduced. It is reasonable to extrapolate that greatly shortening the linear ramps would hurt the relative performance of the new alarms the same way. Although if the smoldering ramps are shortened to $60 \mathrm{~s}$ it is likely that the fire would transition to flaming before the photoelectric alarms activate and the new alarms' relative performance compared to the photoelectric alarms would improve, which further proves the point.

Considering all the variables and the different types of fire scenarios that are documented in the NFIRS database, it seems unlikely that a universal set of fire scenarios could be developed. For specific applications of alarms and alarm locations, a standard set of scenarios could be developed. However, for other technologies like barrier fabrics and fire retardants or even fire suppression devices, the fire scenarios that need to be considered would be much more application specific.

What are needed are guidelines to the variables and scenarios that need to be considered in an analysis. For specific items being tested, there needs to be some guidance in determining how likely a second item is to be ignited. Guidance needs to be developed in how to account for variability. Should the variability that was observed be used, as was done here, or should a wider range be used to account for limitations in available experimental data? These are questions that need to be addressed.

Even if the modeling of occupant behaviors is outside of the analysis tools available, some attempt to account for those interactions needs to be made. For example, in this analysis we looked at the impact of the new alarms on having time to investigate and find the fire, which included some idea about what fraction of the fires occupants have a greater probability of successfully putting out. 
Hopefully, as this kind of Monte Carlo modeling becomes more widespread, experience and research will help develop the needed guidelines.

\subsection{Modeling}

As stated in the introduction, the long-range goals of this project are to provide tools to help make Monte Carlo analyses easier to do. The term modeling means here the process of creating a large number of cases and running them in a computational tool, in our case, CFAST. There is also analysis of results, but most of that discussion will be in the next section.

First we will discuss some overall lessons from the process of creating a large number of cases, running them in a model and what to do with the output. Next will be a discussion of some specific issues with varying inputs to attempt to understand what the most useful inputs are in the development of any tools.

In section 3.4 the method used to generate the data was described. A program was developed in Python that generated the specific input files for CFAST, ran the model, and then processed the output to generate a single comma delimited file for all the runs. There was an elegancy to the solution. The program generated each case, ran CFAST, and collected the identified data in sequence so that a large number of input files and output files did not have to be stored on disk. While it was obvious beforehand that the program would not be easily generalized, the process seemed promising, and it did generate 57500 cases.

However, there were problems. After generating the first 57500 cases, it was realized that one of the important tenability criteria that has been considered in the past was the smoke concentration, but it had not been recorded in our data set. Because the original output from all the runs of CFAST had been discarded, it was not possible to go back and collect smoke data. So, the 57500 cases could be used for the alarm activation and fire investigation activities analyses, but that set of cases could not be used for the tenability analysis needed to estimate successful egress.

Our experience also pointed to another scenario that did not happen but could happen in other analyses. While our analysis provided insights into the issues with smoke alarms, there were not any true anomalies to be explained. Had there been, having all the calculated data available may have sped up the process of understanding such results. So, one of the important lessons is that, if possible, when doing a Monte Carlo analysis, keep all the data available at least until the analysis is complete, rather than a priori deciding what data to keep from simulation results. Part of the data needed for analysis is the input files used to generate the output.

Another lesson learned is that it is best not to assume too much about the platform the analysis is to be run on. While the Python code and CFAST were run successfully on Windows, OSX and Unix systems, the best way to organize the running of CFAST was not the same for all platforms. For a large Unix cluster with many parallel computational nodes, it seems likely that parallel processing should be used to take full advantage of the available computational capability. It might be best to separate the running of the fire model from 
creating the input files and the post processing, so that the user can make the best use of the resources that are available.

In terms of a tool to create or help create the large number of input files needed to do a Monte Carlo analysis, the lesson is that there are a lot of parameters that can be varied. Moreover, different variables have different types of distributions. For example, the number of rooms is a discrete variable that can only take on whole numbers. Other variables can be thought of as discrete such as material. Suppose as part of an analysis, the effect of different wall materials was to be investigated. It might be the case that the analysis considers gypsum board, wood, and concrete. In that case the wall material would be a discrete random variable. If, on the other hand, the focus was to look at the impact of the density of the concrete walls on the upper layer temperature, the distribution might be a continuous random variable.

There are also variables that affect time histories like the HRR curve. The number of points can change; the shape of the curve can change. It is not trivial to describe the variability of the curve, which leads to simplifying the types of shapes that can be used. That begs the question of what shape or shapes should be used. Any choice that is made can limit the usefulness of the tool.

It would seem obvious that any tool that is designed to generate random files for fire analysis should be able to vary the fire over a significant range of curves. However, it is not at all clear what the best parameters to vary are. It is not clear how to best go about selecting the parameters that should be available to be varied first. Unfortunately, no single analysis is likely to provide that much help in answering that question in general.

\subsection{Analysis of Results}

The analysis of all the data led to a number of realizations. The first is that with so much data, there are a range of questions that can be addressed, and any tools to assist in the analysis would need to be flexible to help answer all questions. While the primary answers for this analysis came from collecting data into probabilities of success and failures, that was not the only important data analysis tools used.

For example, we used histograms to understand that in the original 57500 cases, CFAST had problems running cases of the old mattress fires more than any other fire type. This turned out not to be a problem since we partitioned the data by fire type, and there were more than enough of each fire type to generate good statistics, but it was an important issue. If we had tried to partition the data in another fashion, the under representation of the old mattress fires might have biased the results. In other analysis it is conceivable that questions being asked might need a histogram for the answer. For example, the question: what are the distribution of peak temperatures a structure might face for a given set of fires?

A second realization has been mentioned already in section 5.6. Keep all the data if at all possible. There are several issues with keeping all the data, for example, having available storage space. To see another, suppose that we had kept all 57500 sets of outputs. Consider the effort that would have gone into looking through 57500 output files manually to find the 
time the smoke first reached an optical density of $0.25 \mathrm{~m}^{-1}$ in any room in the simulation. Obviously, that is a task where it would be desirable to automate part or all of the task.

Initially the most useful tools to help the analysis process would be tools that could collect data from a large number of output files and group the data together in a new file for analysis. However, collecting data from just the output files is not enough. It was important for our analysis to be able to relate inputs to outputs, meaning that any analysis might need to be able to relate input data to output data. This means that we would need tools that could read and collect information from both the input files and the corresponding output files.

Clearly, any effort to develop tools for the analysis part of a Monte Carlo process should focus initially on collecting and grouping data. The most basic tool for a Monte Carlo analysis done with CFAST would be to collect identified columns of data from a large number of files and group it in a single output file.

Grouping data from input files can be more difficult. Most of the input data that was useful was basically meta data or processed related to the model inputs and not data directly in the input files. For example, fires were grouped according to the type of fire the distributions came from to define the fire (such as "smoldering chair", "flaming chair", or "flaming mattress"). It certainly would not be easy and may not be possible to determine which fire was being used for a specific scenario except for the smoldering chair. Another important bit of input data that was important was if there was a path for smoke to travel form the fire to the alarms.

In order to collect the most useful data from the input files it might be required that any tool to build collections of input files would be able to include meta data in each input file. This would certainly add a level of sophistication to the tool, and it seems likely that it would be needed for an analysis of a problem.

\subsection{Uncertainty and Sensitivity in Predictions and Results}

Uncertainty and sensitivity are not the same thing, but they are related and can be combined to provide a better understanding of the results of a Monte Carlo study. For example, suppose that input $\mathrm{A}$ is known to within $10 \%$, but the results of the analysis vary by less than $1 \%$ with a $10 \%$ change in $\mathrm{A}$. The finding is that the analysis is not overly impacted by the uncertainty in A.

Most of the uncertainty in this analysis was due to a lack of data. The biggest lack of data is for the evacuation process in residential structures, especially but not limited to how occupants interact with the fire. As has been stated a number of times, there is a strong need for a model of residential egress.

Some uncertainty can be dealt with in the analysis. It is not clear what fraction of fires are smoldering and what fraction are flaming fires, but we looked at the results over a range of possible distributions of fires to give a fuller understanding of the impact of new alarms. 
Due to the uncertainty in how new alarms will function, we looked at the sensitivity of the results to changes in the performance. This is the most straightforward way to examine both the uncertainty and the sensitivity of the analysis, but it is very data intensive. Therefore, it is prohibitive to explore the sensitivity of a significant number of uncertain variables that way. It would be possible to examine a larger number of variables if fewer simulations were used for each variable examined, instead of using the full number of simulations, in our case 10 000. Perhaps as little as a few hundred cases could give insight into the order of magnitude of changes, but there will always be the possibility of being misled by the variability from small sample sizes.

One way to look for the sensitivity and the potential impact of uncertainties is by making use of statistical models relating the inputs to the outputs. This is how we examined the sensitivity of our analysis and made qualitative estimates of the impact of certain uncertainties. Within the confines of the data, these models can give significant insight into the analysis. It is also a more efficient way to explore the sensitivity of the analysis. While it takes knowledge of a sophisticated statistics package to generate the analysis, it was significantly less time than generating the actual data.

For the uncertainty and sensitivity analysis, little is needed in terms of the type of tools that would be useful to develop for the Monte Carlo process. General purpose statistical tools already exist to do this type of analysis. Tools that allow collecting and grouping data from output files as well as input files are the most pressing need for this analysis.

\section{Conclusions and Future Research Needs}

There are many fire safety technologies that can be pursued to reduce the loss of life and property. One problem is to determine which technologies have the greatest potential and how much each technology can improve safety. When attempting to address this issue one technique that has been used a number of times, as discussed in section 2.1, is Monte Carlo analysis.

To help make Monte Carlo analysis a more widely used process NIST has embarked on a project to create tools and guidelines to make the process more tractable. To identify areas where the greatest impact can be made, we decided to perform a sample analysis of the impact of new smoke alarm standards, ANSI/UL 217 2015, on measures of fire safety. After doing this analysis, some conclusions and recommendations for future research are apparent.

Firstly, Monte Carlo analysis is an important and powerful tool that can give a great deal of insight. Monte Carlo analysis should be done more often then it appears is current practice. There are a large number of inputs into any fire analysis. It is not realistic to assume that expert opinion can always determine the impact of all variables. A Monte Carlo analysis, when properly done, can identify parameters and relationships in the scenarios being considered that might otherwise be overlooked.

As an example of the insights that can be gained, our analysis of the impact of the new smoke alarm standards showed that for fires that become hazardous more slowly, typically 
smoldering fires, the advantage of the new detectors orver the ion detectros appeared to be somewhat limited. As the speed of conditions becoming hazardous increased, typically flaming fires, the advantage of the new alarm over the ionization and photoelectric alarm appeared to increase to some extent. However, if it is known that the fire threats are mostly from smoldering fires or mostly from flaming fires, the type of detector technology can be tailored to anticipate the fire.. While the impact of sensitivity may seem obvious at the start of the project, we did not anticipate where the limits were on the sensitivity of the new detectors.

Secondly, with the speed and the availability of computational power, including cloud computing, providing tools to reduce the labor costs of Monte Carlo analysis could have a significant impact on the use of Monte Carlo analysis. With the speed of current machines and the performance of zone fire models like CFAST, it is practical to generate tens of thousands of cases to analyze. Tools that make it easier to specify tens of thousands of cases to be run and that make it easier to search, collect and process the data from those runs are needed to make the labor costs of a Monte Carlo analysis practical.

Thirdly, there is a need for a lot more data, including but not limited to fire data. For residential fires there is a need for information about the distribution of floor plans and about contents and arrangements of the contents. Importantly, the data needs to be developed in the forms of distributions. As part of the lack of data availability, there needs to be guidance about how to properly develop distributions that represent the hazards that are faced.

The final conclusion is specific to residential evacuations; there needs to be a lot more information, guidance, and modeling available to account for the impact of occupants' actions and interactions with fires. The concept of a Residential Safe Egress Time needs to be more fully explored and defined. The estimate that $70 \%$ of fires that occurred in 2003-2004 [40] did not have the fire department called out is evidence of the lack of understanding of occupant behavior in residential egress because it means that assuming an evacuation time does not address $70 \%$ of the fires that occur.

Going forward, future research should focus on a two-prong approach. First, tools are needed to make Monte Carlo analysis easier to do. Secondly, additional opportunities to apply Monte Carlo analysis to real research problems would help to build a base of expertise to further the use of this analysis.

\section{Acknowledgments}

We would like to thank Amy Mensch, Gabriel Taylor, and Nelson Bryner for their help in reviewing and improving this document. 


\section{References}

1. $\quad$ Evarts, B., Fire Loss in the United States in 2017. 2018, National Fire Protection Association: Quincy, MA.

2. Underwriters Laboratories, I., ANSI/UL 217-2015: Standard for Safety Smoke Alarms. 2015: Northbrook, IL.

3. Nelson, H.E. and F.W. Mowrer, Emergency Movement, in SFPE Handbook of Fire Protection Engineering, P.J. DiNenno, et al., Editors. 2002.

4. Bukowski, R.W., Evaluation of furniture-fire hazard using a hazard-assessment computer model. Fire and Materials, 1985. 9(4): p. 159-166.

5. Peacock, R.D. and R.W. Bukowski, A prototype methodology for fire hazard analysis. Fire Technology, 1990. 26: p. 15-40.

6. Clarke, R.W., et al., The Naional Fire Risk assessment Project - Finaly Report. , in Technical Report. 1990, National Fire Protection Research Foundation: Quincy MA.

7. $\quad$ Bukowski, R.W., et al., The National Fire Risk Assessment Project-Case Study 1: Upholstered Furniture in Residences, in Technical Report. 1990, National Fire Protection Research Foundation: Quincy MA.

8. Babrauskas, V. Fire hazard and upholstered furniture in Third Europeans Conference on Furniture Flammability 1992. Brussels: Interscience Communications Ltd. .

9. Levin, B.C., et al., Further Development of a Test Method for the Assessment of th Acute Inhalation Toxicity of Combustion Products. 1982, National Bureau of Standards: Gaithersburg, MD.

10. Peacock, R.D., et al., Defining flashover for fire haxard calculations. Fire Safety Journal 1999. 32: p. 331-345.

11. Peacock, R.D., et al., CFAST, Consolidated Model of Fire Growth and Smoke Transport (Version 7), Volume 1: Technical Reference Guide, in Technical Note 1889v1. 2015, National Institute of Standards and Technology: Gaithersburg, MD.

12. Thomas, P.H., Testing products and materials for their contribution to flashover in rooms. Fire and Materials, 1981. 5(3): p. 103-111.

13. McCaffrey, B.J., J.G. Quintiere, and M.F. Harkleroad, Estimating room fire temperature and the likelihood of flashover using fire test data correlations. Fire Technology, 1981. 17: p. 98-119.

14. Babrauskas, V., R.D. Peacock, and P.A. Reneke, Defining Flashover for fire hazard calculations: Part II. Fire Safety Journal, 2003. 38: p. 613-622.

15. Ohlemiller, T.J. and R.G. Gann, Estimating reduced fire risk resulting from an improved mattress flammability standard, in Technical Note 1446. 2002, National Institute of Standards and Technology Gaithersburg, MD.

16. Peacock, R.D., et al., Characteristics of fire scenarios in which sublethal effects of smoke are important. Fire Technology, 2004. 40: p. 127-147.

17. (ISO), I.O.f.S., ISO 13571, Life-threatening components of fire-Guidlines for the estimation of time available for escape using fire data.

18. Upadhyay, R.R. and O.A. Ezekoye, Treatment of design fire uncertainty using quadrature method of moments. Fire Safety Journal, 2008. 43: p. 127-139. 
19. Kong, D., et al., A monte carlo analysis of the heat release rate uncertainty on available safe egress time. . Journal of Fire Protection Engineering, 2012. 25: p. 5-29.

20. Bruns, M.C., Predicting the Effects of Barrier Fabrics on Residential Upholstered Furniture Fire Hazard, in NIST Technical Note 1920. 2017, National Institute of Standards and Technology: Gaithersburg MD.

21. Walton, W.D., P.H. Thomas, and Y. Ohmiya, Estimating Temperatures in Compartment Fires, in The SFPE Handbook of Fire Protection Engineering, M.J. Hurley, et al., Editors. 2016, Society of Fire Protection Engineers: New York, NY. p. 996-1023.

22. Notarianni, K.A., The Role of Uncertainty in Improving Fire Protection Regulation, in Engineering and Public Policy. 2000, Carnegie-Mellon University: Pittsburgh, PA. p. 269.

23. Notarianni, K.A. and G.W. Parry, Uncertainty, in The SFPE Handbook of Fire Protection Engineering, M.J. Hurley, et al., Editors. 2016, Society of Fire Protection Engineers: New York, NY.

24. SW, G. and B. DT, Identifying vulnerable populations to death and injuries from residential fires. Injury Prevention, 2017.

25. Standards, N.B.o., Design and Construction of Building Exits. . 1935 National Bureau of Standards Washington DC.

26. Babrauskas, V., Some Neglected Areas in Fire Safety Engineering. Fire Science and Technology, 2013. 32(1): p. 35-48.

27. Thompson, O.F., E.R. Galea, and L.M. Hulse, A review of the literature on human behaviour in dwelling fires. Safety Science, 2018. 109: p. 303-313.

28. Wood, P.G., The Behavior of People in Fires, in Fire Research Notes 953. 1972, Fire Research Station.

29. Bryan, J.L., Smoke as a Determinant of Human Behavior in Fire Situations (Project People), in GCR-77-94. 1977, National Bureau of Standards: Gaithersburg, MD.

30. Bryan, J.L. Cultural Variations in the Behavior of People in Fire Situations. in Third International Conference on Fire Safety 1978. The University of San Francisco

31. Bryan, J.L. and P.J. DiNenno, An Examination and Analysis of the Dynamics of the Human Behavior in the Fire Incident at the Georgian Towers on January 9, 1979, in GCR-79-187. 1979, National Bureau of Standards Washington, DC.

32. Proulx, G., Evacuation Time and Movement in Apartment Buildings. Fire Safety Journal, 1995. 24(3): p. 229-246.

33. Proulx, G., The Evacuation Timing, in The SFPE Handbook of Fire Protection Engineering, P.J. DiNenno, et al., Editors. 2002, Society of Fire Protection Engineers: Bethesda, MD.

34. Proulx, G., Occupant Response During a Residential High-Rise Fire. Fire and Materials, 1999. 23: p. 317-323.

35. Pauls, J.L., A Personal Perspective on Research, Consulting and Codes/Standards Development in Fire-related Human Behavior, 1969-1999, with an Emphsis on Space and Time Factors. Fire and Materials, 1999. 23: p. 265-272.

36. Sekizawa, A., et al., Occupants Behavior in Response to the High-rise Apartments Fire in Hiroshima City. Fire and Materials, 1999. 23(6): p. 297-303.

37. Hall, J.R., Jr., How many people can be saved from home fires if given more time to escape? Fire Technology, 2004. 40(2): p. 117-126. 
38. Bruck, D. and M. Ball. Sleep and Fire: Who is at Risk and Can the Risk be Reduced? in Fire Safety Science,Proceedings of the 8th International Symposium. 2005.

39. Miller, I., Behavior, Fire and Older People: Implications of the Demographic Growth of a Vulnerable Population., in Human Behavior in Fire. Proceedings of the 4th International Symposium. 2009, Interscience Communications. p. 345-354.

40. Greene, M.A. and C. Andres, 2004-2005 National Smaple Survey of Unreported Residential Fires. 2009, U.S. Consumer Product Safety Commission: Washington, DC.

41. Harpur, A.P., K.E. Boyce, and N.C. McConnell, An investigation into the circumstances surrounding fatal dwelling fires involving very young children. Fire Safety Journal, 2013. 61: p. 72-82.

42. Thompson, O.F. and D. Wales, A qualitative study of experiences, actions and motivations during accidental dwelling fires. Fire and Materials, 2015. 39: p. 453465.

43. Kahn, M.J., Detection Times to Fire-Related Stimuli by Sleeping Subjects, in NBS GCR-83-435. 1983, National Bureau of Standards: Washington, DC. p. 97 p.

44. Pearson, R.G. and M.G. Joost, Egress Behavior Response Times of Handicapped and Elderly Subjects to Simulated Residential Fire Situations, in NBS GCR 83-429. 1983, National Bureau of Standards. p. 48 p.

45. Duncan, C., The Effectiveness of the Domestic Smoke Signal, in Fire Engineering Research Report 99/5. 1999, University of Canterbury: Christchurch, NZ. p. 96.

46. Bruck, D., Non-awaking in children in response to a smoke detector alarm. Fire Safety Journal, 1999. 32(4): p. 369-376.

47. Hasofer, A.M. and D. Bruck, Statistical Analysis of Response to Fire Cues. Fire Safety Journal, 2004. 39(8): p. 663-688.

48. Ball, M. and D. Bruck. The Effect of Alcohol Upon Response to Fire Alarm Signals in Sleeping Young Adults in Human Behavor in Fire, Proceedings of the 3rd International Symposium. 2004. Belfast, N. Ireland: Interscience Communications.

49. Ashley, E.M., Waking Effectivveness of Emergency Alerting Devices for the Hearing Able, Hard of Hearing, and Deaf Populations, in Department of Fire Protection Engineering 2007, University of Maryland, College Park: College Park, MD. p. 135.

50. Kady, R.A. and J. Davey, The Impact of Exit Route Designs on Evacuation Time for Crawling Occupants. Journal Of Fire Safety Science, 2009. 27(5): p. 481-493.

51. Jin, T. and T. Yamada. Experimental Study of Human Behavior in Smoke Filled Corridors. in The Second International Symposium of Fire Safety Science

52. National Fire Protection Association, Building Construction and Safety Code. 2012, National Fire Protection Association: Quincy, MA.

53. Hurley, M.J. and E.R. Rosenbaum, Performance-Based Design, in The SFPE Handbook of Fire Protection Engineering, M.J. Hurley, et al., Editors. 2016, Society of Fire Protection Engineers: New York, NY.

54. Shields, T.J., K.E. Boyce, and N. McConnell, The Behaviour and Evacuation Experiences of WTC 9/11 Evacuees with Self-Designated Mobility Impairments. Fire Safety Journal, 2009. 44: p. 881-893.

55. Bukowski, R.W. and J.D. Averill. Methods for Predicting Smoke Detector Activation. in Fire Suppression and Detection Research Application Symposium. 1998. Orlando, FL: National Fire Protection Association. 
56. Milke, J.A., F.W. Mowrer, and P. Gandhi, Volume 3. Evaluation of Smoke Detector Performance, in Detection Performance Methodology. 2008: Quincy, MA. p. 73 p.

57. Cleary, T.G. and R.D. Peacock, A Statistical Model for Smoke Alarm Activation in upholstered Furniture Fires, in Suppression, Detection and Signaling Research and Applications Conference SUPDET 2017. 2017: Hyattsville, MD.

58. Cleary, T.G., Improving Smoke Alarm Performance - Justification for New Smoldering and Flaming Test Criteria, in Technical Note 1837. 2014, Natl. Inst. Stand. Technol. p. 27 pp.

59. Babrauskas, V., The Cone Calorimeter Used for Predictions of the Full-scale Burning Behavious of Upholstered Furniture. Fire and Materials, 1997. 21: p. 95 -105.

60. Bukowski, R.W., et al., Performance of Home Smoke Alarms: Analysis of the Response of Several Available Technologies in Residential Fire Settings, in NIST TN 1455-1, U.S.D.o. Commerce, Editor. 2008: Washington DC.

61. Carpenter, B., et al., Stan: A probabilistic programming language. Journal of Statistical Software, 2017. 76(1).

62. Greene, M.A. and A. C., 2004-2005 National Smaple Survey of Unreported Residential Fires. 2009, U.S. Consumer Product Safety Commission: Washington, DC.

63. Hamins, A., et al., Report of Experimental Results for the International Fire Model Benchmarking and Validation Exercise 3, in NIST Special Publication 1013-1 / NUREG/CR-6905. 2006, National Institute of Standards and Technology: Gaithersburg, MD.

64. Salley, M.H., et al., Verification and Validation - How to Determine the Accuracy of Fire Models. Fire Protection Engineering, 2007. 34: p. 34-44.

65. McGrattan, K.B., et al., Fire Dynamics Simulator Technical Reference Guide Volume 3: Validation, in NIST Special Publication 1018-3. 2018, National Institute of Standards and Technology Gaithersburg, MD.

66. McGrattan, K.B., R.D. Peacock, and G.P. Forney, The Long-Term Maintenance of CFAST. Fire Protection Engineering, 2017. 75(Q3): p. 30-36.

67. McGrattan, K.B., R.D. Peacock, and K.J. Overholt, Validation of Fire Models Applied to Nuclear Power Plants. Fire Technology, 2016. 52: p. 5-24.

68. Peacock, R.D., G.P. Forney, and P.A. Reneke, CFAST-Consolidated Fire And Smoke Transport (Version 7) Volume 3: Verification and Validation Guide, in NIST Technical Note 1889v3. 2017, National Istitute of Standards and Technology: Gaithersburg, MD.

69. McGrattan, K.B. and B. Toman, Quantifying the predictive uncertainty of complex numerical models. Metrologia, 2011. 48: p. 173-180.

70. Greene, W.H., Econometric Analysis (4 ${ }^{\text {th }}$ Edition). 1999: Prentice Hall.

71. McGrattan, K.B., et al., Fire Dynamics Simulator Technical Reference Guide Volume 1: Mathematical Model, in NIST Special Publication 1018-1. 2017, National Institute of Standards and Technology Gaithersburg, MD.

72. $\quad$ Peacock, R.D., et al., CFAST-Consolidated Fire And Smoke Transport (Version 7) Volume 1: Technical Reference Guide, in NIST Technical Note 1889v1. 2017, National Institute of Standards and Technology: Gaithersburg, MD.

73. McGrattan, K.B., R.D. Peacock, and K.J. Overholt, Validation of Fire Models Applied to Nuclear Power Plants. Fire Technology, 2016. 40(2): p. 117-126. 
74. Kuligowski, E.D., R.D. Peacock, and B.I. Hoskins, Review of Building Evacuation Models, in NIST Technical Note 1680. 2010, National Institute of Standards and Technology Gaithersburg MD.

75. Babrauskas, V., J.M. Fleming, and B. Russell, D. , RSET/ASET, a flawed concept for fire safety assessment. Fire and Materials, 2010. 34(7): p. 341-355.

76. Ahrens, M., False Alarms and Unwanted Activations, in U.S. Experience with Smoke Alarms and Other fire Detection/Alarm Equipment. 2004, National Fire Protection Association Quincy, MA.

77. Kuligowski, E.D., Predicting Human Behavior During Fires. Fire Technology, 2013. 49: p. 101-120.

78. Kuligowski, E.D., Modeling Building: An examination of the pre-evacuation period of the 2001 World Trade Center disaster Fire and Materials, 2015. 39(4): p. 285-300.

79. Kuligowski, E.D., et al., Guidance for the Model User on Representing Human Behavior in Egress Models. Fire Technology, 2017. 53: p. 649-672.

80. Babrauskas, V. and R.D. Peacock, Heat Release Rate - the Single Most Important Variable in Fire Hazard. Fire Safety Journal, 1992. 18(3): p. 255-272. 